\title{
Pedestal structure, stability and scalings in JET-ILW: the EUROfusion JET-ILW pedestal database
}

To cite this article before publication: Lorenzo Frassinetti et al 2020 Nucl. Fusion in press https://doi.org/10.1088/1741-4326/abb79e

\section{Manuscript version: Accepted Manuscript}

Accepted Manuscript is "the version of the article accepted for publication including all changes made as a result of the peer review process, and which may also include the addition to the article by IOP Publishing of a header, an article ID, a cover sheet and/or an 'Accepted

Manuscript' watermark, but excluding any other editing, typesetting or other changes made by IOP Publishing and/or its licensors"

This Accepted Manuscript is $\odot 2020$ The Author(s). Published by IOP Publishing Ltd..

During the embargo period (the 12 month period from the publication of the Version of Record of this article), the Accepted Manuscript is fully protected by copyright and cannot be reused or reposted elsewhere.

As the Version of Record of this article is going to be / has been published on a subscription basis, this Accepted Manuscript is available for reuse under a CC BY-NC-ND 3.0 licence after the 12 month embargo period.

After the embargo period, everyone is permitted to use copy and redistribute this article for non-commercial purposes only, provided that they adhere to all the terms of the licence https://creativecommons.org/licences/by-nc-nd/3.0

Although reasonable endeavours have been taken to obtain all necessary permissions from third parties to include their copyrighted content within this article, their full citation and copyright line may not be present in this Accepted Manuscript version. Before using any content from this article, please refer to the Version of Record on IOPscience once published for full citation and copyright details, as permissions will likely be required. All third party content is fully copyright protected, unless specifically stated otherwise in the figure caption in the Version of Record.

View the article online for updates and enhancements. 


\section{Pedestal structure, stability and scalings in JET-ILW: the EUROfusion JET-ILW pedestal database.}

L. Frassinetti ${ }^{1}$, S. Saarelma ${ }^{2}$, G. Verdoolaege ${ }^{3,4}$, M. Groth ${ }^{5}$, J.C. Hillesheim ${ }^{2}$, P. Bilkova ${ }^{6}$, P. Bohm $^{6}$, M. Dunne ${ }^{7}$, R. Fridström ${ }^{1}$, E. Giovannozzi ${ }^{8}$, F. Imbeaux ${ }^{9}$, B. Labit ${ }^{10}$, E. de la Luna ${ }^{11}$ C. Maggi ${ }^{2}$, M. Owsiak ${ }^{12}$, R. Scannell ${ }^{2}$, and JET contributors ${ }^{13^{*}}$

${ }^{1}$ Division of Fusion Plasma Physics, KTH Royal Institute of Technology, Stockholm SE

${ }^{2}$ CCFE, Culham Science Centre, Abingdon, Oxon OX14 3DB, UK

${ }^{3}$ Department of Applied Physics, Ghent University, Sint-Pietersnieuwstraat 41, 9000 Ghent, Belgium

${ }^{4}$ Laboratory for Plasma Physics-Royal Military Academy, Avenue de la Renaissancelaan 30, 1000 Brussels, Belgium

${ }^{5}$ Aalto University, Association EURATOM-Tekes, Espoo, Finland

${ }^{6}$ Institute of Plasma Physics of the CAS, Za Slovankou 3, 18200 Prague 8, Czech Republic

${ }^{7}$ Max-Planck-Institut für Plasmaphysik, Garching, Germany

${ }^{8}$ ENEA, Fusion and Nuclear Safety Department, Via E. Fermi 45, 00044 Frascati, Italy

${ }^{9}$ CEA, IRFM, F-13108 Saint-Paul-lez-Durance, France

${ }^{10}$ Swiss Plasma Center (SPC), Ecole Polytechnique Fédérale de Lausanne (EPFL),

Lausanne,Switzerland

${ }^{11}$ Laboratorio Nacional de Fusion CIEMAT, Madrid, Spain

${ }^{12}$ Poznan Supercomputing and Networking Center, IChB PAS, Noskowskiego 12/14, Poznan, Poland

${ }^{13}$ EUROfusion Consortium, JET, Culham Science Centre, Abingdon, OX14 3DB, UK

* See the author list of E. Joffrin et al. 2019 Nucl. Fusion 59112021

\section{Abstract}

The EUROfusion JET-ILW pedestal database is described, with emphasis on three main issues. First, the technical aspects are introduced, including a description of the data selection, the datasets, the diagnostics used, the experimental and theoretical methods implemented and the main definitions. Second, the JET-ILW pedestal structure and stability are described. In particular, the work describes the links between the engineering parameters (power, gas and divertor configuration) and the disagreement with the peeling-ballooning (PB) model implemented with ideal MHD equations. Specifically, the work clarifies why the JET-ILW pedestal tends to be far from the PB boundary at high gas and high power, showing that a universal threshold in power and gas cannot be found but that the relative shift (the distance between the position of the pedestal density and of the pedestal temperature) plays a key role. These links are then used to achieve an empirical explanation of the behavior of the JET-ILW pedestal pressure with gas, power and divertor configuration. Third, the pedestal database is used to revise the scaling law of the pedestal stored energy. The work shows a reasonable agreement with the earlier Cordey scaling in terms of plasma current and triangularity dependence, but highlights some differences in terms of power and isotope mass dependence. 


\section{INTRODUCTION}

H-mode databases have been created since the early 1990s [1,2]. The main goal of the early versions of H-mode databases was to study the global confinement and its scaling with engineering parameters. Due to the lack of pedestal diagnostics, detailed information on the pedestal structure were not systematically available. The first pedestal databases have been developed in the early 2000s [3,4] by a joint effort of the ITPA Confinement and Pedestal Database groups. In these works, the first scaling laws of the pedestal stored energy,were derived [4]. Soon after, the two-term model for the global confinement was developed [5, 6, 7]. The model assumes that the global energy confinement $\tau_{\mathrm{E}}$ is composed by two terms, one for the core stored energy $\left(W_{\text {core }}\right)$ and one for the pedestal stored energy $\left(W_{\text {ped }}\right), \tau_{e}=$ $\left(W_{\text {core }}+W_{\text {ped }}\right) / P$ (with $P$ the loss power). The scaling of the energy confinement was determined by the scaling law of $W_{\text {core }}$ and the scaling law of $W_{\text {ped }}$. In the following years, relatively regular updates of the database were published, in some cases with the discussion of new physics terms. For example, in 2002 the effect of the plasma magnetic geometry was included [8]. The latest update was released in 2007 by Mc Donald [9] where improved statistical methods applied to a database wider than that used by Cordey [7] were described. No drastic difference was observed between the Cordey and McDonald scaling laws. The pedestal scaling law that is still used nowadays belongs to this group of works and it is often cited as the "Cordey scaling" for the pedestal. The Cordey scaling is a power law that correlates the pedestal stored energy of Type I ELMy H-modes with engineering parameters:

$$
\begin{aligned}
W_{\text {ped }}= & 0.00807 I_{p}^{1.41 \pm 0.06} R^{1.37 \pm 0.13} P^{0.50 \pm 0.04} \times \\
& \times n^{-0.15 \pm 0.04} B^{032 \pm 0.08} M^{0.2} F_{q}^{1.61 \pm 0.17} k_{a}^{1.21 \pm 0.28}
\end{aligned}
$$

where $W_{\text {ped }}$ is the pedestal stored energy (MJ), $I_{p}$ is the plasma current (MA), $R$ the major radius (m), $P$ the thermal loss power (MW), $n$ the density $\left(10^{19} \mathrm{~m}^{-3}\right), B$ the toroidal magnetic field (T), $M$ the atomic mass, $k_{a}$ the elongation, $F_{q}$ the ratio between $q_{95}$ and the cylindrical safety factor $q_{c y l}\left(\right.$ with $\left.q_{c y l}=5 \kappa_{a} a^{2} B / R I\right)$.

Nowadays, the importance of these early works relies mainly in the scaling laws they have derived. In particular, the Cordey scaling is often used by the core transport modellers to set an edge boundary condition for predictive core transport analysis. Many of the present day predictions of core performance in JET-ILW, including D-T extrapolations, still rely on the Cordey scaling $[10,11,12,13,14]$.

Pedestal physics has progressed significantly since 2007 both in terms of experimental results and in terms of theoretical understanding. Several of the recent experimental results, in particular those achieved with metal walls like in the JET ITER-like wall (JET-ILW) [15, 16], 
might affect the predictions obtained from the Cordey scaling. Among them, the most relevant results are briefly summarized here:

(i) The first JET-ILW experimental campaign has shown that several plasmas were characterized by an energy confinement 10-20\% lower than the corresponding carbon wall JET (JET-C) plasmas. This was particularly evident in the low $\beta_{\mathrm{N}}$ pulses $\left(\beta_{\mathrm{N}} \lesssim 1.8\right.$, often called "baseline" scenario) and was mainly ascribed to a lower pedestal temperature [17, $18,19]$. Since the Cordey scaling was derived before the JET-ILW era, there is concern that expression (1) could systematically overestimate the JET-ILW pedestal performance.

(ii) Recent JET-ILW experiments investigating the isotope effect on confinement via a set of specific pulses with hydrogen plasmas and deuterium plasmas [20] have shown a thermal energy confinement with a relatively strong dependence on the isotope mass, $\tau_{E} \sim M^{0.4}$. This dependence is stronger than the dependence determined in the IPB98 $(y, 2)$ scaling [21] and included in the Cordey scaling, where the exponent was 0.2 . This difference might have an impact on the performance predictions for the deuterium-tritium operation planned in JET-ILW and for ITER.

(iii) The effect of the gas dosing $\left(\Gamma_{\text {gas }}\right)$ is not included in the Cordey scaling. This is because the gas dosing cannot be used as a reliable engineering parameter to estimate the neutral pressure, in particular when comparing different machines and/or plasmas with different wall materials. For example, in plasmas obtained in a carbon wall machine the majority of the effective fuelling is from the wall outgassing, while in the metal wall is from external fuelling. However, the gas dosing rate is well known to affect the pedestal performance, with an increasing $\Gamma_{\text {gas }}$ correlated to a decreasing pedestal pressure $[22,23,24,25,26,27$, 28, 29].

(iv) The divertor configuration has shown to play a role in determining the pedestal pressure, in particular in JET-ILW [26, 30, 31, 32].

(v) The seeding of low- $Z$ impurities has also shown to affect the pedestal performance, especially in metal wall machines where the increase of the seeding rate has been correlated with the pedestal height increase [17, 26, 28, 33, 34, 35, 36, 37, 38].

To date, there is a partial understanding of points (iii) and (v), albeit not in all machines and not for all scenarios. Concerning point (iii), the degradation of the pedestal performance with increasing gas dosing has been ascribed to the reduction of the pedestal stability due to an outward shift of the pedestal density position $\left(n_{e}^{p o s}\right)$, as initially shown in AUG [28], later confirmed in TCV [29] and in a subset of JET-ILW pulses [39]. Concerning point (v), the increase of the pedestal performance due to seeding of low-Z impurities has been explained 
only in AUG, where it has been ascribed to an inward shift of $n_{e}{ }^{\text {pos }}$ which led to an improvement of the pedestal stability [28]. Empirically, it was also found that the pedestal performance in AUG and Alcator C-mod were correlated with the separatrix density $\left(n_{e}{ }^{s e p}\right)[28,38]$.

The other experimental results discussed above, points (i), (ii) and (iv) still have no clear explanation. In particular, it is not clear yet why JET-ILW plasmas tend to have a lower pedestal performance than JET-C plasmas. It is likely that this is correlated to operational constraints in JET-ILW [19] that often needs to operate with high gas fuelling rate, but the underlying physical mechanism has not been identified yet.

Before discussing other two important open issues, it is worth to briefly summarize the main ELM types. Type I ELMs are characterized by having a frequency $\left(f_{E L M}\right)$ that increases with increasing heating power $[40,41,42]$ and are typically observed in fully developed H-modes, with heating power well above the L-H power threshold $\left(P_{L H}\right)$. Type II ELMs tends to be smaller than type I ELMs and have decreasing $f_{E L M}$ with increasing power. They have not been observed yet in JET-ILW and only mixed Type I/II ELMy regimes have been observed in JETC $[25,43,44]$, so they will not further discussed in this work. Finally, type III ELMs are typically observed just above $P_{L H}$ and are characterized by decreasing $f_{E L M}$ with increasing power $[40,45]$.

Other two important open issues are related to (vi) the agreement of the experimental data with the peeling-ballooning (PB) model in type I ELMy H-modes and (vii) the predictions of the pedestal structure, in particular the pedestal width and pedestal height.

(vi) The PB model $[46,47]$ is the most accepted one for the description of the pedestal MHD stability in type I ELMy H-modes. But a non-negligible subset of type I ELMy H-modes in JET-ILW does not seem to follow the PB model, at least when implemented with the ideal MHD equations. While most of the machines (including JET-C) have shown an agreement with the PB model [26, 37, 48, 49, 50, 51, 52], type I ELMs in JET-ILW are often triggered before the PB boundary is reached, when the pedestal gradient is well below the critical value. This has been discussed in several early and recent works [18, 27, 39, 53, 54]. In particular, a power scan at high gas dosing rate [27] has identified type I ELMs pulses by the increasing $f_{E L M}$ with power and has observed that the disagreement with the PB model occurs at high power. The present understanding is that a key condition to have pedestals far from the PB boundary is to have high gas dosing and high power. However, so far no clear universal threshold in power and gas dosing has been found. It is important to note that the disagreement with the PB model does not necessarily implies the nonvalidity of the model but it could highlight that additional physics is necessary to describe 
more properly the PB modes. For example, an hypothesis discussed in references [32, 55, 56] suggests that the inclusion of the diamagnetic term and of the experimental ion temperature in the PB stability calculation could reduce the critical gradient to a value consistent with the experimental results. Another hypothesis based on the experimental results discussed in this work is proposed at the end of the paper and suggests that the inclusion of resistivity in the MHD stability might need to be considered to fully describe the PB instabilities.

(vii) The prediction of the pedestal height and width is typically done with the EPED model $[57,58]$. However, JET-ILW has shown a disagreement with the pedestal width EPED predictions $[53,59]$. In turn, this implies that in these plasmas also the pedestal height and/or the pedestal gradient cannot be correctly predicted.

To contribute addressing these open questions related to pedestal physics, the consortium of the European nuclear fusion research community (EUROfusion [60]) has promoted the creation of a pedestal database. The creation of a state-of-the-art pedestal database is a major challenge. While the previous pedestal databases were aimed mainly at obtaining scaling laws of the pedestal height, the new EUROfusion pedestal database must contain reliable and systematic information of key experimental parameters, such as pedestal widths, pedestal gradients, pedestal positions, as well as systematic theoretical/results related to the corresponding PB stability. JET-ILW has been selected as testbed for this work. If successful, future plans include the extension to other European machines, including JET-C, AUG, TCV and MAST.

\subsection{The PB model and the EPED framework.}

The PB model assumes that the pedestal pressure increases till the PB modes become unstable and a type I ELM is triggered. The PB model can be used to determine the critical pressure gradient at which type I ELMs are supposed to be triggered, see section 2.3. To predicte the critical pedestal pressure height, the model needs to include information of the pedestal width. This can be done either using the experimental width, as described in section 2.3, or using further assumptions, like in the EPED framework. EPED is presently the most used model to predict the pedestal height and width [57]. The model relies on the stability of the PB modes and of the kinetic ballooning modes (KBMs). The models assumes that, soon after an ELM, the pedestal height and width grow unconstrained till the KBMs are destabilized. This stability boundary is considered a soft boundary that does not trigger any ELMs but set the pressure 
gradient. Once the KBM boundary is reached, the model assumes that the pedestal width $w_{p}$ depends only of the pedestal poloidal beta via the expression $\quad w_{p}=D\left(\beta \theta^{\text {ped }}\right)^{1 / 2}$, where $\mathrm{D}=0.076$ in the EPED1 version of the model [57]. This expression is often called KBM constraint. A more recent version, EPED1.6, allows a variation of the coefficient $D$, which is determined from shot to shot by studying the KBM boundary [58]. After the KBM boundary is reached, the pedestal height growths via the increase of the pedestal width and the pedestal gradient following the KBM constraint. The process continues till the PB stability limit is reached and an ELM is triggered.

Clear deviations from the exponent $1 / 2$ in the KBM constraint have been found in NSTX, where an exponent 1 was determined [61]. On the other hand, variations in $D$ have been experimentally observed in several machines with $D \approx 0.084$ in Alcator C-mod [50] to $D \approx 0.1$ in AUG [62] and JT-60U [63, 64, 65] and $D \approx 0.06-0.13$ TCV [29]. Specifically for JET-ILW, a large variation has been observed, with $D \approx 0.06-0.13[53,59]$. So a large part of JET-ILW pedestal width cannot be predicted with the standard EPED1 model. A detailed comparison between the JET-ILW pedestal and the EPED1 predictions is described in reference [66], where it is shown that EPED1 tends to underestimate the JET-IW pedestal width and overestimates the JET-ILW pressure gradient. However, the two effects partially compensate each other and the predicted pedestal height is roughly in agreement with the experimental pedestal height. On average, the agreement is within $20 \%$, even if larger deviations have also been observed. The present work will investigates under which conditions the predicted gradient departs from the experimental one. As discussed in section 8.3, the conditions under which the predicted width departs from the experimental one are similar to those of the gradient.

The model predicts the pedestal width and the pedestal height of the pressure for a given pedestal density. The pedestal density height is an input to the EPED model. Determing $n_{e}^{\text {ped }}$ has therefore high priority in pedestal physics. An attempt was done in reference [67], where the neutral penetration model was developed. Density transport in the pedestal was studied, for example, in AUG [68] and DIII-D [51, 69, 70] suggesting the transport mechanism might depend on the experimental conditions. Pedestal density predictions are an ongoing work, see for example [71], but are outside the scope of this work. Only in section 7.2 a scaling law for the JET-ILW pedestal density height will be presented. 


\subsection{Goals of the work.}

The paper has four main goals:

1. To describe the technical aspects of the database, including definitions of the key parameters and definitions of datasets, for future reference.

Due to the complexity of the database, it is important also to verify the reliability of the data included. This will be done by testing and extending recent results. Since some of the most recent results have been discussed only for few pulses, the extension to wider datasets has the benefit of strengthening their validity.

2. To provide a general description of the pedestal structure in JET-ILW in Type I ELMy H-modes.

3. To build a coherent picture of the JET-ILW pedestal physics in deuterium unseeded Type I ELMy plasmas, to help addressing some of the open questions discussed in the introduction.

In particular, the work will investigate the disagreement with the PB model and its links with plasma and engineering parameters. These links will then be used to shed more light on two open issues: the role in the pedestal dependence with power of (i) gas dosing and (ii) divertor configuration (see section 5.1 for details).

Recently, several parameters have been discovered to play an important role in pedestal physics, for example the position of pedestal density [28], the relative shift [72], the separatrix density [28], ๆe (the ratio between the gradient length of electron density and electron temperature) $[39,73,74,75,76]$. The work will exploit the EUROfusion pedestal database to reach a coherent empirical description of the links between these parameters, the engineering parameters and the agreement with the PB model. This description will then be used as the basis to shed light on the behavior of the pedestal pressure.

To achieve this goal, it will be necessary to discuss the behavior of most of the pulses included in the database but also to highlight the behavior of specific subsets. The data used in this work and the specific subsets are described in section 2.5.

4. To test the reliability of the Cordey scaling in a metal wall machine and to provide an updated version of the pedestal scaling law specific for JET-ILW.

Unfortunately, the links between pedestal stability, pedestal position, relative shift, separatrix density and $\eta \mathrm{e}$ with engineering parameters is very complex. Its understanding requires a detailed description of the behavior of the pedestal structure (pedestal height, width, position) 
in different experimental conditions. Therefore, the work adopts a systematic approach, in which each parameter of the pedestal structure is discussed in detail. Only from section 6.4, after these key ingredients have been described, coherent interconnections between engineering parameters and distance from the PB boundary will emerge. In the discussion, section 8 , these links will be extended to the pedestal pressure height.

\subsection{Structure of the paper.}

The paper is divided in four parts. Part (A) describes the technical aspects of the database, including methods and definitions. Part (B) describes the pedestal properties. Part (C) discusses the pedestal scaling. Part (D) is for discussion and conclusions.

The expert reader might wish to skip part (A) and start directly from part (B).

In details, the paper is organized as follows:

\section{PART (A). Database description.}

Section 2 describes the diagnostics used, the methods (including the experimental analysis and the peeling-ballooning stability analysis), the data selection criteria to populate the database and data used in this work.

Section 3 presents the definition of the main parameters included in the database.

Section 2 and section 3 address goal 1 .

\section{PART (B). Pedestal structure and stability.}

Section 4 briefly describes the ELMs types.

Section 5 is devoted to the pedestal structure. The section starts with the description of the pedestal height, where some open issues are introduced (role of gas dosing and divertor configuration on power scans). The section continues describing the behavior of separatrix density, pedestal width and pedestal position. In particular, this section describes novel JETILW empirical results that link the pedestal performance with the separatrix density and that correlate the separatrix density with the neutral pressure in the divertor. Section 5 addresses goal 2 and introduces the empirical basis to address goal 3.

Section 6 describes the experimental normalized pressure gradient and its comparison with PB stability. A key point of the section is to understand under which experimental conditions the JET-ILW pedestal of type I ELMy H-modes is not PB limited (i.e. the pre-ELM pedestal does not reach the PB boundary, according to the standard assumptions used in the modelling, see section 2.3). At the end of section 6, a coherent description of the links between pedestal 
stability, position, relative shift and separatrix density with engineering parameters is presented. The impact on the pedestal performance is discussed. The section addresses goal 3.

Due to the length of part (B), brief summaries are provided at the end of each section.

\section{PART (C). Pedestal scaling.}

Section 7 discusses scaling laws, comparing the Cordey scaling with the experimental JETILW results and providing an updated version of the pedestal scaling laws specific for JETILW. This section addresses goal 4.

\section{PART (D). Discussion and conclusions.}

Section 8 presents the discussion. The section starts by presenting all the links discussed in the work between engineering parameters and pedestal pressure. In particular, the empirical behavior of the pedestal pressure with power, gas and diyertor configuration presented in section 5.1 will be motivated. The section also briefly presents some of the effects not discussed in this work and how they might fit into the present work.

Section 9 presents the conclusion. The section highlights also the open questions and propose the key issue to be investigated in the near future.

Appendix 1 presents a brief comparison of the pedestal parameters determined using the different definitions discussed in section 3 .

Appendix 2 discusses the geometrical interpretation of the effect of the density shift on the pressure profile. 


\section{PART (A). Database description.}

\section{DIAGNOSTICS AND METHODS}

This section describes briefly the main diagnostics used in the work, the methods for extracting the pedestal parameters, the methods to study the peeling-ballooning stability and, finally, the methods implemented to select the pulses and a brief description of the database size.

\subsection{Diagnostics.}

The key diagnostic used to extract the pedestal parameters is the High Resolution Thomson Scattering (HRTS) [77] which measures the profiles of electron temperature $\left(T_{e}\right)$ and density $\left(n_{e}\right)$. Due to possible uncertainty in the absolute position of the HRTS diagnostic, the profiles are systematically radially shifted to have a specific temperature at the separatrix, $T_{e}{ }^{s e p}=100 \mathrm{eV}$. The value $100 \mathrm{eV}$ has been estimated using a two-point model for the power balance at the separatrix [78]. This value has been subsequently strengthened by EDGE2D-EIRENE simulations [79] that show that gas and power scans in unseeded JET-ILW plasmas can lead to $\mathrm{a} \approx 10 \%$ variation in the separatrix temperature, i.e. the range in $T_{e}{ }^{s e p} \approx 90-110 \mathrm{eV}$. Due to the fact that the profiles are very steep in the pedestal region, this variation leads to an uncertainty in the pedestal position lower than $\pm 0.002 \psi_{\mathrm{N}}$ (for a graphic visualization of this uncertainty, please see section 5.4, figures 16 and 18). Such an uncertainty has no significant effect on the pedestal parameters included in the database nor on the PB stability, as discussed in [39].

The HRTS profiles, once the $100 \mathrm{eV}$ shift is applied, are in very good agreement inside the separatrix with the profiles measured by other diagnostics, such as ECE and reflectometry [80]. However, ECE, reflectometer and Lithium beam are not systematically available in JET-ILW and they have been used only to cross-check the HRTS profiles in some key pulses. Unfortunately, the charge exchange diagnostics are available only for a limited number of pulses, so information on ion profiles are not included in the database. Although a difference between ion and electrons might be expected at the separatrix [32], the assumption $T_{e}=T_{i}$ is reasonable at the pedestal top due to the relatively high collisionality [52]. When charge exchange measurements were available, it has been verified that the assumption $T_{e}=T_{i}$ is satisfied within the uncertainties. 


\subsection{Experimental analysis technique and fits of the pre-ELM profiles}

To maximize the quality and the reliability of the pedestal parameters, the experimental HRTS profiles have been analyzed with the method described in [81] and here only briefly described. The pedestal parameters are extracted by fitting "composite" profiles selected in the pre-ELM phase during a stationary time interval. The pre-ELM phase has been defined as the time intervals in the range $70 \%-99 \%$ of the ELM cycle. This definition has been applied for most of the plasmas included in the database. When compound ELMs were present (type I ELM followed by a series of smaller ELMs), in few cases the pre-ELM selection has been optimized by considering a time window $\approx 2 \mathrm{~ms}$ long before the type I ELM. The selected profiles are then radially shifted to compensate for minor changes in the separatrix position from profile to profile. Then, the composite profile is fitted in real space and subsequently shifted in order to have the temperature fit with $100 \mathrm{eV}$ at the separatrix. Obviously, the same shift applied to the $T_{e}$ profile is applied to the $n_{e}$ profile, since both $T_{e}$ and $n_{e}$ are measured by the same diagnostic. Finally, the combined profiles, and the corresponding fits, are mapped on the normalized poloidal flux coordinate $\left(\psi_{\mathrm{N}}\right)$. An example is shown in figure 1. This method leads to well defined pedestals, with a radial resolution higher than that obtained from considering only a single profile, and consequently to pedestal parameters with good quality and good reliability.
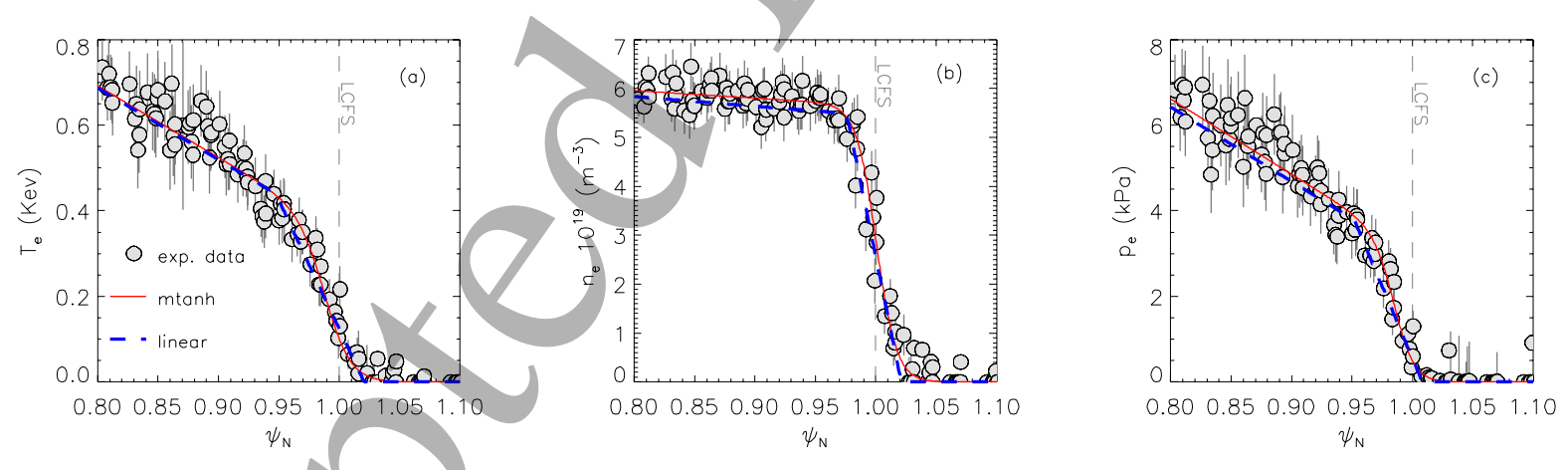

Figure 1. Example of the composite HRTS pre-ELM profiles for pulse 84600. The pre-ELM profiles have been selected in a stationary phase $1.5 \mathrm{~s}$ long, from $50.5 \mathrm{~s}$ to $52 \mathrm{~s}$. The pre-ELM profiles are defined as the profiles in the 70-99\% of the ELM cycle. In this figure, the composite profile is composed by 5 pre-ELM profiles. The continuous line shows the corresponding mtanh fit and the dashed line the linear fit. 
Two different fitting functions have been used to determine the pedestal parameters. The database contains a set of pedestal parameters for each fitting function. The first fitting function is the modified hyperbolic tangent (mtanh) defined in [82]:

$$
\operatorname{mtanh}(r)=\frac{h_{1}-h_{0}}{2}\left(\frac{(1+s x) e^{x}-e^{-x}}{e^{x}+e^{-x}}+1\right)+h_{0} \quad \text { with } \quad x=\frac{p-r}{(w / 2)}
$$

where $r$ is the radial coordinate along the HRTS line of sight, $h_{l}$ and $h_{0}$ the pedestal height and the offset in the scrape-off layer (SOL), $s$ a parameter correlated to the slope inside the pedestal top, $p$ a parameter correlated to the pedestal position and $w$ the pedestal width (see section 3.1 for more details). An example of an mtanh function is shown in figure 2(a).
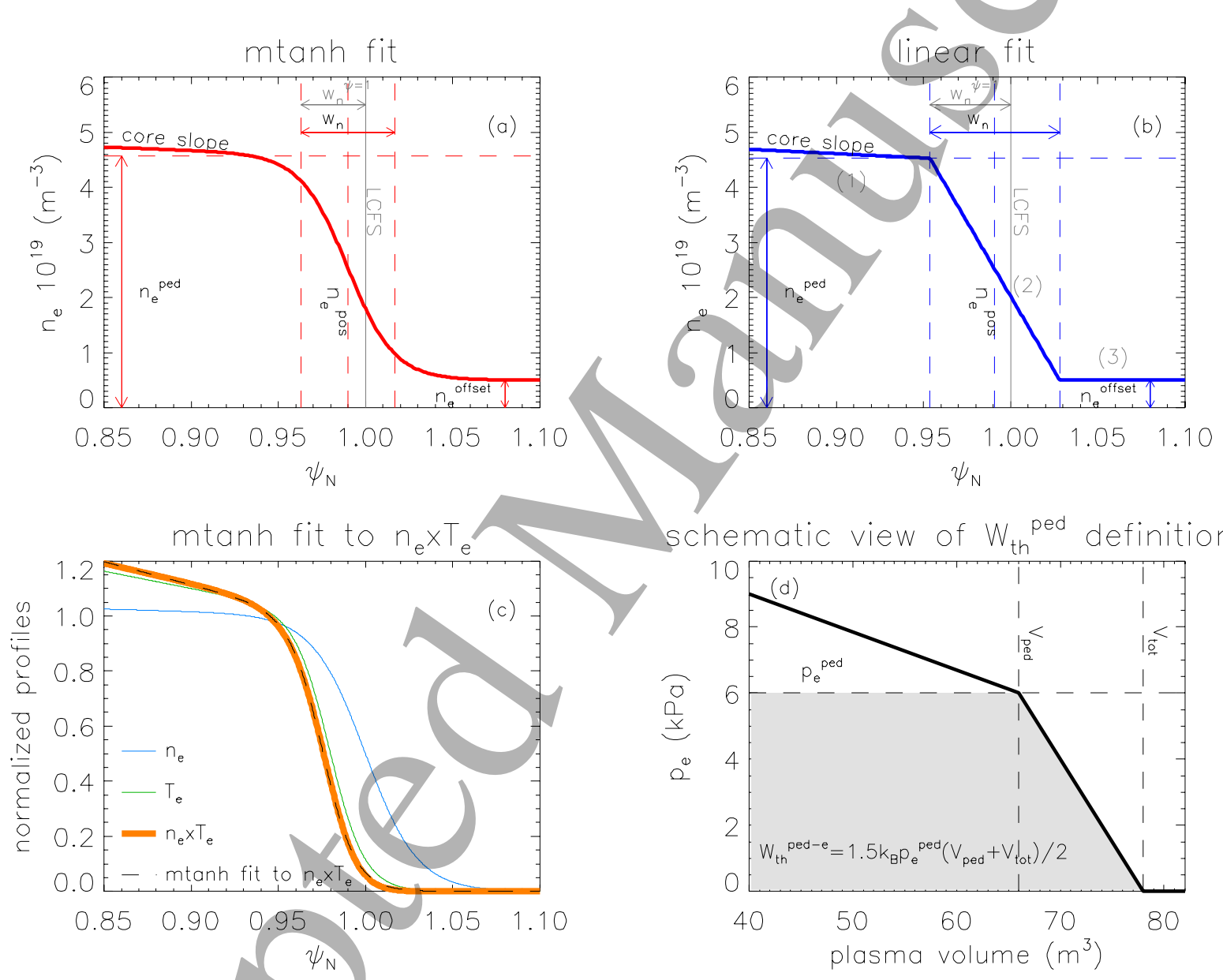

Figure 2. Example of (a) the mtanh fitting function and (b) the linear fitting functions. Frame (c) show the product of the two mtanh functions for density (blue) and temperature (green), their product (thick orange line) and the mtanh fit to the product (black dashed line). Frame (d) shows a schematic view for the calculation of the pedestal thermal stored energy, as described in section 3.2. $W_{t h}{ }^{\text {ped }}$ is the area corresponding to the region highlighted in grey (whose extent is from the magnetic axis till $V_{\text {tot, }}$ where $V_{\text {tot }}$ is the plasma volume at the separatrix), see section 3.2 for details. 
The second fitting function is a combination of linear fits, see figure 2(b) for an example. More specifically, three straight segments are used to fit (1) the inner part of the profile, inside the pedestal top, (2) the region of the steepest gradient and (3) the SOL region (where it is assumed that the slope is zero). The segments can be parameterized using the following free parameters: the position of the boundary between segments (1) and (2), the position of the boundary between segments (2) and (3), the offset in the SOL, the height at the pedestal and the slope in the inner part of the pedestal.

A comparison of the pedestal parameters extracted from the two fitting function is discussed in appendix 1. The main conclusion is that the two fitting functions lead to the same qualitative results and similar quantitative results.

\subsection{Peeling-ballooning stability analysis}

The database contains a systematic evaluation of the main key parameters related to the peeling ballooning (PB) stability. The PB model is the most accepted theoretical model for the description of the pedestal $[46,47]$. The model assumes that the pedestal evolves till the PB stability boundary is reached and a Type I ELM is triggered. Depending on several parameters, for example collisionality and triangularity, the instabilities can be either a peeling mode, a ballooning mode or a coupled peeling-ballooning modes (see reference [83] for a recent work on coupled PB modes in JET).

A standard way of representing the PB stability is to plot in the $j$ - $\alpha$ diagram the position of the experimental pedestal (the operational point) and the PB boundary. Here $j$ is the current density, composed of fully diffused Ohmic current and bootstrap current $(j b s)$, and $\alpha$ is the normalized pedestal pressure gradient defined as in [84]:

$$
\alpha=-\frac{2 \partial_{\psi} V}{(2 \pi)^{2}}\left(\frac{V}{2 \pi^{2} R}\right)^{1 / 2} \mu_{0} p^{\prime}
$$

where $V$ is the plasma volume' enclosed by the flux surface, $R$ the major radius and $p$ ' the total pressure derivative in the poloidal flux $\psi$.

In the PB stability analysis, the pressure profile $p$ is determined from the product of the mtanh fits to the pre-ELM electron density and temperature, assuming $T_{i}=T_{e}$ and determining $n_{i}$ from $n_{e}$ and $Z_{\text {eff }}$ (see section 3.2). The equilibrium is determined with HELENA [85]. HELENA is a fixed boundary equilibrium code that uses the measured density and temperature profiles for the pressure and calculates the bootstrap current profile self-consistently (in JET, the 
temperature and density profiles used by HELENA are the mtanh fits to the experimental $T_{e}$ and $n_{e}$ ). The boundary shape is taken from the standard JET EFIT equilibrium that does not have an H-mode type pressure and current profiles. While this procedure may cause a small error in the boundary shape of the plasma compared to having a true free boundary equilibrium with H-mode profiles, the effect on the shape (elongation and triangularity) is small. Therefore, we don't expect the pedestal stability to be affected. The pedestal stability with ELITE [46], a linear ideal MHD stability code. The $j_{b s}$ term has been calculated using both the Sauter model [86] and the Hager model [87]. The two models agree at low collisionality, while the Sauter model tends to overestimate $j_{b s}$ in high collisionality plasmas $[27,83,88]$, as determined by a benchmark with the drift kinetic NEO code $[89,90]$. The maximum value of the experimental normalized pressure gradient is taken from the equilibrium. The stability criterion has been defined as $\gamma<0.03 \omega_{A}$ (where $\gamma$ is the growth rate and $\omega_{\mathrm{A}}$ is the Alfvén frequency). In the stability calculation the modes from $n=5$ up to $n=70$ have been considered. The impact of higher mode numbers and of different stability criteria have been studied in reference [27], showing no major impact on the results. Rotation and diamagnetic effects have not been considered, while equal ion and electron temperature have been assumed. According to recent works, these assumptions might impact the ballooning boundary by $20-30 \%[32,55,56]$, but at present stronger effects cannot be excluded a priori (this point is discussed more in detail in section 8).

According to the PB model, the experimental pedestal in type I ELMy H-modes should reach the boundary just before the ELM is triggered. As examples, figure 3(a) shows the stability boundaries and the operational points of two type I ELMy H-modes. The operational point of pulse 81810 (red square) is clearly on the ballooning boundary (red continuous line). However, as mentioned in the introduction, the ELMs in JET-ILW are often triggered before the PB boundary is reached. This is shown in figure 3(a) with pulse 87342 (the full blue star). It is still unclear under which experimental conditions the ELM is triggered before the PB boundary is reached [39]. Both high power and high gas dosing seem a key ingredient [27]. The physical reason for this disagreement is also still unclear. Section 6 presents a systematic analysis on these aspects of the PB stability. 

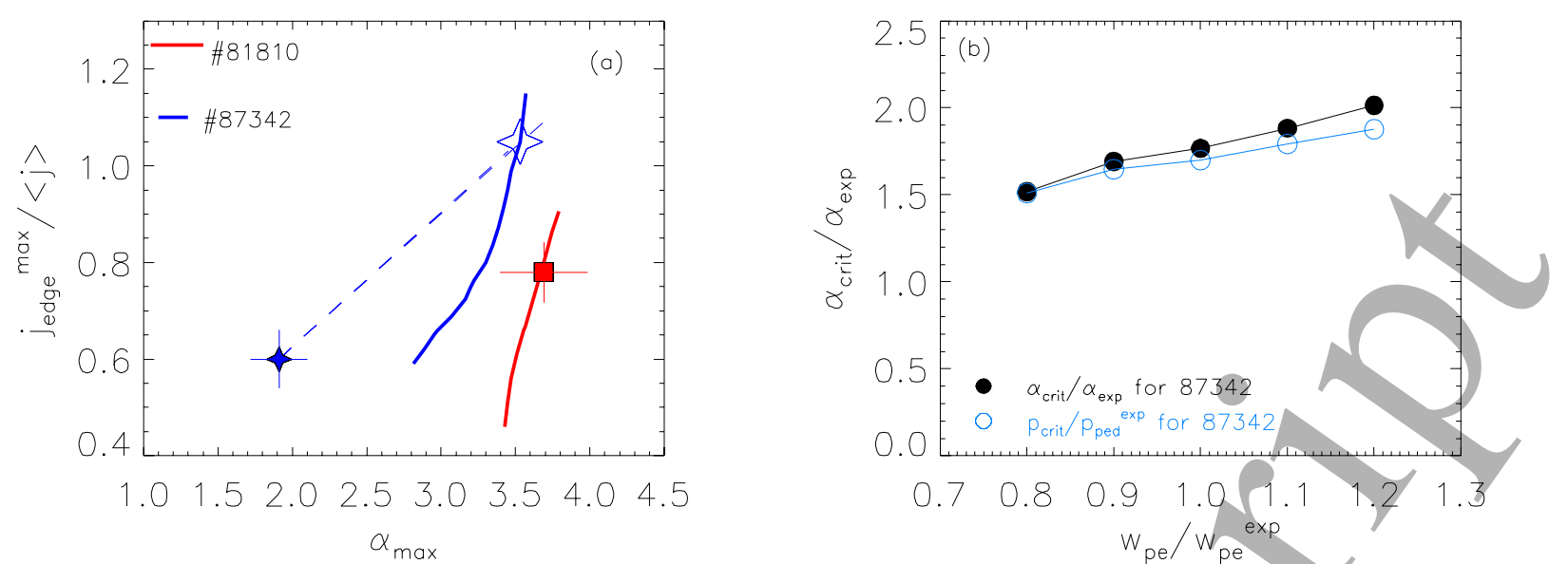

Figure 3. Frame (a). Examples of $j$ - $\alpha$ peeling-ballooning stability diagram. The $x$-axis represents the normalized pressure gradient and the $y$-axis the peak of the current density in the pedestal normalized by the average current density. The continuous lines show the PB boundaries determined with ELITE and the full symbols the operational points. The dashed line shows the self-consistent path. The intersection of the self-consistent path with the PB boundary determines $\alpha_{c r i t}$ and $j_{c r i t}$ (empty star). The square corresponds to a pre-ELM pedestal in which the ELM is triggered when the PB boundary is reached. The star shows a pre-ELM pedestal in which the ELM is triggered when the operational point is still in the stable region. Both pulses are type I ELMy H-modes. Frame (b). Sensitivity test of the dependence of $\alpha_{c r i t} / \alpha_{\text {exp }}$ on pedestal width for pulse 87342. The x-axis corresponds to the ratio between $w_{p e}$ used in the modelling and the experimental $w_{p e}$. For comparison, the blue line corresponds to the ratio between the predicted pedestal pressure and the experimental pedestal pressure.

To quantify the agreement between the experimental pre-ELM pedestal and the PB model, the approach described in reference [91] has been used. The method is based on determining the self-consistent path in the $j-\alpha$ space. The self-consistent path is determined by increasing the height of the pedestal temperature and then self-consistently calculating the current profile and the stability boundary. This is repeated till the marginally stable pedestal temperature height is reached. Graphically, the self-consistent path for pulse 87342 is represented by the dashed line of figure 3(a). The intersection of the path with the PB boundary identifies the critical normalized pressure gradient $\left(\alpha_{c r i t}\right)$, the critical current density $\left(j_{c r i t}\right)$ and the critical pedestal temperature $\left(T_{e}^{\text {crit }}\right)$. Note that $T_{e}^{\text {crit }}$ predicted with this approach differs from the EPED1 predicted temperature. The approach of the self-consistent path starts from the experimental fits and increases $T_{e}^{\text {ped }}$ till the PB boundary is reached, so the pedestal width can be considered as input parameter. The EPED1 approach assumes that the pedestal width is related only to the 
pedestal polodail beta via the expression $w_{p}=0.076\left(\beta \beta^{\text {ed }}\right)^{1 / 2}$. The ratio between $\alpha_{c r i t}$ and $\alpha_{\text {exp }}$ quantifies the agreement between experimental data and the PB model. A ratio $\alpha_{c r i t} / \alpha_{\text {exp }}>1$ implies that the ELM is triggered before the PB boundary is reached. A ratio $\alpha_{c r i t} / \alpha_{\text {exp }} \approx 1$ implies that the pedestal is PB limited. Figure 3(b) investigates if the uncertainty on the pedestal width (the pedestal parameter that has the largest uncertainty) can significantly affects the ratio $\alpha_{c r i t} / \alpha_{\text {exp }}$. This is done with the same approach of figure 3(a) but using a set of synthetic pressure profiles determined from experimental pressure profile of pulse 87342 and with different pedestal pressure width. The pedestal width has been varied by $\pm 20 \%$ which is a range wider than the typical experimental width uncertainty $(\approx \pm 10-15 \%)$. The effect of the change in the width on $\alpha_{c r i t} / \alpha_{\exp }$ is relatively mild, with $\alpha_{c r i t} / \alpha_{\exp } \approx 1.5$ when the width is $20 \%$ narrower than the experimental one and $\alpha_{c r i t} / \alpha_{\text {exp }} \approx 2.0$ when the width is $20 \%$ wider. Therefore, the uncertainty on the pedestal width cannot explain the ratio $\alpha_{c r i t}\left\langle\alpha_{\text {exp }}>>1\right.$ for pulse 87342 . Looking at the ratio of the predicted pedestal pressure to the experimental one, blue data in figure 3(b), a similar conclusion is obtained.

Hereafter, for simplicity, we will use the term "non-PB limited" when discussing the pulses for which $\alpha_{c r i t} / \alpha_{\text {exp }}>>1$. In this work, the use of the term "non-PB limited" implies that the PB stability has been determined using ideal MHD, assuming $T_{i}=T_{e}$, neglecting rotation and the diamagnetic term. The effects of these assumptions on the results presented in this work is discussed in section 8.2 .

The database includes a systematic evaluation of the parameters $T_{e}^{c r i t}, j_{c r i t}$ and $\alpha_{c r i t}$ using the method of the self-consistent path and using both Sauter and the Hager model. The two $j_{b s}$ models produce no major difference in the predicted $\alpha_{c r i t}$. This conclusion can be obtained from figure 4 , where the ratio $\alpha_{c r i t}$ hager $/ \alpha_{c r i t}$ sauter versus the pedestal collisionality is shown (for the definition of the collisionality $v^{*}$ used in this work, please see section 3.4). Most of the data included in the database have $\alpha_{c r i t}$ hager $/ \alpha_{c r i t}{ }^{\text {sauter }} \approx 1$. From a quantitative point of view, $92 \%$ of the data included in the database have $0.9<\alpha_{\text {crit }}^{\text {hager }} / \alpha_{\text {crit }}^{\text {sauter }}<1.2$. Such a difference is sufficiently small not to affect any conclusion of this work. 


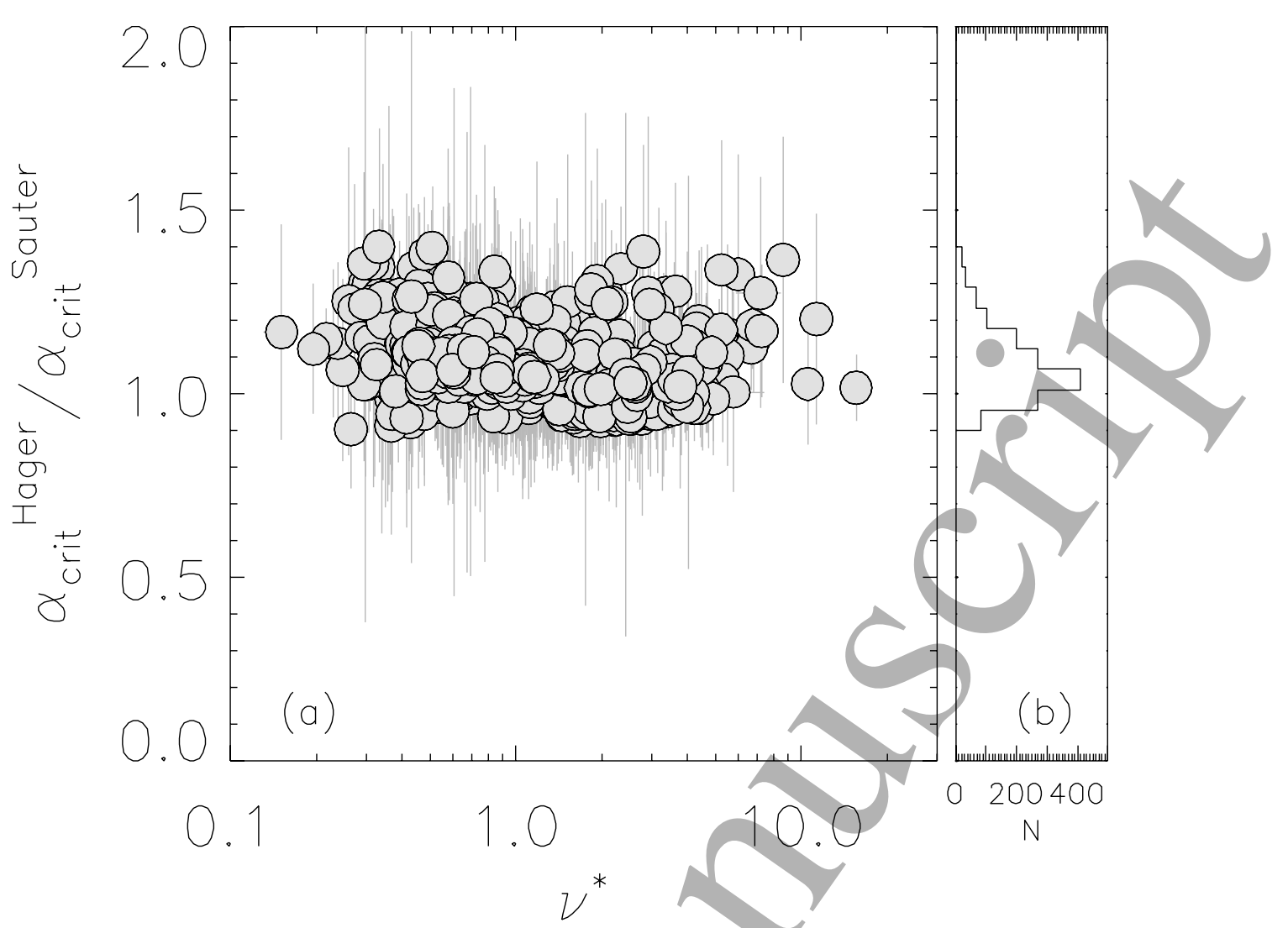

Figure 4. (a) Ratio of the critical a determined using the jbs from the Hager formula and from the Sauter formula versus the pedestal collisionality. (b) Histogram showing on the vertical axis the ratio $\alpha_{c r i t}{ }^{\text {hager }} / \alpha_{c r i t}{ }^{\text {sauter }}$ and on the horizontal axis the number of data points $(N)$ that fall within specific ranges of $\alpha_{c r i t}^{\text {hager } /} \alpha_{c r i t}$ sauter. $92 \%$ of the data are in the range $0.9<$ $\alpha_{\text {crit }}$ hager $/ \alpha_{\text {crit }}$ sauter $<1.2$. Frame (a) and frame (b) have the same range of the vertical axis.

\subsection{Selection criteria, database size, storage.}

To guarantee the reliability of pedestal parameters, the data included in the database satisfy a set of requirements.

First of all, the pre-ELM profiles are selected during a stationary phase in which the engineering parameters and the main plasma parameters $\left(\beta_{\mathrm{N}}\right.$, impurity content, line-integrated density, ELM frequency) are constant. The duration of the stationary phase $\left(\Delta t_{\text {stat }}\right)$ has been selected to be at least two energy confinement times $\left(\tau_{E}\right)$ long, $\Delta t_{s t a t} / \tau_{E}>2$ ( $\tau_{E}$ is in the range $0.1 \mathrm{~s}-0.4 \mathrm{~s}$ in JET-ILW).

The second requirement is related to the number of profiles selected to have the "composite pre-ELM profile" used for the fits (see section 2.2). To have a pedestal well resolved, the composite profile must include a minimum of two single profiles. Moreover, to maximize the 
number of selected profiles, the stationary phase must be at least $0.5 \mathrm{~s}$ long, $\Delta t_{\text {stat }}>0.5 \mathrm{~s}$. As a result, $92 \%$ of the composite profiles include more than four single profiles.

The third and last criterion assures good quality of the fits. First of all, all the experimental data and the respective fits have been visually checked in order to exclude non properly converged fits. Moreover, a quantitative criterion based on the reduced chi-square $\chi_{r}^{2}$ has been systematically implemented. The reduced chi-square is calculated as:

$$
\chi_{r}^{2}=\frac{1}{N-m} \sum_{i=0}^{N} \frac{\left(y_{i}-f\left(x_{i}\right)\right)^{2}}{\sigma_{i}^{2}}
$$

where $\left(x_{i}, y_{i}\right)$ are the $x$ - and $y$-value of the experimental data $i, \sigma_{i}$ is the corresponding uncertainty, $f\left(x_{i}\right)$ is the fit evaluated at the position $x_{i}, N-m$ is the number of degrees of freedom (with $N$ the total number of data and $m$ the number of fitted parameters). Only the data in the radial region $0.8<\psi_{\mathrm{N}}<1.05$ have been used. The model $f(x)$ properly fits the experimental data when $\chi_{r}^{2} \approx 1$. As a rule of thumb, when $\chi_{r}^{2}>2$, the model does not fit properly the data (i.e. the fit does not converge and/or the experimental data cannot be described by the model $f(x)$ ) or the experimental data are too scattered to have a reliable fit. We have set $\chi_{r}^{2}=1.5$ as the threshold to include the results in the database. This has been implemented for both the temperature data and the density data.

The database includes a total of 1121 entries. Note that a single pulse might have more than one entry, for example when a gas step or a power step is applied during the flat-top phase of the discharge. Out of the 1121 entries, 1011 corresponds to deuterium plasmas, 54 to hydrogen plasmas and 56 to mixed hydrogen/deuterium plasmas. In the deuterium group, 146 entries have a significant amount of seeding (typically nitrogen or neon, but also carbon and argon). Table 1(a) summarizes the range of the main engineering parameters for each isotope and Table $1 \mathrm{~b}$ the main global confinement parameters.

The database is stored in IMAS [92].

(a)

\begin{tabular}{cccccccc}
\hline isotope & $\mathrm{I}_{\mathrm{p}}(\mathrm{MA})$ & $\mathrm{B}_{\mathrm{t}}(\mathrm{T})$ & $\mathrm{q} 95$ & $\delta$ & $\mathrm{P}_{\mathrm{NBI}}(\mathrm{MW})$ & $\mathrm{P}_{\mathrm{ICRH}}(\mathrm{MW})$ & $\Gamma 10^{22}(\mathrm{e} / \mathrm{s})$ \\
\hline $\mathrm{D}$ & $1.0-4.0$ & $1.0-3.7$ & $2.7-4.4$ & $0.18-0.45$ & $2.5-28$ & $0.0-6.0$ & $0.07-10$ \\
$\mathrm{H}$ & $1.0-1.7$ & $1.0-1.8$ & $2.9-3.9$ & $0.20-0.35$ & $6.5-11$ & $0.0-6.0$ & $0.2-3.5$ \\
$\mathrm{H}+\mathrm{D}$ & $1.4-2.0$ & $1.6-2.3$ & $3.6-3.8$ & $0.20-0.26$ & $5.0-14$ & $0.0-3.5$ & $0.0-1.5$ \\
\hline
\end{tabular}


(b)

\begin{tabular}{cccccc}
\hline isotope & $\beta_{\mathrm{N}}$ & $\mathrm{H}_{98}$ & $\tau_{\mathrm{E}}(\mathrm{s})$ & $\mathrm{f}_{\mathrm{GW}}$ & $\mathrm{W}_{\mathrm{DIA}}(\mathrm{MJ})$ \\
\hline $\mathrm{D}$ & $0.8-3.2$ & $0.6-1.3$ & $0.1-0.4$ & $0.5-1.1$ & $1.0-10$ \\
$\mathrm{H}$ & $1.0-2.5$ & $0.7-1.1$ & $0.11-0.18$ & $0.5-0.75$ & $0.8-2.3$ \\
$\mathrm{H} / \mathrm{D}$ & $1.0-2.0$ & $0.7-1.2$ & $0.1-0.25$ & $0.45-0.8$ & $1.2-2.5$ \\
\hline
\end{tabular}

Table 1. Range of some key parameters included in the EUROfusion JET-ILW pedestal database for the deuterium dataset, hydrogen dataset and mixed hydrogen/deuterium dataset. (a) Main engineering parameters: plasma current, toroidal field, q95, average triangularity, NBI power, ICRH power, main gas dosing rate (deuterium for the D dataset and hydrogen for the $H$ dataset). (b) Main global confinement parameters: normalized beta, H98, energy confinement, Greenwald fraction, diamagnetic stored energy.

\subsection{Data used in this work and main subsets highlighted in part (B).}

The pedestal can be influenced by a large set of parameters. To simplify the investigation of the pedestal properties, this work will focus only on deuterium plasma. Moreover, pulses with seeding [17, 26, 32], with pellets (either pacing pellets or fuelling pellets) [93], with RMPs [94, 95], with kicks [96, 97] are included in the database but are excluded from the present work as these techniques can significantly affect the pedestal structure. Moreover, only type I ELMy Hmodes have been considered (see section 4). In total, the subset used in this work is composed of 767 entries.

The data contains both low triangularity $(\delta<0.3)$ and high triangularity plasmas $(\delta>0.3)$. When plotting the data of the entire database, the low $-\delta$ plasmas are highlighted with grey circles and high- $\delta$ with grey triangles. Most of the work will focus on low- $\delta$. A specific discussion for high$\delta$ plasma can be found in section 6.3.

The number of hydrogen pulses and of mixed H/D pulses available so far in JET-ILW is rather limited, so they are not discussed in this work. A description of the pedestal structure of JETILW hydrogen plasmas performed on specific subsets can be found in references [20, 98, 99]. A description of mixed H/D plasma can be found in [100]. However, due to the importance of the isotope mass in the scaling laws, section 7 is an exception and considers also the pure hydrogen pulses.

For simplicity, hereafter the term "entire database" will be used to identify all the Type I ELMy deuterium plasmas with no seeding, no pellets, no RMPs, and no kicks. 
To address the open issues introduced in goal 3 (see section 1.1), it is not sufficient to investigate generic trends observed using the entire database. As discussed in section 5, in some cases this might lead to misleading conclusions. Therefore, the work will also highlight specific subsets. Five subsets will be considered for describing the pedestal behavior with the power (three subsets at 1.4MA and two at 2.5MA):

- Power scans at 1.4MA/1.7T in low triangularity performed at

(a) low gas dosing rate $\left(\Gamma_{D 2}=0.2 \cdot 10^{22} \mathrm{e} / \mathrm{s}\right)$ in horizontal configuration (green full circles). For the definition of the configuration, see section 3.6.

(b) medium gas dosing rate $\left(\Gamma_{D 2}=0.8 \cdot 10^{22} \mathrm{e} / \mathrm{s}\right)$ in corner configuration (orange full circles).

(c) high gas dosing rate $\left(\Gamma_{D 2}=1.8 \cdot 10^{22} \mathrm{e} / \mathrm{s}\right)$ in corner configuration (light blue full circles).

For further details on these three subsets, please see references [27, 101].

- Power scans at $2.5 \mathrm{MA} / 2.4 \mathrm{~T}$ in low triangularity performed

(a) in corner configuration at gas dosing rate $\Gamma_{D 2}=(2-3) \cdot 10^{22} \mathrm{e} / \mathrm{s}$ (red empty circles) and

(b) in horizontal configuration at gas dosing rate $\Gamma_{D 2}=(1-2) \cdot 10^{22} \mathrm{e} / \mathrm{s}$ (blue empty circles). These subsets have been extracted from the database using the same criteria discussed in [30]. The subsets described above are not sufficient to reach a full description of the pedestal behavior. To describe the behavior with gas dosing and divertor configuration, three further subsets have been considered:

- Gas scan at 2.0MA/1.9-2.1T, $P_{a b s}=10 \mathrm{MW}$ and low triangularity

(a) in horizontal configuration (blue full squares),

(b) in corner configuration (red full squares),

(c) in vertical configuration (green full/squares).

JET-C is not included in the present version of the database but, for comparison with the 2MA gas scan subsets, a set of 10 JET-C pulses has been included:

- JET-C pulses at 2.0MA, no gas dosing, $P_{a b s}=10 \mathrm{MW}$, low triangularity in horizontal configuration (black squares).

These are the key subsets discussed in the papers. Color and symbol definitions have been kept consistent throughout the work.

However, to highlight some further specific behaviors, section 4, section 6.1, section 6.2 and section 6.3 will highlight also other subsets (with colors and symbols defined in order not to interfere with the subsets described above). 


\section{DEFINITIONS}

This section briefly describes the definitions of some of the key parameters included in the database. This includes both physics parameters, such as those necessary to describe the preELM pedestal structure and the pedestal performance, dimensionless parameters and ELMaveraged parameters, but also engineering parameters such as those related to the input power and the divertor configuration.

\subsection{Definitions of the pre-ELM pedestal parameters}

The pre-ELM pedestal parameters are determined by fitting the pre-ELM experimental data as described in section 2 .

\subsubsection{Definitions using the mtanh function}

Pedestal height. The pedestal height of electron density, temperature and pressure, $\left(n_{e}^{\text {ped }}, T_{e}^{\text {ped }}\right.$, $\left.p_{e}^{p e d}\right)$ are defined as the free parameter $h_{1}$ of equation (2). This definition is consistent with all previous JET analysis.

Pedestal width of temperature and density. The pedestal width of electron density and temperature ( $w_{n e}$ and $w_{T e}$ ) is represented by the parameter $w$ in equation (2). This definition is consistent with all previous JET analysis. The widths are expressed in normalized poloidal flux $\left(\psi_{\mathrm{N}}\right)$ units.

Pedestal width of pressure. Unfortunately, the determination of the width of the pedestal pressure $\left(w_{p e}\right)$ is more challenging than for $w_{T e}$ and $w_{n e}$. This is because, due to the lack of a simple deconvolution technique for the pressure, it is not possible to fit directly the experimental pressure data. In the EUROfusion pedestal database, three definitions of $w_{p e}$ have been implemented:

(i) the first definition is the standard one used in all previous JET analysis and corresponds to the average between the width of temperature and density. This is consistent with the EPED1 definition [57] and therefore it has been labeled as $w_{p e}^{E P E D}$ :

$$
w_{p e}^{E P E D}=\frac{w_{T e}+w_{n e}}{2}
$$

(ii) However, a non-negligible region of the JET-ILW pedestal density is often located outside the separatrix. See for example figure 1(b) or references $[39,72]$ or, later, figure 16(b). Typically, the PB stability analysis neglects the plasma region outside the separatrix, so, for a more direct comparison with the theoretical models, it is reasonable to define the pressure width as the region from the pedestal top till $\psi_{N}=1$. Graphically, this definition 
is shown in figure 2 and it is labeled as $w^{\psi=1}$. From a practical point of view, the temperature width is almost unaffected by this definition (this is because $T_{e}^{s e p}=100 \mathrm{eV}$, which is typically much lower than $\left.T_{e}^{p e d}\right)$. In contrast, this definition can lead to a significant reduction in the density width. As a consequence, also the corresponding pressure width, defined as

$$
w_{p e}^{\psi=1}=\frac{w_{T e}^{\psi=1}+w_{n e}^{\psi=1}}{2}
$$

tends to be lower than the $w_{p e}^{E P E D}$ definition.

(iii) The definitions above are reasonable estimates of the pressure widths but, in principle, they might lead to a biased value, for example when the position of the pedestal density and temperature are significantly different. The third definition tries to bypass this problem. Since it is not possible to fit the experimental pressure data due to the lack of a deconvolution technique, the third approach is a fit to the product of the deconvolved fits of temperature and density. An example is shown in figure 2(c), where it can be seen that the product of two mtanh function can still be fitted reasonable well by an mtanh function. This definition has been labelled simply $w_{p e}$, The corresponding uncertainty $\delta w_{p e}$ has been determined from that on $w_{T e}$ and $w_{n e}$ as $\delta w_{p e}=\sqrt{\left(\delta w_{T e}^{2}+\delta w_{n e}^{2}\right) / 2}$. A discussion on the effect of the density shift on this definition of pressure width is presented in Appendix 2.

From a quantitative point of view point, $w_{p e}$ is approximately $20-30 \%$ smaller than $w_{p e^{E P E D}}$ and up to a factor 2 larger than $w_{p e} e^{\psi=1}$. A quantitative comparison is shown in Appendix 1 and a further discussion is presented in section 5.3.

Unless otherwise stated, this work will consider only $w_{p e}{ }^{E P E D}$, for consistentcy with earlier works.

Pedestal position. The pedestal position of electron density, temperature and pressure $\left(T_{e}^{\text {pos }}\right.$, $\left.n_{e}^{p o s}, p_{e}^{p o s}\right)$ is defined as the position of the maximum gradient of the mtanh fit. This definition is consistent with the most recent JET-ILW papers [39, 72], but is slightly different from earlier works $[102,103]$, where the free parameter $p$ of equation (2) was used. The two definitions actually coincide when the core slope is negligible and, otherwise, are only slightly different. The present definition, apart from being consistent with that used in other machines, such as AUG and TCV [28, 29], is also more relevant from a physical point of view, since the position of the maximum pressure gradient is correlated to the PB stability [104]. Note that, as discussed in section 2.1, a reasonable uncertainty in $\mathrm{T}_{\mathrm{e}}{ }^{\mathrm{sep}}$ has a minimal impact on the pedestal position. The positions are expressed in normalized poloidal flux $\left(\psi_{\mathrm{N}}\right)$ units. 
Separatrix density. The separatrix electron density, $n_{e}{ }^{\text {sep }}$, is defined as the value of the $m$ tanh fit at the separatrix (after the profiles have been shifted to have $T_{e}^{s e p}=100 \mathrm{eV}$ ). The corresponding uncertainty is determined assuming a $10 \%$ error in $T_{e}^{\text {sep }}$.

Slope inside the pedestal top. The slope is correlated to the free parameter $s$ of equation (2). The parameter $s$ is actually dimensionless but a simple investigation of equation (2) shows that the core slope in physical units is $s\left(h_{1}-h_{0}\right) / w$.

SOL offset. It is defined by the free parameter $h_{0}$ in equation (2). However, the temperature profiles is very low in the SOL, so it is assumed that $h_{0}=0$ in the temperature fit. The JETILW density is also very low in the region $\psi_{\mathrm{N}}>1.05$, so it is also assumed that $h_{0}=0$ in the density fit.

\subsubsection{Definitions using the linear functions}

The free parameters used to define the linear fitting functions are already the pedestal height, offset and inner slope. The pedestal width is defined as the distance between the boundary of regions (1) and (2), and between the boundary of regions (2) and (3), see the vertical dashed lines in figure 2(b). The pedestal position is defined as the middle of region (2). As for the mtanh fit, the offsets are set to zero by default.

\subsubsection{Comparison of the definitions}

Figure 1 shows the experimental pre-ELM profiles for the pulse 84542. The dashed lines represent the mtanh fits and the continuous lines represent the linear fits. Both fitting functions describe well the experimental data. The pedestal parameters determined with the mtanh function match relatively well those from the linear fits. The two sets of parameters lead to qualitatively similar conclusions. From a quantitative point of view, a systematic comparison is shown in Appendix 1.

\subsection{Effective charge, ion density and pre-ELM pedestal thermal stored energy.}

The radial profile of the effective charge $Z_{\text {eff }}$ is not available systematically for the entire database and a line-integrated spectroscopic value has been used.

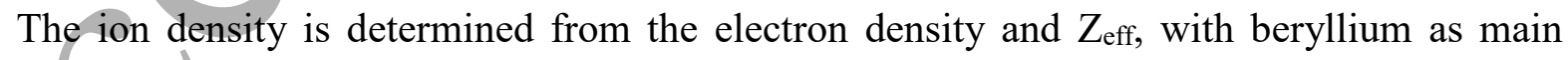
impurity in the unseeded plasmas, using the expression $n_{i}=n_{e}\left(Z_{\text {main }}+1-Z_{\text {eff }}\right) / Z_{\text {main }}$.

The pedestal thermal stored energy is defined using the same approach described in [52] and it corresponds to the plasma stored energy assuming that the pressure is not higher than $p_{e}^{\text {ped, }}$ 
neglecting all the core pressure. The expression used is the following and an explanatory schematic view can be seen in figure $2(d)$ :

$$
W_{t h}^{p e d}=\frac{3}{2} k_{B}\left(p_{e}^{p e d}+p_{i}^{p e d}\right) \frac{V_{p e d}+V_{t o t}}{2}
$$

where $k_{B}$ is the Boltzmann constant, $p_{i}^{\text {ped }}$ the ion pressure (determined assuming $T_{i}{ }^{p e d}=T_{e}{ }^{p e d}$ and calculating $n_{i}$ from $n_{e}$ and $Z_{e f f}$ ), $V_{p e d}$ is the volume at the pedestal top (defined as the volume corresponding to the postion of $p_{e}{ }^{p e d}$ ) and $V_{\text {tot }}$ the total plasma volume.

This definition is consistent with the one used by Cordey, apart from the difference that, nowadays, more detailed information of the pedestal structure is available. For example, while Cordey was obliged to introduce an ad-hoc coefficient to estimate the term $\left(V_{\text {ped }}+V_{\text {tot }}\right) / 2$, in the EUROfusion pedestal database $V_{p e d}$ is determined from the experimental data.

\subsection{Definitions of the ELM averaged pedestal heights and stored energy.}

The scaling law of the pedestal stored energy is discussed in section 7. Ideally, the definition used in this work should be consistent with that used in the earlier Cordey scaling, where ELMaveraged quantities were used. However, a perfect match of the definitions can hardly be achieved. The main reason is that, in Cordey's work, it was not possible to define precisely the pedestal height, due to the lack of diagnostics. Just as some of the possible examples, the JET$\mathrm{C}$ pedestal density used in [7] was determined with a line-integrated measurement using a chord passing near the pedestal top. For the pedestal temperature, instead, a value near the pedestal top was used (due to the lack of fully resolved profiles, it was not possible to systematically fit the experimental temperature data).

To complicate the situation, even though nowadays JET is equipped with diagnostics that resolve well the pre-ELM profiles, it remains hard to have meaningful fits of all the profiles (i.e. regardless of the position in the ELM cycle). Especially in pulses with large ELMs, the fits do not converge properly and often tend to underestimate the ELM averaged pedestal height.

For these reasons, the ELM-averaged heights included in the database have been determined without using fits. The ELM-averaged definition that has been implemented corresponds to the height at a specific radial location. All the profiles (regardless of their location in the ELM cycle) in a stationary time interval during the flat-top have been averaged at a specific radial location. The average is done for the data located in a region $0.01 \psi_{\mathrm{N}}$ wide around the chosen location. ELM-averaged heights have been determined at $\psi_{\mathrm{N}}=0.90, \psi_{\mathrm{N}}=0.91, \psi_{\mathrm{N}}=0.92, \psi_{\mathrm{N}}$ $=0.93, \psi_{\mathrm{N}}=0.94$ and $\psi_{\mathrm{N}}=0.95$. The location used for the comparison with the Cordey scaling has been decided by comparing the Cordey scaling with a small set of $30 \mathrm{JET}-\mathrm{C}$ pulses. The 
best match has been found for the ELM averaged pedestal stored energy determined at $\psi_{\mathrm{N}}=0.90$, see Appendix 1. The ELM averaged pedestal stored energy used for the scaling laws of section 7 is determined with this definition, i.e. the pedestal stored energy at $\psi_{\mathrm{N}}=0.90$, hereafter called W90.

\subsection{Dimensionless parameters.}

The dimensionless parameters are determined at the location of the pedestal top, defined as the position corresponding to $p_{e}^{\text {ped }}$. The following three dimensionless parameters are considered.

- The poloidal beta at the pedestal:

$\beta_{\theta}^{p e d}=\frac{p^{p e d}}{B_{\theta}^{2} /\left(2 \mu_{0}\right)}$

where $p^{\text {ped }}=p_{e}^{\text {ped }}+p_{i}^{\text {ped }}\left(\right.$ with $p_{i}^{\text {ped }}$ determined assuming $T_{i}^{\text {ped }}=T_{e}^{\text {ped }}$ and calculating $n_{i}$ from $n_{e}$ and $\left.Z_{e f f}\right)$ and $B \theta$ the poloidal magnetic field at the pedestal top averaged over the flux surface.

- The normalized electron-electron collisionality at the pedestal is defined as the electronelectron collision rate normalized to the thermal ion bounce frequency [86, 105]:

$v_{e}{ }^{* p e d}=6.921 \cdot 10^{-18} \ln \Lambda \frac{R q_{95} n_{e}^{\text {ped }}}{\varepsilon^{3 / 2}\left(T_{e}^{\text {ped }}\right)^{2}}$ with $\ln \Lambda=31.3-\ln \frac{\sqrt{n_{e}^{\text {ped }}}}{T_{e}^{\text {ped }}}$

where $\varepsilon=a / R$ (with $a$ and $R$ the minor and major radius respectively), the density expressed in $\mathrm{m}^{-3}$ and the temperature in $\mathrm{eV}$. The normalized electron-ion collisionality at the pedestal $v^{* p e d}$ is determined by multiplying expression (5) with the effective charge $Z_{\text {eff. }}$

- The normalized electron Larmor radius is determined as [21]:

$\rho_{e}{ }^{* p e d}=\frac{\sqrt{2 m_{e} T_{e}^{p e d}}}{\sqrt{e} B a}$

where $m_{e}$ is the electron mass in $\mathrm{kg}, e$ is the electron charge in $C, B$ the total magnetic field on axis in $T$, a the minor radius in $\mathrm{m}$ and $T_{e}^{\text {ped }}$ in $\mathrm{eV}$.

\subsection{Absorbed power, loss power and power through the separatrix.}

The total absorbed power $P_{a b s}$ has been determined as $P_{a b s}=P_{\Omega}+P_{N B I}+P_{I C R H}$, where $P_{\Omega}$ is the Ohmic power, $P_{N B I}$ is the absorbed neutral beam power, and $P_{I C R H}$ is the absorbed ion cyclotron heating power. The loss power is determined as $P=P_{a b s}-d W / d t$, where $W$ is the total stored energy. For simplicity, since only stationary time intervals have been used, the term $d W / d t$ has 
been neglected. The power through the separatrix has been determined as $P_{\text {sep }}=P_{-} P_{\text {rad }}{ }^{\text {bulk }}$, where $P_{\text {rad }}{ }^{\text {bulk }}$ is the total radiation emitted inside the separatrix.

$P_{\text {sep }}$ has been used only in section 4, when the ELMs type is discussed. The loss power $P$ has been used in section 7, when the pedestal scaling is discussed. In the rest of the work, the absorbed power $P_{a b s}$ has been used.

\subsection{Divertor configuration.}

The divertor configuration plays an important role in the pedestal performance of JET-ILW, as shown in references $[26,30,31,32]$. To help investigating the role of the divertor configuration, the database contains the coordinates of both the inner and the outer strike point as well as a simple flag with an acronym (the same nomenclature described in [30] is used and shown in figure 5). Figure 5 shows an example of the main four divertor configurations used in JETILW. The inner strike point (ISP) can be located either on the vertical target (V) or in the corner (C), while the outer strike point (OSP) can be located either on the vertical target (V), in the corner (C) or on the horizontal target. So, the acronym "V/H" stands for a divertor configuration with the ISP on the vertical target and the OSP on the horizontal target.

For simplicity, this work will consider only the OSP, therefore having only three types of divertor configuration: horizontal, corner, vertical.

Note that the vacuum pump duct is located near tile 6 , see figure $5[106,107]$. Therefore, as discussed in section 5.2, the corner configuration, is characterized by better pumping. The only available neutral pressure measurements are from the sub-divertor region. The arrow in figure 5 shows the path from the outer strike point towards the sub-divertor pressure gauge. For a clear image of the pressure gauge location, please see reference [108].

Figure 5. Examples of the four divertor configurations used in JET-ILW. The continuous lines show the inner and outer legs. The grey symbols highlight the position of the strike points for the entire database entries (low triangularity shown by circles and high triangularity by triangles). 


\section{PART (B). Pedestal structure and stability}

This part of the work describes the structure of the JET-ILW pedestal, its dependence on power, gas dosing rate and divertor configuration and compares the results with the PB stability. Section 4 is a brief discussion on the ELMs type. Section 5 describes the pedestal structure (height, separatrix density, width, position) and its links with engineering parameters. This section provides basic empirical results that will be necessary to understand the behavior of the pedestal height in JET-ILW. Section 6 describes the PB stability results and compares them with the experimental result. At the end of section 6 it will be possible to reach a coherent picture that links engineering parameters with the distance from the PB boundary.

\section{ELMs type}

The ELM type has been identified using two approaches. The first approach is based on investigating the correlation between the ELM frequency $\left(f_{E L M}\right)$ and the power through the separatrix $\left(P_{\text {sep }}\right)$. The ELM frequency has been determined by considering all the ELMs in the selected stationary time interval (see section 2.4). In this approach, it is assumed that the stationary time interval is characterized by the same ELM type. This is often correct, except for the pulses where compound ELMs are present (type I/ELM followed by a series of smaller ELMs). In these cases, extra care has been devoted to select the Thomson scattering profiles located only before the Type I ELM.

In the database, for simplicity the characterization of the ELM type has been done only for type I and type III ELMs. Type II ELMs are not considered. This simplification is motivated by the fact that no pure Type II ELM regimes have been identified so far in JET.

Type I and Type III ELMs have been identified by investigating the trend in ELM frequency with increasing $P_{\text {sep }}[40,45]$. However, no obvious trend between $f_{E L M}$ and $P_{\text {sep }}$ can be observed when looking at the entire database, as shown in figure 6(a). This is because $f_{E L M}$ is affected not only by the power. To bypass the problem, the database has been divided in many smaller subsets. Each subset is characterized by plasmas with the similar gas dosing rate, plasma current, triangularity and magnetic field. Figure 6(b) shows three examples at 3MA, 2.5MA and 1.4MA. For these specific subsets, above 4MW the ELM frequency increases with increasing $P_{\text {sep, }}$, identifying the Type I ELMs. On the contrary, the ELM frequency decreases with increasing $P_{\text {sep }}$ below $4 \mathrm{MW}$, identifying the Type III ELMs. The threshold of $4 \mathrm{MW}$ in $P_{\text {sep }}$ cannot be applied to all JET-ILW scenarios but it is nonetheless consistent with the results discussed in [20]. 
In total, the wide majority of the database is characterized by Type I ELMs. Only 20 pulses with Type III ELMs have been clearly identified.

However, this approach might not fully guarantee that all type III ELMs are identified in the database. A complementary approach is to further assume that type III ELMs can be identified depending on specific thresholds in engineering and/or plasma parameters. Unfortunately, this second approach is not straightforward, as thresholds for Type III ELMs have not been unambiguously identified in JET. For example, references [17, 24] have shown that the power threshold for the transition from Type III to type I ELMs is significantly lower in JET-ILW than in JET-C [17, 24]. Moreover, while in JET-C type III ELMs have been observed for gas dosing higher than $4-5 \times 10^{22}(\mathrm{e} / \mathrm{s})$, in JET-ILW the gas dosing threshold is higher [18], approximately $6-7 \times 10^{22}(\mathrm{e} / \mathrm{s})$. In terms of pedestal density and temperature, in JET-C type III ELMs can appear at $T_{e}^{\text {ped }}<500 \mathrm{eV}$ and $n_{e}^{\text {ped }}>4 \times 10^{19}\left(\mathrm{~m}^{-3}\right)$, while in JET-ILW only at $T_{e}^{\text {ped }}$ $<200 \mathrm{eV}$ and $n_{e} e^{\text {ped }}>5 \times 10^{19}\left(\mathrm{~m}^{-3}\right)[17,18]$.

To assure that only type I ELMs have been considered in this work, the condition of increasing $f_{E L M}$ with power has been complemented with the following thresholds: $\Gamma_{D 2}<7 \times 10^{22}(\mathrm{e} / \mathrm{s})$ and $P_{a b s} / P_{L H-08}>1.1$ (where $P_{L H-08}$ is LH power threshold defined in reference [109]). The threshold $P_{a b s} / P_{L H-08}>1.1$ has been selected to avoid to classify as type III also pulses that have been clearly identified as type I $[27,101]$, see the blue and green data in figure 6(c). The blue and green data correspond respectively to the low gas and high gas power scans discussed in reference [27] and further investigated in section 5. Note that the blue data also corresponds to the type I branch of the 1.4MA/1.7MA power scan shown in figure 6(b). It is worth to mention that the application of the thresholds $\Gamma_{D 2}<7 \times 10^{22}(\mathrm{e} / \mathrm{s})$ and $P_{a b s} / P_{L H-08}>1.1$ to the database has led automatically to consider as type I only the plasmas with $T_{e}^{\text {ped }}<200 \mathrm{eV}$ and $n_{e} e^{\text {ped }}>5 \times 10^{19}\left(\mathrm{~m}^{-}\right.$ ${ }^{3}$ ). Moreover, figure 6(c) shows that plasmas with low $H_{98}$ are not necessarily characterized by type III ELMs. In fact, the blue data have $\mathrm{H}_{98} \approx 0.8$ in the entire power scan despite having type I ELMs. It is also worth to mention that the increase of the gas fuelling at constant power tends to increase the ELM frequency and to reduce the ELM energy losses. This does not necessarily imply a transition from type 1 ELMs to type III ELMs. For example, the two data points at $P_{a b s} / P_{L H-08} \approx 1.4$ from the two power scans of figure 6(c) have both type I ELMs, but $f_{E L M} \approx 40 \mathrm{~Hz}$ for the low gas case and $f_{E L M} \approx 130 \mathrm{~Hz}$ for the high gas case. A more detailed description of the ELM energy losses is outside the scope of this work.

The following results are based only on pulses characterized by type I ELMs. 

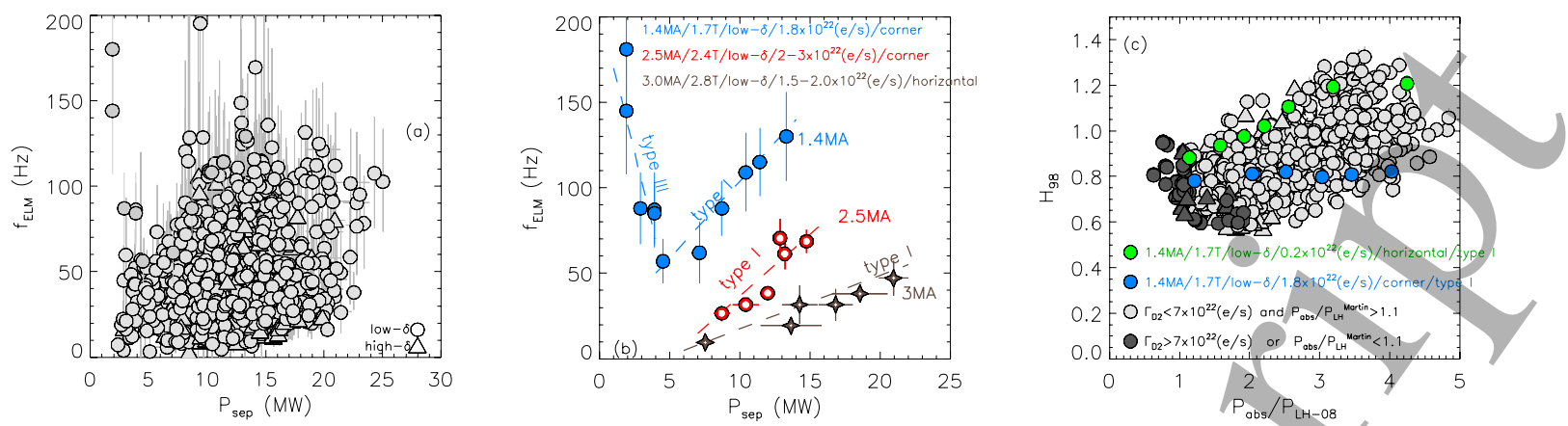

Figure 6. Frame (a) shows the ELM frequency versus the power through the separatrix for the entire dataset used in this work. Frame (b) shows the same correlation for three subsets. (i) $1.4 \mathrm{MA} / 1.7 \mathrm{~T}$, low triangularity, $\Gamma_{D 2}=1.8 \times 10^{22}(\mathrm{e} / \mathrm{s})$ is shown in light blue. The type I part of this subset corresponds to the high gas / high power scan of figure 8(d) and following figures. (ii) $2.5 \mathrm{MA} / 2.4 \mathrm{~T}$, low triangularity, $\Gamma_{D 2}=2-3 \times 10^{22}(\mathrm{e} / \mathrm{s})$ is shown in red and corresponds to the power scan in corner configuration of figure $8(\mathrm{~g})$ and following figures. (iii): 3.0MA/2.8T, low triangularity, $\Gamma_{D 2}=1.5-2.0 \times 10^{22}(\mathrm{e} / \mathrm{s})$ is shown in brown. Frame (c) shows the correlation between $H_{98}$ and the ratio between the absorbed power and the LH power threshold $P_{L H-08}$. The dark grey data highlight the subset with $P_{a b s} / P_{L H-08}<1.1$ or with gas dosing higher than $7 \times 10^{22}(\mathrm{e} / \mathrm{s})$. The blue data in frame (c) corresponds to the type I branch of the blue data of frame (b). The green data in frame (c) are similar to the blue data but have low gas dosing. The blue and the green data of frame (c) are the type I ELMY pedestals identified in reference [27] and discussed in section 5.1

\section{PEDESTAL STRUCTURE}

This section describes the pedestal structures of electron density, temperature and pressure and its dependence on the main engineering parameters. The discussion will focus on the dependence with power, gas dosing rate and divertor configuration. Unfortunately, current scans (with other engineering parameters kept constant) are not available, so only a generic discussion on the correlation with $I_{p}$ is done. Pure $q_{95}$ scans are also not available, so the correlation with $q_{95}$ is not discussed.

Section 5.1 discusses the behavior of the pedestal height. This part will provide a general overview of the pedestal height in JET-ILW and will introduce the open issues addressed in the paper (the effect of gas dosing rate and divertor configuration on power scans). Section 5.2 discusses the behavior of the separatrix density and its use as a possible proxy for the neutral pressure. This section will show that the separatrix density is an appropriate parameter to describe both the effect of gas dosing and divertor configuration on the pedestal. The pedestal width is discussed in section 5.3. The behavior of the width will be essential to understand the 
behavior of the pedestal position. Section 5.4 describes the behavior of the pedestal position and its link with the pedestal width and the separatrix density.

In all the following results, the pedestal structure has been determined using the mtanh fit. Pedestal parameters determined with the linear fit lead to the same conclusions.
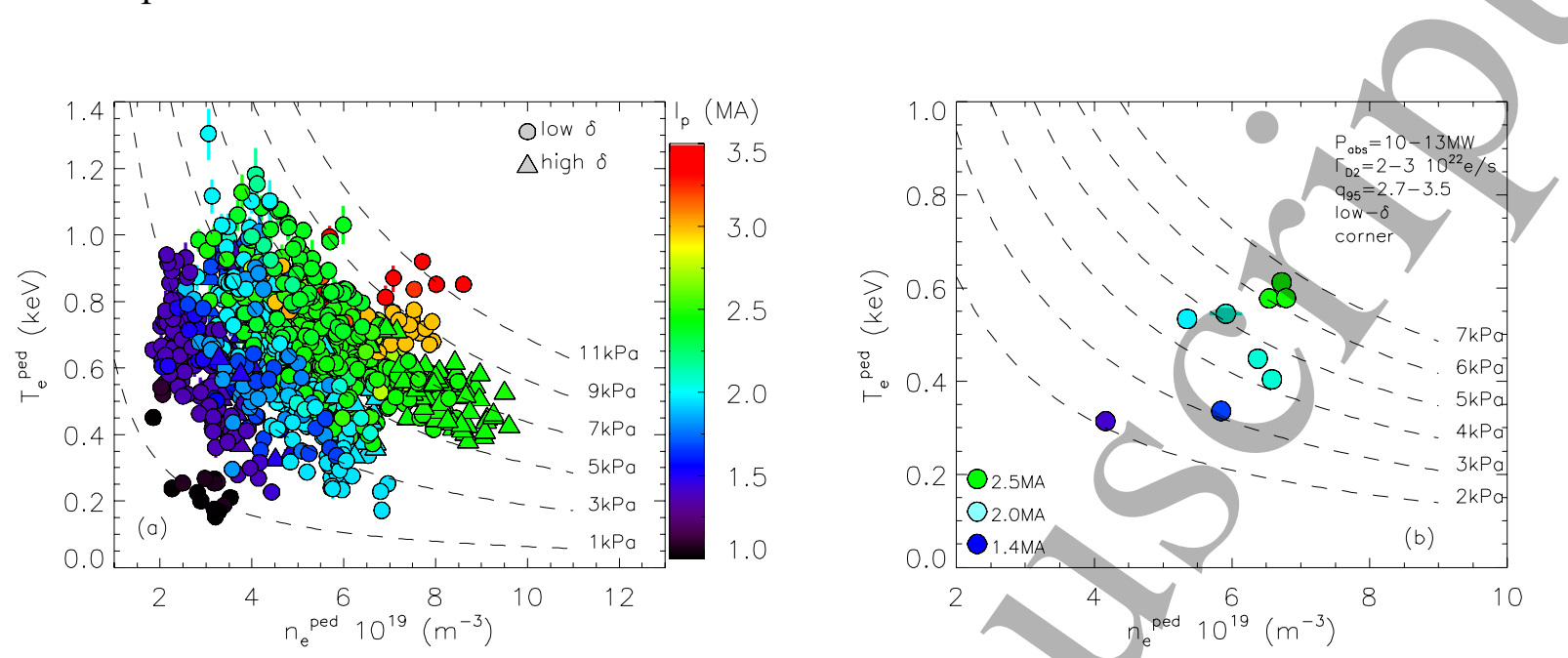

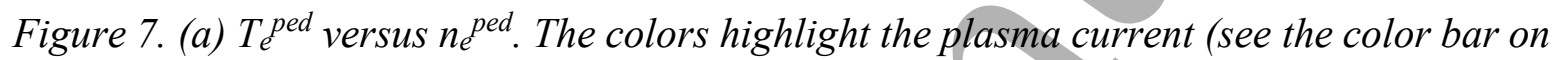
the right). The dashed lines show the isobars at constant ped. Frame (b) shows a similar diagram for a subset with different $I_{p}$, but other engineering parameters as similar as possible.

\subsection{Pedestal height}

\subsection{1 $n^{\text {ped }}-T^{\text {ped }}$ diagram and dependence on plasma current.}

As a general overview of the ranges of the pedestal height of JET-ILW, figure 7(a) shows the correlation between $T_{e}^{\text {ped }}$ and $n_{e}^{\text {ped }}$, with the colors highlighting the plasma current and the dashed lines indicating the isobars with constant $p_{e}^{\text {ped }}$.

Figure 7(a) leads to three main results:

(i) The pedestal pressure clearly increases with increasing plasma current, from $p_{e} e^{p e d}=1 \mathrm{kPa}$ at $I_{p}=1 \mathrm{MA}$ to $p_{e}{ }^{p e d}=11 \mathrm{kPa}$ at $I_{p}=3.5 \mathrm{MA}$.

(ii) The pedestal density increases with plasma current. For example, the maximum $n_{e}^{\text {ped }}$ at $1.4 \mathrm{MA}$ is $4 \cdot 10^{19} \mathrm{~m}^{-3}$, which is lower than $n_{e}^{\text {ped }}$ achieved at $2.5 \mathrm{MA}\left(8 \cdot 10^{19} \mathrm{~m}^{-3}\right)$.

(iii) High triangularity plasmas can reach higher $n_{e}^{\text {ped }}$ than low triangularity (see the green triangles $(2.5 \mathrm{MA})$ at $\left.n_{e}{ }^{\text {ped }} \approx 8-10 \cdot 10^{19} \mathrm{~m}^{-3}\right)$, as expected [43]. However, from the data of figure 5 it seems that no significant improvement is achieved by the high- $\delta$ pulses in terms of pedestal pressure. In particular, the 2.5MA data (green data) have pedestal pressure in the range $p_{e}^{\text {ped }}=5-9 \mathrm{kPa}$ for both high and low triangularity. As discussed in 
section 6 , this behavior is not a general conclusion and it is related only to the high collisionality often achieved at 2.5MA.
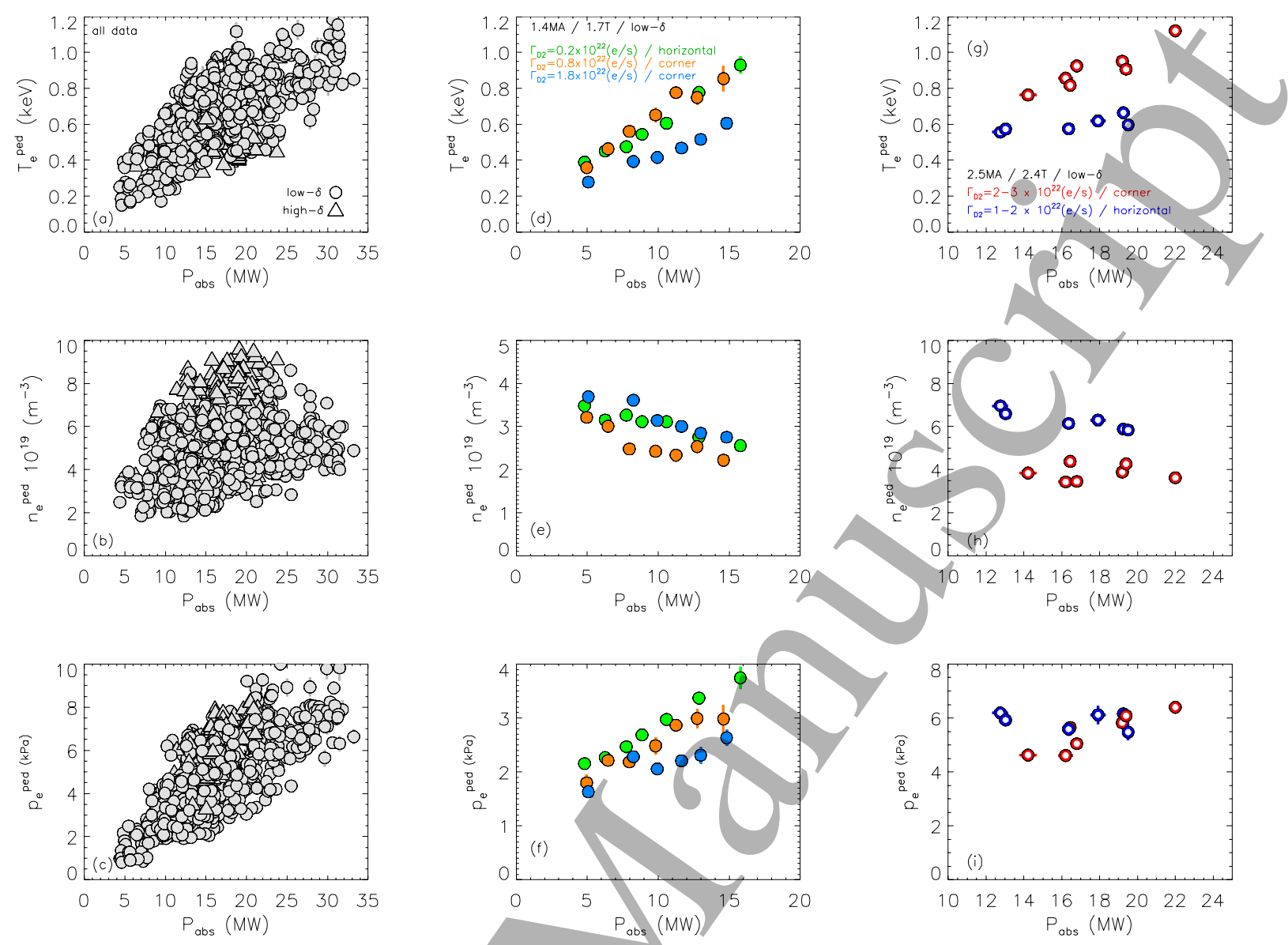

Figure 8. Correlation between the heating power absorbed by the plasma $\left(P_{a b s}\right)$ and the pedestal height of electron pedestal temperature (a), density (b) and pressure (c). The first column shows the correlation for the entire database. The second column shows the correlation for three subsets at 1.4MA/1.7T. The third column shows the correlation for two subsets at 2.5MA/2.4T.

Interestingly, figure 7(a) might seem to suggest that the maximum pedestal temperature is independent from the plasma current. The maximum $T_{e}^{\text {ped }}$ seems approximately $1.0 \mathrm{keV}$ both at low $I_{p}$ and high $I_{p}$. However, this is a misleading result and might lead to a wrong conclusion. The problem lies in the fact that at high $I_{p}$ the space of engineering parameters has not been fully covered yet in JET-ILW. In particular, high current pulses tend to have a high gas dosing rate to reduce heat loads and impurity influx (scenario development to optimize high $I_{p}$ plasmas is an ongoing area of work in JET $[110,111])$. It is very challenging to find a subset with a wide variation of current and with the other engineering parameters constant. An attempt is shown in Figure 7(b), where $I_{p}$ varies in the range 1.4MA-2.5MA. The other engineering parameters 
are as similar as possible but a perfect match cannot be achieved (for example, $q_{95}$ varies in the range 2.7-3.5). Although no conclusive claims are possible, the correlation shown in figure 7(b) suggests that increasing $I_{p}$ leads to both increasing $n_{e}^{\text {ped }}$ and increasing $T_{e}^{\text {ped }}$. This result is relevant for the discussion of the scaling laws in section 7 .

\subsubsection{Pedestal height and absorbed power.}

The dependence of the pedestal height with the absorbed heating power is shown in figure 8 On average, a clear positive correlation is observed between $P_{a b s}$ and the pedestal height of temperature and pressure, see frames (a) and (c). No clear correlation is observed between $P_{a b s}$ and the pedestal density, frame (b). However, the plasma behavior with $P_{a b s}$ is more complex than what can be concluded from the first column of figure 8 . The remaining frames of figure 8 show some specific examples.

The second column in figure 8 highlights the correlation for three subsets of pulses at 1.4MA/1.7T performed with low gas dosing rate $\left(0.2 \cdot 10^{22} \mathrm{e} / \mathrm{s}\right.$, green data), medium gas dosing rate $\left(0.8 \cdot 10^{22} \mathrm{e} / \mathrm{s}\right.$, orange data) and high gas dosing rate $\left(1.8 \cdot 10^{22} \mathrm{e} / \mathrm{s}\right.$, light blue data), see section 2.5 for details. These three examples show a qualitatively similar behavior: $T_{e}^{\text {ped }}$ and $p_{e} e^{\text {ped }}$ increase with increasing $P_{a b s}$ while $n_{e}^{\text {ped }}$ decreases. Note that the subset with the highest gas dosing rate shows a weak increase of both $T_{e}^{\text {ped }}$ and $p_{e}^{\text {ped }}$ with power [27]. Qualitatively, a positive correlation between $p_{e}{ }^{\text {ped }}$ and $P_{a b s}$ is reasonable due the expected increase of the PB stability with $\beta_{\mathrm{N}}$. From the point of view of qualitative trends, the strike point position does not seem to play a major role.

The third column in figure 8 highlights the correlation for two subsets of pulses at $2.5 \mathrm{MA} / 2.4 \mathrm{~T}$, a first subset with the outer strike point in the corner and gas dosing rate in the range $2-3 \cdot 10^{22} \mathrm{e} / \mathrm{s}$ and a second subset with outer strike point on the horizontal target and lower gas dosing rate, in the range $1-2 \cdot 10^{22} \mathrm{e} / \mathrm{s}$. The subset in the corner behaves as the 1.4MA subset, with both $T_{e}^{\text {ped }}$ and $p_{e}^{\text {ped }}$ that increase with power. Instead, the subset on the horizontal target has a much weaker increase with power, almost negligible, despite the lower gas dosing.

So, the subsets at 1.4MA and at 2.5MA highlighted in figure 8 seems to show contradicting results. This apparent contradiction is due to the fact that both gas dosing rate and the divertor configuration affect fueling and recycling, therefore the neutral pressure. Gas dosing rate alone or divertor configuration alone are not the most appropriate parameters to have a coherent description of the pedestal behavior. A more appropriate parameter might be the separatrix density, as suggested in figure 9. In fact, figure 9 shows a good correlation between the pedestal temperature and the $n_{e}{ }^{\text {sep }}$ for all the five subsets discussed in figure 8 . In particular, in figure 
9(b), the 2.5MA subset in corner configuration (red data) has lower $n_{e}{ }^{\text {sep }}$ even though the gas dosing is higher than the subset in horizontal configuration (blue subset). A possible explanation for this behavior is that the vacuum pump is located near the corner, leading to the hypothesis that the pulses in the corner configuration have better pumping, hence lower neutral pressure and lower $n_{e}{ }^{\text {sep }}$, despite the higher gas dosing rate. This hypothesis is investigated in section 5.2 .
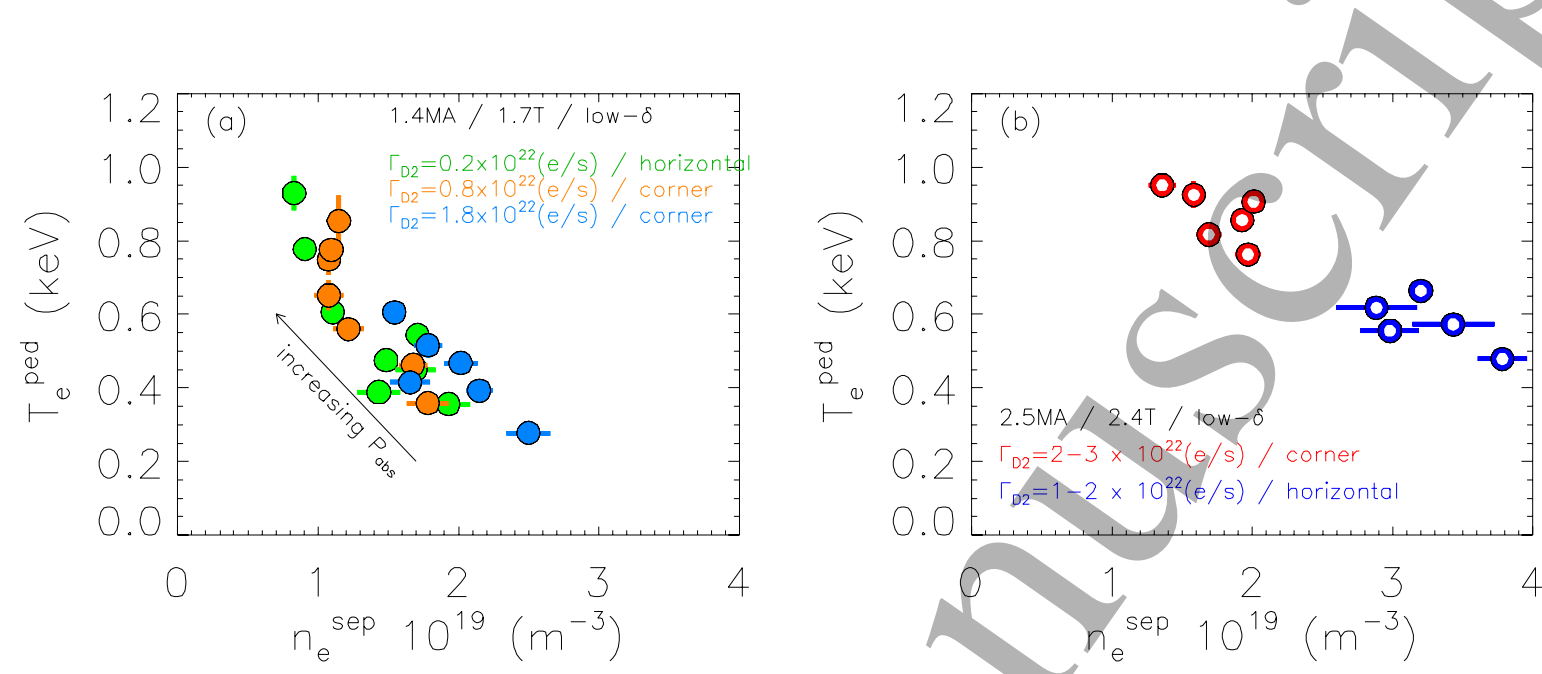

Figure 9. Correlation between pedestal temperature and separatrix density for the power scans at 1.4MA (a) and the power scans at 2.5MA (b). The same subsets of figure 8 have been used.

In conclusion, it is not possible to generalize the behavior of the pedestal height with power to only one simple single statement. The behavior with power is related to a rather complex interplay between (at least) gas dosing rate and divertor configuration. However, based on the results from figure 8 and 9 , this empirical behavior can be proposed:

- at low $n_{e}^{\text {sep }}$, both $T_{e}^{p e d}$ and $p_{e}^{\text {ped }}$ increase with increasing power, while $n_{e}^{\text {ped }}$ has a weak decrease.

- at high $n_{e}{ }^{\text {sep }}$, the increase of $T_{e}^{\text {ped }}$ and $p_{e}{ }^{\text {ped }}$ with $P_{a b s}$ is very weak and almost negligible. To understand the reason for these behaviors, first it is necessary to describe in detail the behavior of $n_{e}{ }^{\text {sep }}$, of pedestal width and their correlation with the pedestal positions. This will be done in the remaining part of this section and in sections 5.2, 5.3 and 5.4. An explanation of these behavior can be proposed only in section 6 , with details in the discussion of section 8.1 . 


\subsubsection{Dependence with gas fueling rate.}

The dependence of the pedestal height on the gas dosing rate is shown in figure 10. Considering the entire database, only the pedestal density shows a clear correlation with the dosing rate, frame (b), while pedestal temperature (a) and pressure (c) seem independent to changes in $\Gamma_{\mathrm{D} 2}$. This might seem unexpected, since the degradation of the pedestal pressure with increasing $\Gamma_{\mathrm{D} 2}$ has been observed in many machines $[23,27,28,29,39]$. The disagreement with earlier results is still present even when highlighting specific subsets characterized by the same plasma current, absorbed power, $9_{95}$ and triangularity. Clear correlations appears only when also the divertor configuration is considered. As an example, the nongrey data of figure 10 highlight a subset at $I p=2 \mathrm{MA}, P_{a b s}=10 \mathrm{MW}, q_{95}=3.0-3.3$ and low- $\delta$ (see section 2.5).

A negative trend in $T_{e}^{\text {ped }}$ and $p_{e}{ }^{\text {ped }}$ with increasing $\Gamma_{\mathrm{D} 2}$ emerges after highlighting the strike point position (see the three different colors in figure 10). Instead, the behavior of the pedestal density seems more complex, see figure 10(b). No clear correlation with the gas dosing rate is observed on the horizontal target, while a positive correlation is observed for the vertical and corner. Moreover, it is possible to note that, at the same dosing
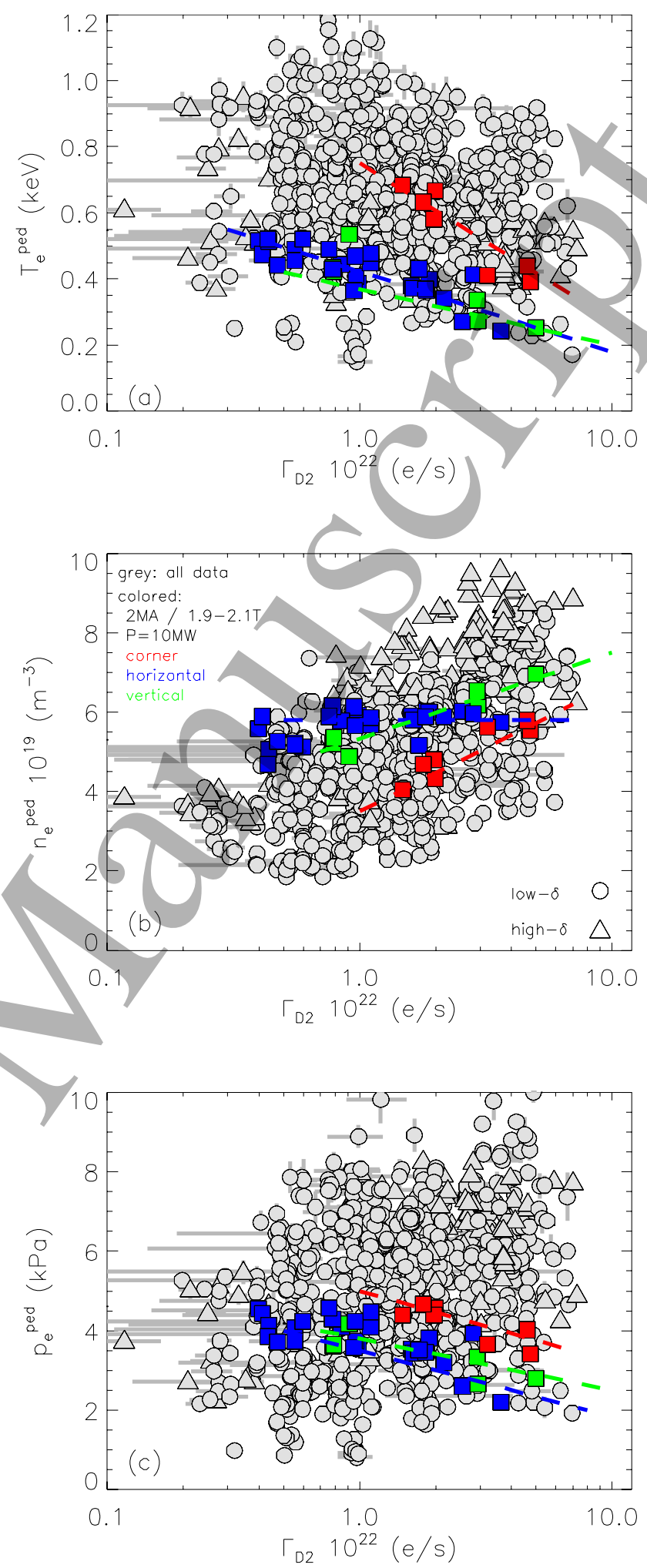

Figure 10. Correlation between the total gas fueling rate $\left(\Gamma_{D 2}\right)$ and the height of electron pedestal temperature (a), density (b) and pressure (c). The non-grey data highlight a subset at $2 M A, P_{a b s}=10 M W$, low- $\delta$ and $q_{95}=3.0$ 3.3. The different colors highlight the position of the outer strike point. 
rate, $n_{e}^{p e d}$ is up to $40 \%$ lower in the corner configuration. This observation is consistent with the discussion related to figure 9(b) and is further investigated in section 5.2.

It should be noted that the key point of this subsection is not related to the specific trend of $n_{e}{ }^{\text {ped }}$ with the gas dosing rate. Due to different plasma scenarios and/or different fueling locations, other trends might be possible. The key points are the following:

- The divertor configuration plays an important role in the pedestal performance.

- Systematic differences are observed in pulses with similar engineering parameters but different strike point position.

- The gas fueling rate is not an optimal parameter to study the pedestal performance.

As suggested in the discussion of figure 9, the key parameter is likely the neutral pressure. Unfortunately, reliable and consistent measurements of the neutral pressure in the divertor and in the main chamber are not available in JET-ILW. Based on the result of figure 9 and earlier results obtained in AUG $[28,38,112]$, a reasonable proxy to estimate the neutral pressure might be the separatrix density. This is discussed in the following section.

\subsection{Separatrix density and its correlation with the pedestal pressure}

Earlier results obtained in other machines have shown that the separatrix density is correlated with the pedestal performance. For example, in Alcator C-mod [23] a negative correlative between $n_{e}$ sep and the pedestal height was observed. A similar negative correlation has been observed in AUG in a set of gas and power scans [37]. In 2018, an AUG / Alcator C-mod comparison of $\mathrm{N}$ seeding experiments [38] has shown that the pedestal pressure height has opposite correlation with the seeding rate. On the other hand, both datasets show a clear reduction of $p^{\text {ped }}$ with increasing $n_{e}^{\text {sep }}$.

Even more recently, AUG has discussed that the separatrix density is well correlated with the divertor neutral pressure, as shown by experimental data and modelling [112,113].

This section will show with experimental results that $n_{e}{ }^{\text {sep }}$ plays an important role also in JETILW and that $n_{e}^{\text {sep }}$ is a more appropriate parameter than the fueling rate to describe the effects on the pedestal. Moreover, a simple empirical correlation will show that $n_{e}{ }^{\text {sep }}$ is a reasonably proxy for the neutral pressure also in JET-ILW. 

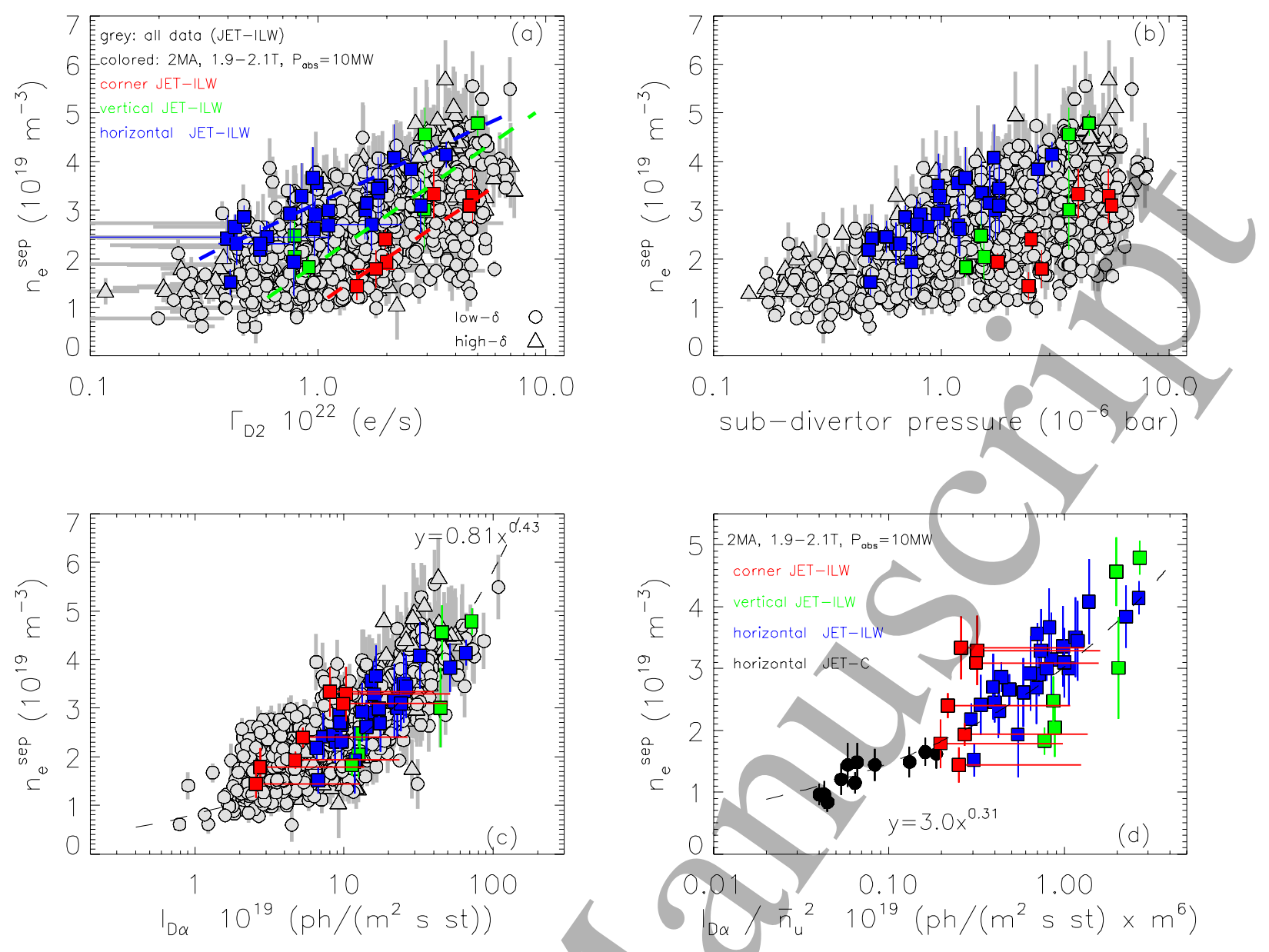

Figure 11. Correlation between $n_{e}^{\text {sep }}$ and $\Gamma_{D 2}(a)$, sub-divertor pressure (b), $I_{D \alpha}(c)$ and $I_{D \alpha} / \bar{n}_{u}^{2}(d)$. The red, blue and green data highlight the same subsets of figure 10. The error bars in the $x$-axis of the red data highlight a factor 5 increase in $I_{D \alpha}$ (data in the corner might have $I_{D \alpha}$ underestimated up to a factor 5). The black data show a set of JET-C pulses on the horizontal target and with engineering parameters similar to the JET-ILW subsets. The JET$C$ pulses have no gas dosing, so in frame (a) and (b) they are out of range. All the non-grey data are in attached conditions. The dashed lines in frames (c) and (d) shows the power law fit.

\subsection{1 $n_{e}^{\text {sep }}$ as a proxy for the neutral pressure.}

Figure 11(a) shows the correlation between $n_{e}^{\text {sep }}$ and the gas dosing rate. The scatter is rather large, but a positive correlation is present. The correlation is stronger when subsets with same engineering parameter are highlighted. The non-grey data in figure 11(a) highlights the same subsets used in figure 10 ( $I p=2 \mathrm{MA}, P_{a b s}=10 \mathrm{MW}$, low- $\delta$ and $\left.B_{t}=1.9-2.1\right)$. First of all, subsets with the same divertor configuration show a very clear correlation between $n_{e}{ }^{\text {sep }}$ and $\Gamma_{D 2}$. Second, a systematic difference between the divertor configurations is present. At the same gas dosing rate, plasmas with outer strike point in the corner have the lowest $n_{e}{ }^{\text {sep }}$, while plasmas with the outer strike point on the horizontal target tend to have the highest $n_{e}{ }^{\text {sep }}$. The hypothesis 
is that, due to the better pumping efficiency of the corner configuration directing deuterium neutrals (molecules and atoms) into the outer pumping plenum, more fuelling would be necessary to reach the same $n_{e}{ }^{\text {sep }}$ of other configurations. As discussed in references $[114,115$, 116] it is also possible that a closed divertor can trap the neutrals in a more efficient way, reducing the fuelling in the pedestal region.

To confirm this hypothesis, the best approach would be to verify the correlation between $n_{e}^{\text {sep }}$ and the neutral pressure. Unfortunately, measurements are available only in the sub-divertor region [117]. The correlation between $n_{e}{ }^{\text {sep }}$ and the sub-divertor pressure is shown in figure 11(b). Pulses with the same divertor configurations show a clear correlation between $n_{e}{ }^{\text {sep }}$ and the sub-divertor pressure. However, the systematic difference between configurations is still present. Pulses with same sub-divertor pressure have lower $n_{e}^{\text {sep }}$ in corner configuration than in horizontal or vertical configuration. The result is consistent with observations in L-mode JETILW plasmas [118, 119].

Since no direct measurement of the divertor neutral pressure is available, we have tried to use a simple approach to estimate the neutral density (hereafter, $n_{0}$ ) from the available measurements. In attached conditions, the intensity of the line emission from deuterium atoms and molecules $I_{D \alpha}$ (deuterium Balmer- $\alpha$ line) in the divertor is proportional to the density at the target $\left(n_{t}\right)$ and to the neutral density $\left(n_{0}\right)$ :

$$
I_{D \alpha} \approx n_{t} n_{0}\langle\sigma v\rangle
$$

with $n_{t}$ is related to the upstream density $\left(n_{u}\right)$ via the expression $n_{t} \propto n_{u}^{\alpha}$, where the exponent $\alpha$ is in the range $\alpha \approx 1-3$ (depending on the plasma regime being linear or high-recycling) [120]. $I_{D \alpha}$ and $n_{u}$ can be determined with the same approach used in reference [118], i.e. with a measurement spatially integrated across the outer divertor leg for $I_{D \alpha}$ and a line-averaged density (from a chord with minimum normalized radius 0.9 ) for $n_{u}$. For consistency with the $n_{e}{ }^{\text {sep }}$ data, both $I_{D \alpha}$ and $n_{u}$ have been averaged in the pre-ELM phase. The range of variation of $I_{D \alpha} / n_{u}^{\alpha}$, with $\alpha=2$, is shown in Figure 11(d). The variation in $I_{D \alpha} / n_{u}^{2}$ is larger than one order of magnitude. So, assuming that the $\langle\sigma v\rangle$ term varies much less than a factor 10, expression 7 suggests that $I_{D \alpha} / n_{u}^{2}$ might be considered as a rough estimates for the neutral density $n_{0}$ in the divertor.

The correlation between $n_{e}^{\text {sep }}$ and $I_{D \alpha}$ is shown in Figure 11(c) and the correlation between $n_{e}{ }^{\text {sep }}$ and $I_{D \alpha} / n_{u}^{\alpha}$, with $\alpha=2$, is shown in Figure 11(d). For simplicity, figure 11(d) shows only the 2MA dataset. By looking at the total ion current to the low-field-side plate versus $n_{u}$, it has been verified that all the plasmas shown in figure 11(d) are in attached conditions. 
Unfortunately, the $I_{D \alpha}$ viewing cone is strongly clipped in the corner configuration, so the data points in the corner might be strongly underestimated and can be used only to set a lower bound. The horizontal red error bars show how much a factor 5 underestimation can affect the corner dataset. However, the datasets on the horizontal and vertical targets show a very clear positive correlation between $n_{e}^{\text {sep }}$ and $I_{D \alpha} / n_{u}^{2}$, suggesting that $n_{e}^{\text {sep }}$ can be considered as a simple proxy for the neutral density.

To further strengthen this conclusion, figure 11(d) shows with black dots a set of JET-C plasmas selected with the same engineering parameters as the horizontal target JET-ILW subset. These JET-C plasmas have no gas dosing, so they cannot be shown in figure 11(a) and 11(b), due to the logarithmic scale. The fact that the JET-C pulses align very well with the JETILW subsets strengthens the hypothesis of $n_{e}{ }^{\text {sep }}$ as a proxy for $n_{0}$.

For a comparison with the AUG results discussed in reference [113], figures 11(c) and 11(d) also shows power law fits. For figure 11(c), the fit leads to the power law $n_{e}^{\text {sep }}=0.81 \times$ $\left(I_{D \alpha}\right)^{0.43}$, while for figure $11(\mathrm{~d})$ the power law is $n_{e}^{\text {sep }}=3.0 \times\left(I_{D \alpha} / n_{u}^{2}\right)^{0.31}$. This second power law has an exponent remarkably similar to what obtain in AUG using the neutral pressure $p_{0}$, where the fit $n_{e}^{\text {sep }}=2.65 \times{p_{0}}^{0.31}$ was obtained [113].

From the modelling point of view, results based on EDGE2D-EIRENE simulations applied to L-mode JET-ILW plasmas are qualitatively consistent/with this conclusion [107, 121, 122]. The extension to H-mode plasmas will be investigated in future works.
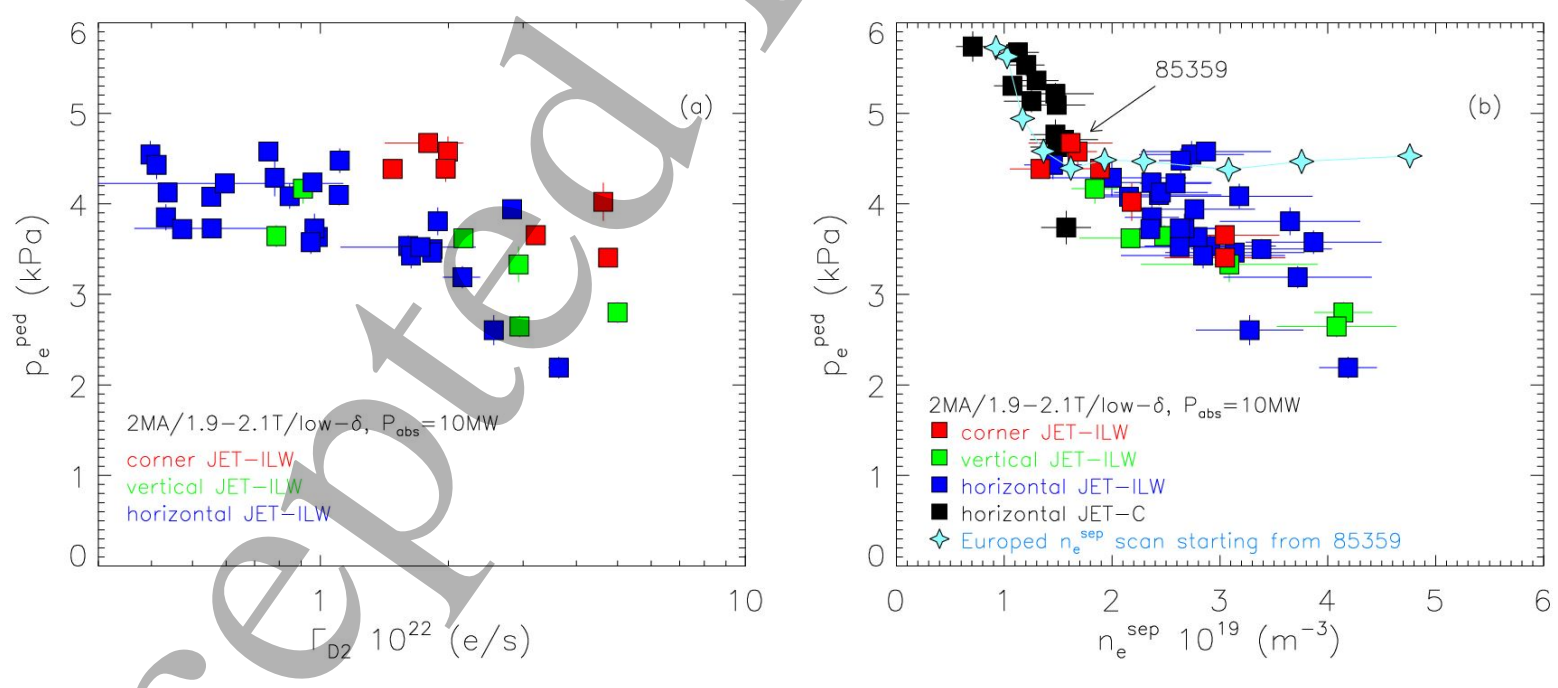

Figure 12. Correlation between $p_{e}^{\text {ped }}$ with the gas fueling rate (a) and $n_{e}{ }^{\text {sep }}$ (b). All the pulses with $p_{e} e^{p e d}<3 k P a$ are far from the PB boundary, with $\alpha_{c r i t} / \alpha_{\exp }>1.2$. The figure shows the same subsets used in figures 10 and 11. The light blue stars show the pedestal pressure predicted in a Europed $n_{e}^{\text {sep }}$ scan using pulse 85359 for the input parameters. The Europed scan in $n_{e}{ }^{\text {sep }}$ has been achieved by changing the position of the pedestal density. 


\subsection{2 $n_{e}^{\text {sep }}$ and its correlation with the pedestal pressure.}

At this stage, it is reasonable to verify if the use of $n_{e}{ }^{\text {sep }}$ instead of $\Gamma_{\mathrm{D} 2}$ removes the systematic difference shown in figure 10(c) between the divertor configurations. For simplicity, figure 12(a) shows the correlation between the pedestal pressure and $\Gamma_{\mathrm{D} 2}$ with the range of the horizontal axis optimized for the 2MA subsets. The systematic difference between divertor configurations is clear, with pulses on the corner that tend to have higher pedestal pressure than the pulses on the horizontal target. Figure 12(b) show the correlation between the pedestal pressure and $n_{e}$ sep. The systematic difference between the divertor configurations is strongly reduced. This suggests that $n_{e}{ }^{\text {sep }}$ is a parameter that includes both the effects of the fueling rate and of the divertor configuration. To reinforce this statement, figure 12(b) includes the JET-C data of figure 11(c). The JET-C data align very well with the JET-ILW subsets. Most importantly, for the same separatrix density, the pedestal heights of the JET-C and JET-ILW are similar. This suggests that the low pedestal performances of JET-ILW are, at least in part, correlated with a high separatrix density and hence with a high neutral pressure. In JET-ILW, high gas dosing that leads to high neutral pressure has been, so far, an operational necessity to achieve stable plasmas [19].

Still, a large vertical scatter remains in the data in figure 12(b). $p e^{\text {ped }}$ varies in the range 2.5$4.5 \mathrm{kPa}$ at $n_{e}{ }^{\text {sep }}=3 \times 10^{19} \mathrm{~m}^{-3}$. This can imply that (i) $n_{e}{ }^{\text {sep }}$ is not the only parameter that describes the physics of a gas scan and/or that (ii) the use of $n_{e}{ }^{\text {sep }}$ as a proxy for the neutral density is too crude. Future works will try to address this point.

It is important to highlight that the trend between $p_{e}^{\text {ped }}$ and $n_{e}^{\text {sep }}$ for the datasets of figure 12(b) is robust and other subsets at 1.4MA and at 2.5MA shows qualitatively similar correlations (see for example figure 9).

From an empirical point of view, this result is very important. In principle, it can improve the pedestal predictions in scaling laws, where the effects of different divertor configurations and of gas fueling are not considered. The inclusion in the scaling laws of $n_{e}{ }^{\text {sep }}$ can likely remove the systematic effects produced by different strike point positions and different fueling rate. This idea is tested in section 7.

From a physical point of view, the correlation of figure 12(b) is understood only in part. As discussed later, in section 5.4, the increase in $n_{e}{ }^{\text {sep }}$ is correlated with the outward shift of $n_{e}{ }^{p o s}$ $[23,28,39]$. The idea is that the increase of $n_{e}{ }^{\text {sep }}$ leads to an outward shift of the density which in turn reduces the PB stability. This hypothesis is tested with Europed in figure 12(b). Europed implements the EPED1 model using HELENA [85] for the equilibrium and ELITE [46] for the 
linear ideal MHD stability of the PB modes [123]. Initially, Europed has been run for pulse 85359 (JET-ILW pulse at 2MA, corner configuration with low $n_{e}{ }^{\text {sep }} \approx 1.5 \cdot 10^{19} \mathrm{~m}^{-3}$ ). Europed predicts a pedestal pressure consistent with the experimental result $\left(p_{e}^{\text {ped }}=4.5 \mathrm{kPa}\right.$ and $p_{e}{ }^{p e d}=4.7 \pm 0.1 \mathrm{kPa}$ respectively). Next, Europed has been run by changing systematically the position of the pedestal density. The predicted $n_{e}^{\text {sep }}$ and $p_{e}{ }^{\text {ped }}$ have been determined from the critical profiles and are shown in figure 12(b) with light blue stars. The predicted pressure shows a clear reduction with increasing separatrix density and a good quantitative agreement with the experimental data till $n_{e}^{s e p} \approx 1.5 \cdot 10^{19} \mathrm{~m}^{-3}$. Note that the predicted pedestal pressure covers the range of the JET-C pulses and the range of the highest $p_{e}{ }^{\text {ped }}$ JET-ILW pulses. This strengthens the idea discussed above that the low pedestal performances of JET-ILW are in part correlated with the high $n_{e}^{\text {sep }}$.

On the other hand, the trend of the predicted $p_{e} e^{\text {ped }}$ with $n_{e}{ }^{\text {sep }}$ saturates when $n_{e}{ }^{\text {sep }}>1.5 \cdot 10^{19} \mathrm{~m}^{-3}$. At high separatrix density, the Europed model is not able anymore to explain the experimental data. The saturation is due to the fact that the PB stability is affected by the pressure position and not by the density position. When the density positon is too far outward (i.e. at high $n_{e}{ }^{\text {sep }}$ ) the change in $n_{e}{ }^{p o s}$ does not affect $p_{e}^{p o s}$, as shown in [39] and further discussed in Appendix 2. As a consequence, no change in the PB stability can be expected. At present, the mechanism that explains the $p_{e}^{\text {ped }}$ degradation at high $n_{e}$ sep is not understood. In section 6 it will be shown that the high $n_{e}{ }^{\text {sep }}$ pulses are far from the PB boundary and some hypothesis on the degradation mechanism will be put forward.

The key messages of the subsection are the following:

- The separatrix density might be used as a simple proxy for the neutral density in JET-ILW and might be a more appropriate parameter to estimate the neutral density then the gas fueling rate.

- The use of the separatrix density seems to remove the systematic difference in $p_{e}^{\text {ped }}$ between divertor configurations.

- JET-C and JET-ILW pulses with similar engineering parameters and same $n_{e}^{\text {sep }}$ have similar pedestal pressure.

- The pedestal pressure degrades with increasing separatrix density.

- The degradation mechanism is understood only at low $n_{e}{ }^{\text {sep }}$, where it is due to the outward shift of the density. 


\subsection{Pedestal width}

A reliable estimate of the pedestal width is vital for pedestal physics. The pedestal width is essential to determine the pedestal gradient and hence the pedestal stability. A reliable estimate of the width is important also for pedestal predictions. For example, the EPED1 model [57] assumes that the pressure width scales as $w_{p e}{ }^{E P E D}=D\left(\beta_{\theta}{ }^{p e d}\right)^{1 / 2}$ with $D=0.076$, as determined from an experimental fit of DIII-D low $v^{*}$ plasmas (where $w_{p e} e^{E P E D}$ is defined as the average between the temperature and the density width, consistent with the definitions of section 3.1).
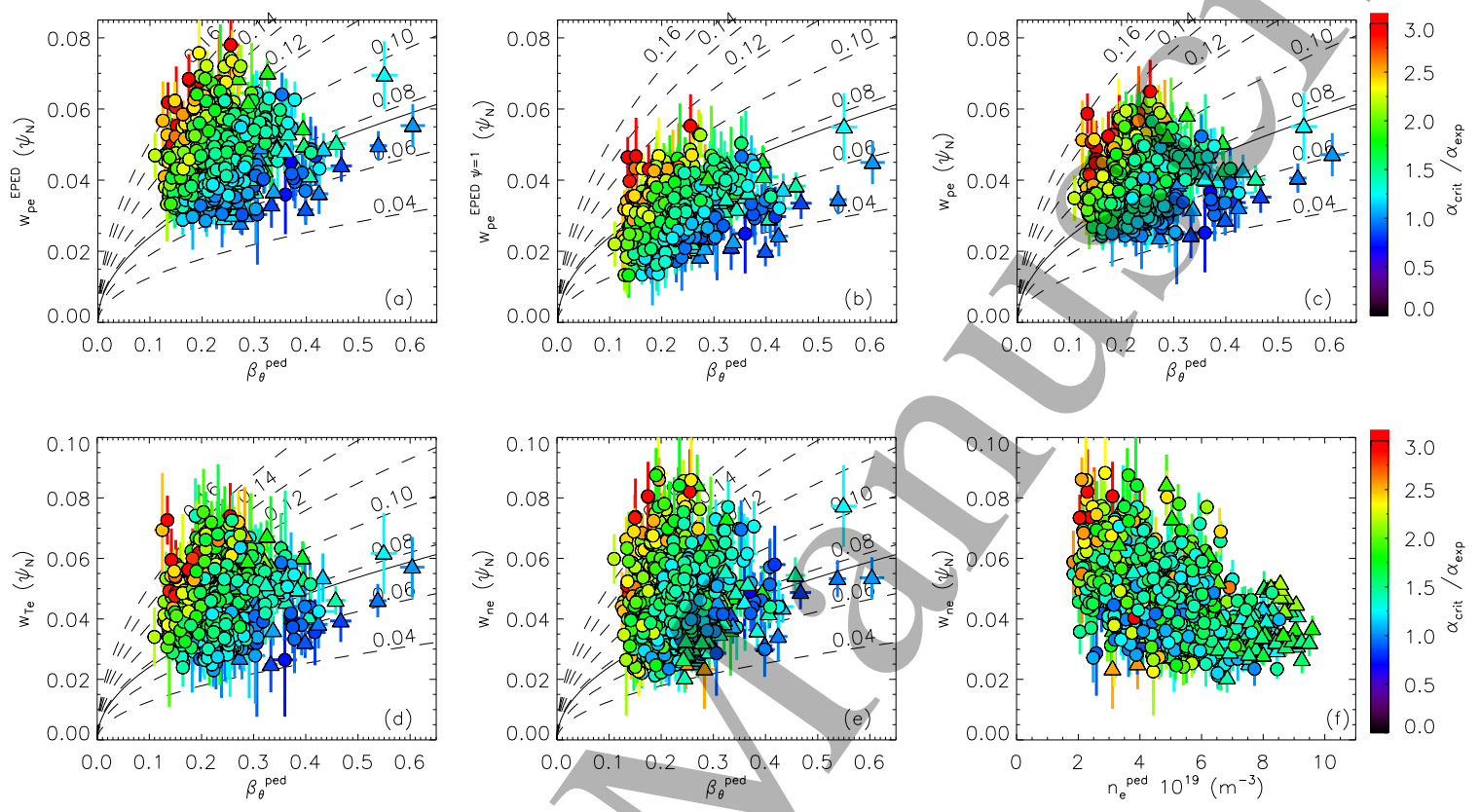

Figure 13. Correlation between the pressure pedestal width and the poloidal beta at the pedestal for the entire database. The continuous line shows the EPEDI assumption with $D=0.076$. The dashed lines highlight the trends $D\left(\beta \theta^{\text {ped }}\right)^{1 / 2}$, with the corresponding $D$ specified near the end of each line. The colors highlight the ratio $\alpha_{c r i t} / \alpha_{\text {exp. }}$. The three frames shows the three different definitions of pressure pedestal width discussed in section 3.1: the standard EPED definition (a), considering the pedestal region only till $\psi_{N}=1$ (b) and the fit to the product of the deconvolved fits (c). Frames (d) and (e) show the correlation between the width of the pedestal temperature and density with $\beta_{\theta}{ }^{\text {ped }}$. Frame (f) shows the correlation between the width of the pedestal density and $n_{e}{ }^{\text {ped }}$.

To verify how much the definition of the width of the pedestal pressure can affect the coefficient $D$, figure 13 shows the pedestal width versus the total beta poloidal at the pedestal. Each frame shows one of the three definitions discussed in section 3.1. The continuous line shows the EPED1 assumption $w_{p e}{ }^{E P E D}=0.076\left(\beta \theta^{\text {ped }}\right)^{1 / 2}$. It is clear that the JET-ILW pedestal width does not follow the $0.076 \times\left(\beta_{\theta}{ }^{\text {ped }}\right)^{1 / 2}$ dependence assumed in EPED1, no matter which definition is used. Moreover, the $D$ value is not constant and varies in the range 0.04-0.16. Therefore, we can conclude that, in general, the EPED1 assumption on the pedestal width is not 
satisfied in JET-ILW. It is still possible that such a large variation in $D$ is consistent with EPED1.6 model. However, the systematic test of the EPED1.6 model in JET-ILW is beyond the scope of this work.

Two further important results can be extracted from figure 13, both related to the distance of the pre-ELM pedestal from the PB boundary. As discussed in section 2.3, this has been quantified as the ratio $\alpha_{c r i t} / \alpha_{\text {exp }}$. When the ratio is close to 1, the ELM is triggered when the pedestal reaches the PB boundary. With $\alpha_{c r i t} / \alpha_{\text {exp }}>>1$, the ELM is triggered when the pedestal is still PB stable. The colors in figure 13 highlight the ratio $\alpha_{c r i t} / \alpha_{\text {exp }}$, with a light blue color showing the PB limited pedestal $\left(\alpha_{\text {crit }} / \alpha_{\text {exp }} \approx 1\right)$ and green/yellow/red colors a PB stable pedestal $\left(\alpha_{\text {crit }} / \alpha_{\exp }>1.5\right)$. Figure 13 shows a clear systematic pattern, in which, at constant $\beta_{\theta^{p e d}}$, the distance from the PB boundary increases with increasing pedestal width. Basically, a wide pedestal tends to be far from the PB boundary. The second interesting result is that PB limited pedestals tend to be consistent with the EPED1 assumption. As shown in figure 13(a), the data with $\alpha_{\text {crit }} / \alpha_{\text {exp }} \approx 1$ (light blue data) have a pedestal width consistent with the expression $w_{p e}{ }^{E P E D}=0.076\left(\beta_{\theta}{ }^{\text {ped }}\right)^{1 / 2}$ (the continuous black line). This is an important result because it shows that the JET-ILW pedestals that are PB limited can be correctly predicted by EPED1, both in terms of pedestal height and pedestal width.

An exhaustive discussion on $\alpha_{c r i t} / \alpha_{\text {exp }}$ is possible only after the correlation of the pedestal width (and, later, of the pedestal position) with the engineering parameters is presented. Further discussions on $\alpha_{c r i t} / \alpha_{\text {exp }}$ are presented in section 6.4 .

It is not the scope of this work to discuss the behavior of the pedestal with of temperature and density. Figures 13(d) and 13(e) show $w_{T e}$ and $w_{n e}$ respectively, mainly to highlight their range of variation. Both $w_{T e}$ and $w_{n e}$ have a qualitative behavior similar to the pressure width. At constant $\beta_{\theta^{p e d}}$, the increase of $w_{T e}$ and $w_{n e}$ is correlated to the increase of $\alpha_{c r i t} / \alpha_{\text {exp }}$. Moreover, since the gas dosing rate affects the density position (see for example references $[28,39,72]$ or later in section 5.4), it is possible that the gas dosing affects also density width. This is expected, for example, within in the neutral penetration model [67] which predicts a negative correlation between density width and height. Figure 13(f) shows the correlation of $w_{n e}$ with $n_{e}{ }^{\text {ped }}$. Perhaps, a weak negative correlation is present but the scatter of data is very. Moreover, most of the data with low $w_{n e}$ and high $n_{e}^{\text {ped }}$ are at high triangularity. So no statements can be derived from figure 13(f). A test of the neutral penetration model is outside the scope of this work.

Figure 14(a) shows the correlation of the pressure width with the absorbed power. No clear trend is observed when looking at the entire database, however clear trends emerge when the 
power scan subsets are highlighted. An example is shown in figure 14(b). The full symbols show the power scans at $1.4 \mathrm{MA}$ discussed in figure $8(\mathrm{~d})$. The pressure pedestal width $w_{p e}$ increases with increasing power. For simplicity, temperature and density widths are not shown. The $w_{p e}$ increase with increasing power seems driven mainly by the increase $w_{T e}$, rather than $w_{n e}$, but no conclusive claims are possible ( $w_{n e}$ tends to slightly increase with power as well). The open symbols in figure $14(\mathrm{~b})$ show the power scans at $2.5 \mathrm{MA}$ discussed in figure $8(\mathrm{~g})$. Albeit much weaker, also these subsets show a pedestal widening with increasing power. The weaker trend might be due to the narrower power range compared to the $1,4 \mathrm{MA}$ subsets but also to the lower q95 that might reduce the effect of the Shafranov shift. Other subsets show a similar correlation, so the increase of the pedestal width with increasing power is rather robust. The correlation between width and power has an important implication for the position of the pedestal, as discussed in section 5.4.

The correlation of the pedestal width with gas fueling rate is shown in figure 14(c). Correlations emerge only when pure gas scans are highlighted. Figure 14(d) shows the 2MA gas scans discussed in figures 10-12. A new subset, a gas scan at 1.4MA (pink data) has been added. The results suggest a widening of $w_{p e}$ with increasing fueling rate. This is consistent with the results discussed in other machines, including JET-C [25].
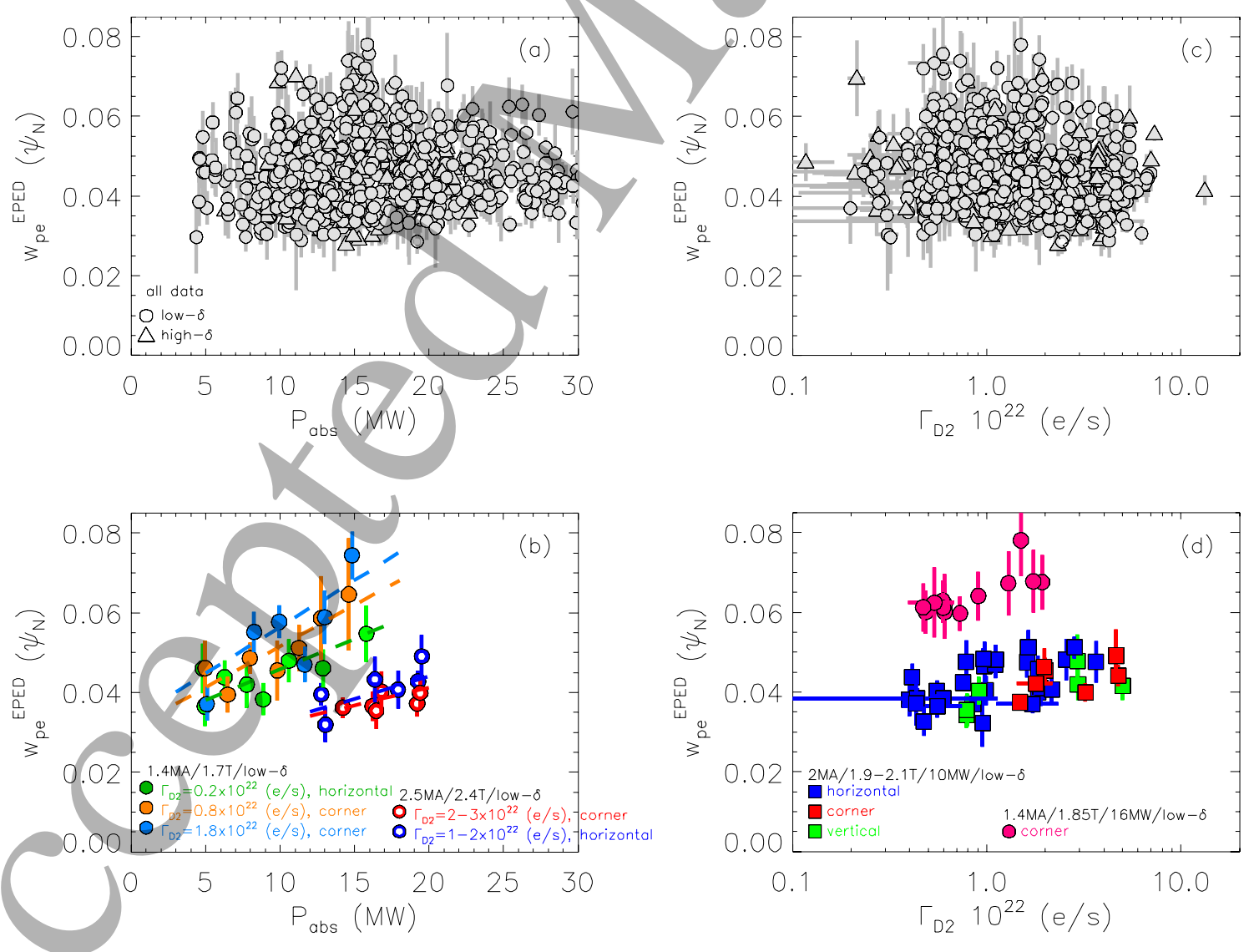
Figure 14. Correlation between the pressure pedestal width and absorbed power for (a) the entire database and for (b) power scans at 1.4MA and 2.5MA. The power scans are the same scans discussed in figures 8 and 9. The correlation between the pressure pedestal width and gas fueling rate is shown in frame (c) the entire database and in frame (d) for gas scans at 1.4MA and 2MA. The 2MA gas scans are the same as discussed in figure 10,11 and 12. The width definition is the same as in figure 13(a) and it is consistent with the JET results discussed in earlier literature.

Figure 14(b) also suggests a dependence of the pedestal width on the plasma current. For example, at $P_{a b s}=15 \mathrm{MW}$, the $2.5 \mathrm{MA}$ pedestals have $w_{p e} \approx 0.04 \psi_{N}$ while the $1.4 \mathrm{MA}$ pedestals have $w_{p e} \approx 0.06 \psi_{N}$. Unfortunately, due to lack of "clean" $I_{p}$ scans (i.e. with all other engineering parameters fixed) this remains a weak observation and no conclusive claims are possible.

As a final remark, it is important to mention that the three definitions of the pedestal width lead to similar qualitative conclusions. For a more direct comparison with the earlier JET results, the definition of figure 13(a) is used in the rest of the work. It is also important to mention that no obvious trends between the pedestal width and other dimensionless parameters, such as ( $v^{* p e d}$ and $\rho^{* \text { ped }}$ ) have been observed (even when considering specific subsets).

This subsection can be summarized with the following key points:

- In general, the pedestal pressure width of JET-ILW is not consistent with the EPED1 assumption and the coefficient $D$ varies between $0.04-0.16$.

- The JET-ILW pedestal width is consistent with EPED1 when the pedestal is PB limited.

- At constant $\beta_{\theta}{ }^{\text {ped }}$, the pedestal width is positively correlated with $\alpha_{c r i t} / \alpha_{\text {exp }}$.

- No obvious correlation between pedestal width and dimensionless parameters has been observed.

- The pedestal width increases with increasing power and increasing gas fuelling rate. The width behavior with power will have important implications for the pedestal position (see section 5.4). 


\subsection{Pedestal positions and its correlation with pedestal width and $\mathbf{n}_{e}{ }^{\text {sep }}$.}

Variations in the pedestal positions of the electron density were noticed for the first time in 1996 during a gas scan in JET [22]. Then, differences in $n_{e}^{p o s}$ and $T_{e}{ }^{p o s}$ were observed in 20092011 in DIII-D and JET-C [102, 103]. The importance of the pedestal position in the pedestal performance was highlighted in NSTX and DIII-D [124, 125, 126] and finally systematically investigated in AUG [28] and JET-ILW [39, 72].

Given the role of the pedestal position in the pedestal performance, it is important to identify the parameters that lead to the variation of $n_{e}^{p o s}$ and $T_{e}^{p o s}$. So far, the main message presented in the literature is that the key parameter is the gas fuelling rate [28, 39, 72]. This section shows that also the power has a major impact on the pedestal position. In section 6 , it will be shown that the interplay between gas dosing and power and the corresponding effect on the pedestal position is essential to understand the behavior of the pedestal height.

\subsubsection{Dependence on absorbed power.}

Figure 15 shows the correlation of (i) the pedestal position of temperature, (ii) density and (iii) the relative shift with the absorbed power. A correlation between $T_{e}^{p o s}$ and $P_{a b s}$ can be observed even looking at the entire database, see figure 15(a). At low power, $T_{e}^{\text {pos }}$ is located more outward than at high power. This behavior is extremely clear when looking at the 1.4MA power scan, shown in figure 15(b). Interestingly, no major difference can be observed between the power scan at low gas (orange data) and the power scan at high gas (light blue data). The 2.5MA power scan shows a similar trend even if significantly weaker due to the narrower power range, see figure 15(c). An example of the $T_{e}$ profiles at low and high power is shown in figure 16. In the high power pulse, figure 16(c), the position of maximum gradient of the temperature is clearly more inward than in the low power pulse, figure 16(a). Note that this result is not affected by the uncertainty in $T_{e}^{\text {sep }}$. The shaded areas in figures 16(b) and 16(d) highlight the variation of $T_{e}^{p o s}$ due to a $10 \%$ uncertainty in $T_{e}^{\text {sep }}$. The variation of $T_{e}^{\text {pos }}$ is minimal.

The density position, instead, has no strong correlation with the power, as shown in figures 15(d), 15(e) and 15(f). However, there is a significant variation in $n_{e}{ }^{p o s}$ with the gas fueling rate. The power scan at low gas has $n_{e}^{\text {pos }} \approx 0.995 \psi_{N}$ while the power scan at high gas has the density located more outward, at $n_{e}{ }^{p o s} \approx 1.005 \psi_{N}$. This behavior will turn out to be very important in the discussion of the non-PB limited pedestal in section 6.4.

Finally, the correlation of the relative shift with the power is shown in figures $15(\mathrm{~g}), 15(\mathrm{~h})$ and 15(i). An increase of the relative shift with power can be observed. This is also clear for the profiles shown in figure 16 . The positive correlation of the relative shift with the power is 
due to the inward shift of $T_{e}^{\text {pos }}$. Moreover, it is very important to highlight that the power scan at high gas, light blue data in figure 15(h), reaches relative shifts higher than the power scan at low gas, green data in figure $15(\mathrm{~h})$. This is due to the more outward density position in the high gas case.

Actually, the behavior of the pedestal position is more complex and at least another engineering parameter, the divertor configuration, is likely to play an important role. This is shown in figure 15(f), where the correlation between $n_{e}^{p o s}$ and $P_{a b s}$ is shown for the $2.5 \mathrm{MA}$ power scans. The low gas fueling scan (blue data) has a density pedestal position more outward than the high gas fueling scan (red data). This is likely due to the different divertor configuration. The blue subset has outer strike point on the horizontal target, so, despite the lower gas dosing, the neutral pressure is likely higher than in the red subset, where the outer strike point is in the corner. This hypothesis is verified at the end of section 5.4.3 using the separatrix density as a proxy for the neutral pressure.

\subsubsection{Dependence on gas dosing rate.}

Due to the role of the divertor configuration, the results related to the gas dosing discussed in figure 15(e) are not conclusive. Therefore, figure 17 discusses more in detail the correlation of the pedestal position with $\Gamma_{D 2}$. The top frames of figure 17 show the correlation for the entire database. The density position seems to have a weak positive trend with $\Gamma_{D 2}$, figure $17(\mathrm{c})$. The bottom frames highlight four specific subsets. Each subset has been selected to have constant engineering parameters (including the divertor configuration), apart from the gas dosing rate.

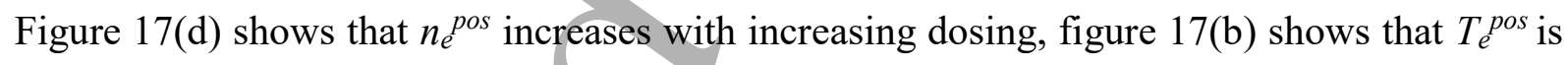
not strongly dependent on $\Gamma_{D 2}$. Consequently, the relative shift increases with the gas dosing rate, figure 17(f). As an example, figure 18 shows the density and temperature profiles for two pulses with low and high $\Gamma_{D 2}$.

\subsubsection{On the origin of the variations in $T^{\text {pos }}$ and $n e^{p o s}$.}

Figures 15 and 17 have shown that $T_{e}^{\text {pos }}$ moves inward with increasing power and $n_{e}^{\text {pos }}$ moves outwards with increasing gas fueling rate.

The change in $T_{e}^{\text {pos }}$ with power is clearly due to the widening of the pedestal. This can be seen qualitatively from the profiles of figure 16. More quantitatively, figure 19(a) shows the correlation between $T_{e}{ }^{\text {pos }}$ and the width of the pedestal temperature. A trend is present even looking at the entire database (grey data) and it is particularly clearly when specific subsets are highlighted. For simplicity, figure 19(a) shows only the 1.4MA/high-gas/corner subset. With 
$T_{e}{ }^{s e p}=100 \mathrm{eV}$, the widening of the pedestal width with increasing power (section 5.3) leads to the inward shift of the pedestal temperature. Within the EPED model, the widening of the width is due to the increase of the poloidal beta at the pedestal. This is tested in figure 19(b), where the correlation between $T_{e}^{p o s}$ and $\beta \theta^{\text {ped }}$ is shown for the low- $\delta$ pulses. The correlation is not very clear. On the other hand, the correlation is clear once subsets with similar $\alpha_{\text {crit }} / \alpha_{\text {ped }}$ are considered [data with similar colors in figure 19(b)]. In particular, the subset near the PB boundary (blue data, with $\alpha_{\text {crit }} / \alpha_{\text {ped }} \approx 1$ ) show that the increase of the poloidal beta leads to the inward shift of the temperature. This is clearly due to the pedestal widening with increasing $\beta \theta^{\text {ped }}$ [see figure 13]. We can conclude that when the pedestal is near the PB boundary, EPED can qualitative describe the behavior of the pedestal position of the temperature. However, at constant $\beta \theta^{\text {ped }}$ an inward shift of the temperature can be observed with increasing $\alpha_{\text {crit }} / \alpha_{\text {exp }}$. This suggests that further transport mechanisms (on top of the standard KBM constraint assumed in EPED) must be invoked to explain the pedestal widening and the corresponding $T_{e}{ }^{\text {pos }}$ inward shift. This point is further discussed in section 8.3.

Instead, the variation in $n_{e}^{\text {pos }}$ cannot be ascribed to the same mechanism. $n_{e}^{p o s}$ and width of the pedestal density are not correlated, as shown in figure 19(c). Indeed, AUG results [28, 127] have shown that the outward shift of $n_{e}$ pos is due to a significant increase of the density at the separatrix. The idea that the density position is correlated to the SOL and/or separatrix density is very appealing because it might provide a link between fueling, recycling, divertor configuration and neutral pressure with density position and pedestal performance. To investigate this hypothesis, the separatrix density has been used as simple proxy for the neutral pressure (see section 5.2). Figure 20(a) shows the correlation between $n_{e}{ }^{\text {sep }}$ and $n_{e}{ }^{\text {pos }}$ for the entire database and for the 2MA subset (i.e. same subset of figure 10-12 and 17). A clear correlation between $n_{e}^{\text {sep }}$ and $n_{e} e^{\text {os }}$ is present and all the three JET-ILW subsets are well aligned, despite the different divertor configuration. Note that also the 2MA JET-C subset (black squares) is well correlated with the rest of the data. The JET-C subset has no gas injection, so low neutral pressure and hence very low $n_{e}{ }^{\text {sep }}$. As a consequence, the density pedestal is located more inward than in JET-ILW, with $n_{e}{ }^{p o s}<1.00 \psi_{N}$. The results of figure 20 are therefore consistent with the hypothesis that the density shift is due to a change in $n_{e}{ }^{\text {sep }}$ driven by a change in fueling and recycling. It is therefore not likely to find a simple scaling of $n_{e}{ }^{\text {pos }}$, as many factors like fueling location, divertor configuration, $I_{p}$, power density in the SOL can play a role.

Note that, the data from the entire database in figure 20(a) still show a significant scatter. This might be due to at least two reasons. First of all, $n_{e}{ }^{\text {sep }}$ might not be dependent only on the neutral 
pressure, but perhaps also on plasma current and power [112]. Indeed, figure 20(b) suggests that the plasma current might be important. The scatter is reduced once $n_{e}{ }^{\text {sep }}$ is normalized by $I_{p}$. A further investigation on the parameters that affects $n_{e}{ }^{\text {sep }}$ is presented in section 7. Second, we cannot either exclude that $n_{e}{ }^{\text {sep }}$ is a too crude approximation for the neutral pressure.

As a final comment, it is useful to investigate if any systematic difference in $n_{e}^{\text {sep }}$ and $n_{e}^{p o s}$ is present between divertor configurations. Figure 20(c) shows the distribution of $n_{e}^{\text {sep }}$ for the entire database, separated by the three divertor configurations. The overlap between the three histograms is significant, however the $n_{e}{ }^{\text {sep }}$ distribution for the horizontal configuration is peaked at higher $n_{e}^{\text {sep }}$ than for the corner configuration. Given the empirical role of $n_{e}{ }^{\text {sep }}$ in the pedestal performance (figure 12), this result is likely correlated with the different pedestal pressure height observed in different divertor configurations, as discussed in $[30,31]$. Due to the limited amount of pulses, no statements are possible for the vertical configuration. Finally, figure 20(e) shows the distributions of $n_{e}^{p o s}$. In this case, the difference is minimal, with the peak of the distribution located at $\psi_{\mathrm{N}}=1.0$ for the corner configuration (red line) and only slightly more outward for the horizontal configuration (blue data).

This subsection can be summarized with the following main results:

- $T_{e}{ }^{p o s}$ moves inwards with increasing power.

- This is due to a widening of the pedestal with power.

- When the pedestal is near the PB boundary, this behavior is well explained within the EPED model. When $\alpha_{\text {crit }} / \alpha_{\text {exp }}$, further transport mechanism must be invoked.

- $n_{e}^{p o s}$ moves outwards with increasing fuelling. The divertor configuration also seems to play a role.

- $n_{e}^{p o s}$ is strongly correlated with $n_{e}^{\text {sep }}$. The correlation is very clear with both gas fuelling scans and divertor configuration scans and holds for both JET-ILW and JETC subsets.

- This is consistent with the hypothesis that the change in $n_{e}{ }^{p o s}$ is driven by a change in the neutral pressure due to a variation in fuelling and recycling. 

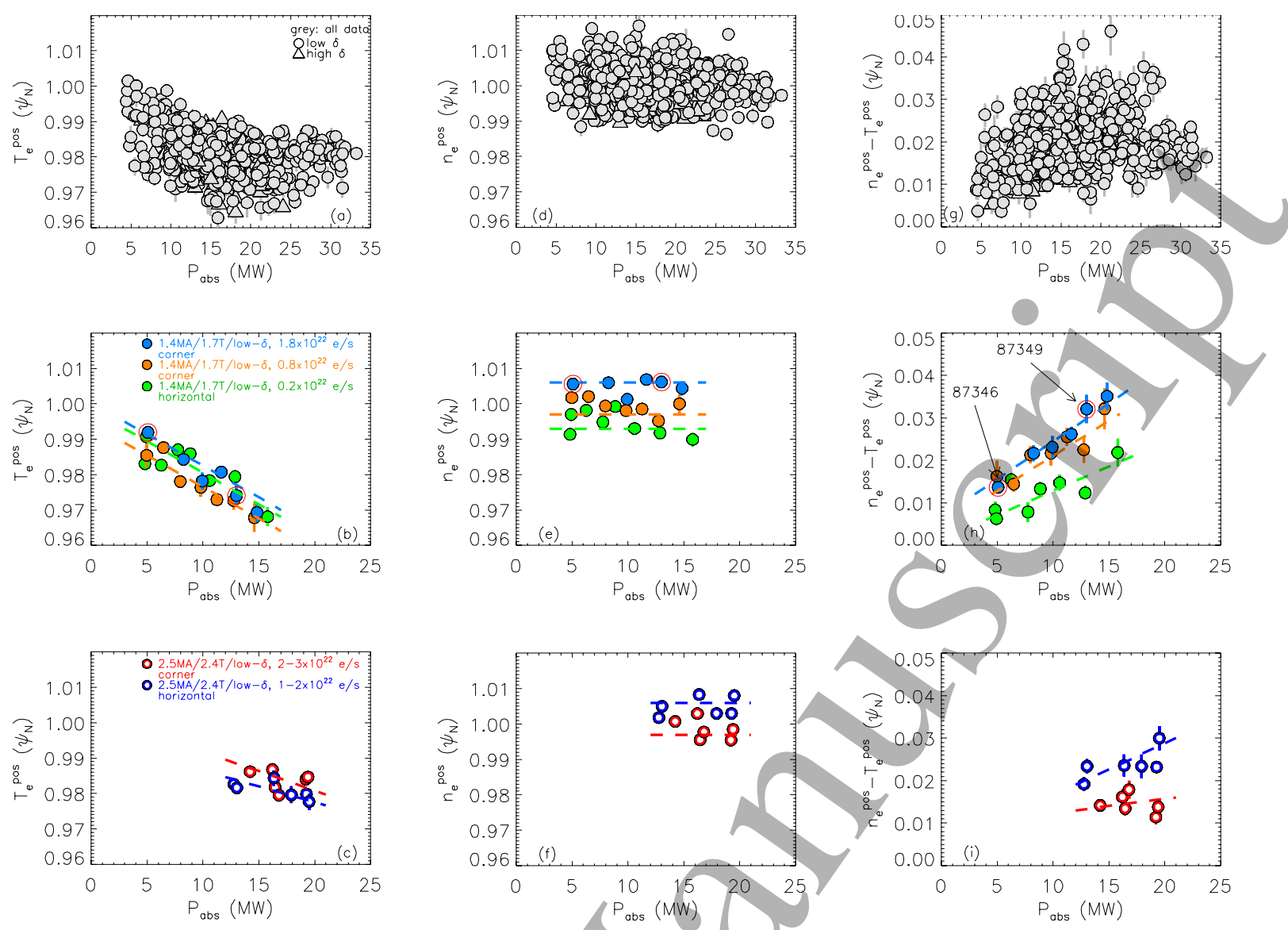

Figure 15. Correlation between the pedestal position of electron temperature (a), density (d) and relative shift (g), with the absorbed power for the entire database. Frames (b), (e) and (h) show the same correlation for the 1.4MA power scans discussed in figure $8(d)$. Frames (c), (f) and (i) show the correlation for the 2.5MA power scans discussed in figure $8(\mathrm{~g})$. The red circles in frames (b), (e) and (h) highlight the two pulses (at low power and at high power) used in figure 16 to show an example of the temperature and density profiles. 

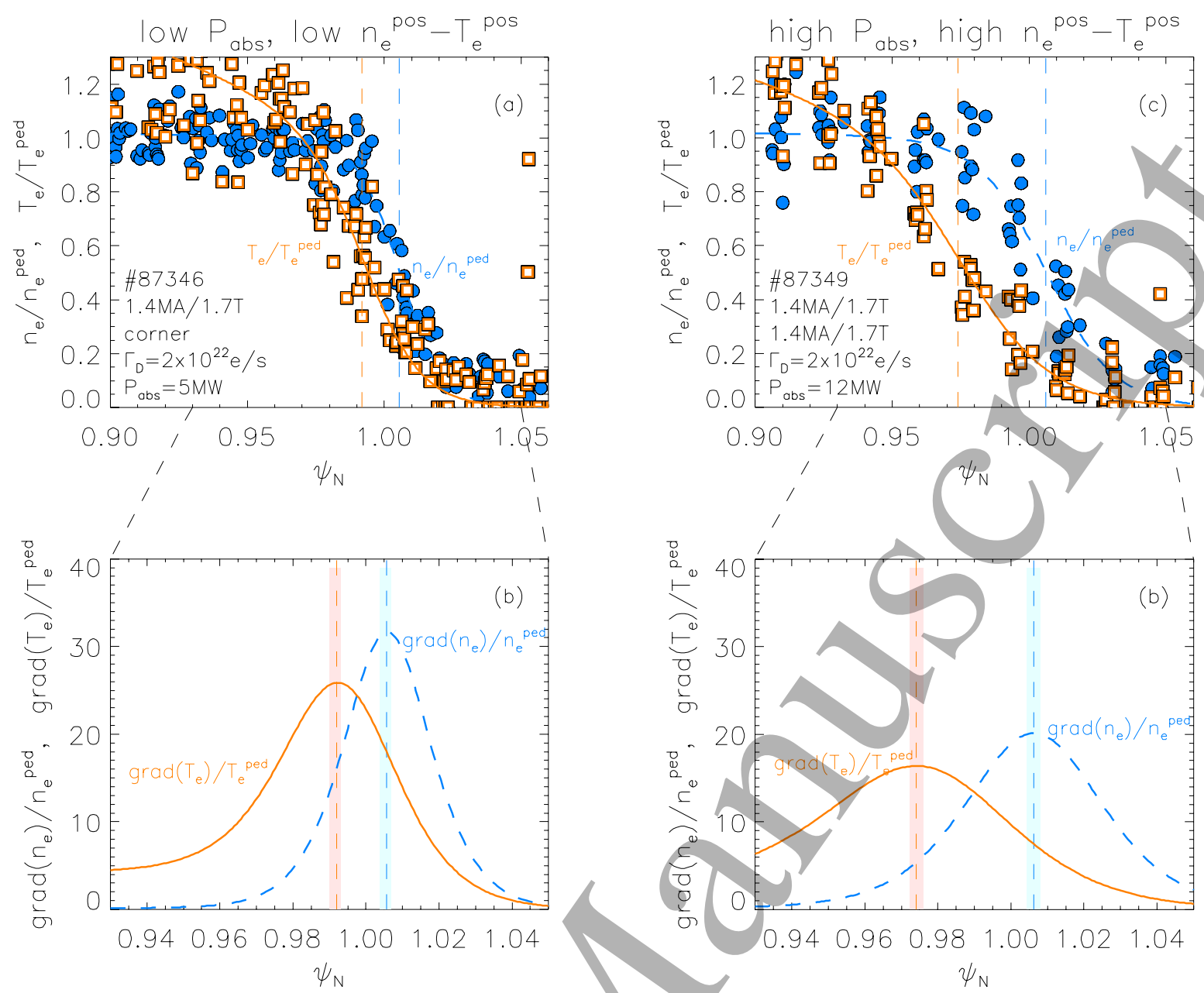

Figure 16. (a) Temperature and density profile for the low power pulse highlighted by a red circle in figure 15. The profiles are normalized to the pedestal top value to show more clearly the difference between temperature and density position. (b) Corresponding density and temperature gradients. (c) Temperature and density profile for the high power pulse highlighted by a red circle in figure 15 and (d) corresponding density and temperature gradients. The vertical dashed lines in frames (b) and (d) highlight the position of the maximum gradient. The shaded areas highlight the variation in $T_{e}^{\text {pos }}$ and $n_{e}{ }^{\text {pos }}$ due to a $10 \%$ uncertainty in $T_{e}^{\text {sep }}$. 

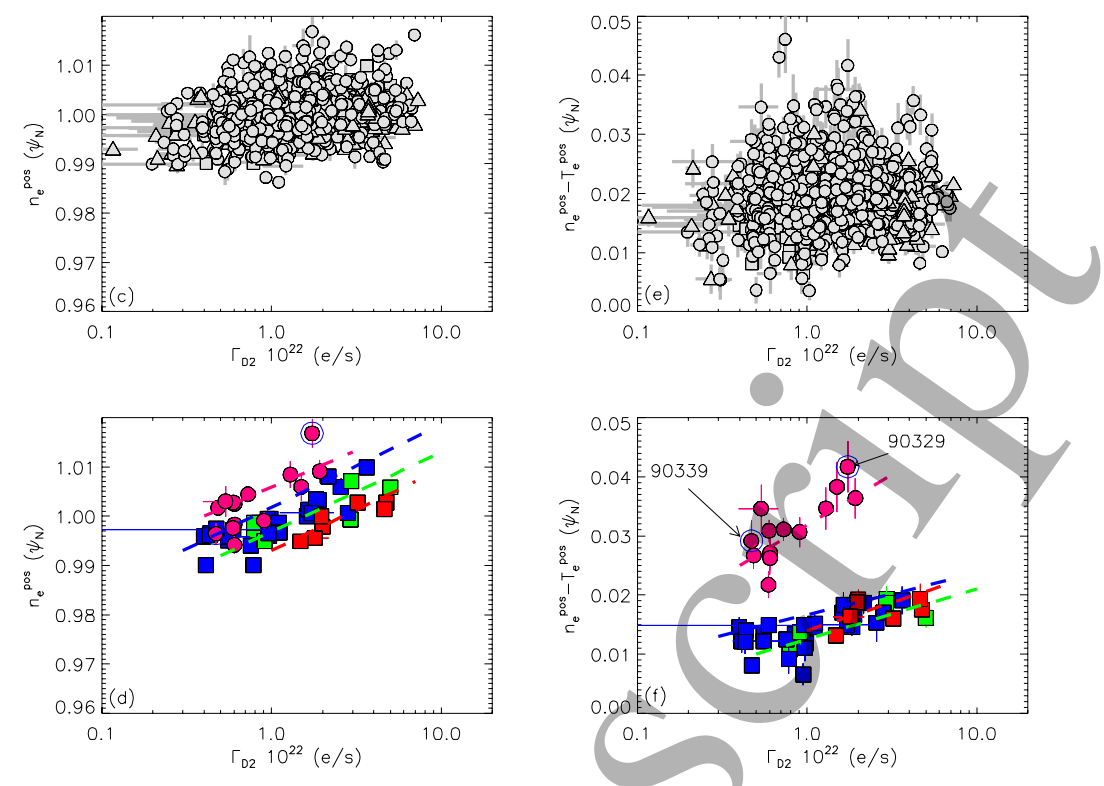

Figure 17. Correlation between $T_{e}^{\text {pos }}(a), n_{e}^{\text {pos }}(c)$ and relative shift (e), with the gas fueling rate for the entire database. Frames (b), (d) and (f) show the same correlation for the $2 \mathrm{MA}$ subsets discussed in figures 10-12 (red, blue and green squares) plus a new subset at 1.4MA (selected with constant engineering parameters apart from the gas fueling rate). The red circles in frames (b), (d) and (f) highlight the two pulses (at low fueling and at high fueling) used in figure 18 to show an example of the temperature and density profiles. 

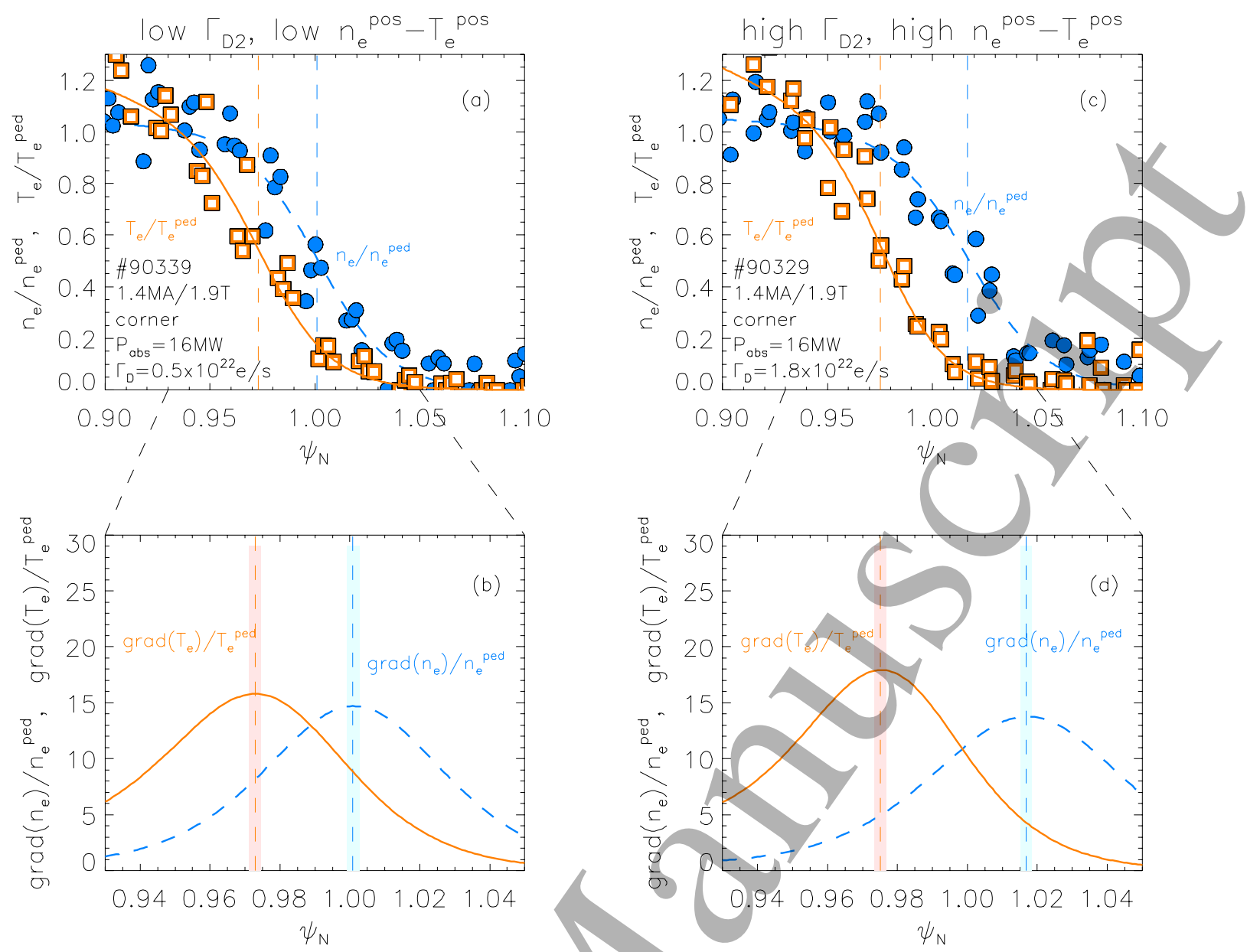

Figure 18. (a) Temperature and density profile for the low gas fueling pulse highlighted by a red circle in figure 17. The profiles are normalized to the pedestal top value to show more clearly the difference between temperature and density position. (b) Corresponding density and temperature gradients. (c) Temperature and density profile for the high gas fueling pulse highlighted by a red circle in figure 17 and (d) corresponding density and temperature gradients. The vertical dashed lines in frames (b) and (d) highlight the position of the maximum gradient. The shaded areas highlight the variation in $T_{e}^{\text {pos }}$ and $n_{e}^{\text {pos }}$ due to a $10 \%$ uncertainty in $T_{e}^{\text {sep }}$. 

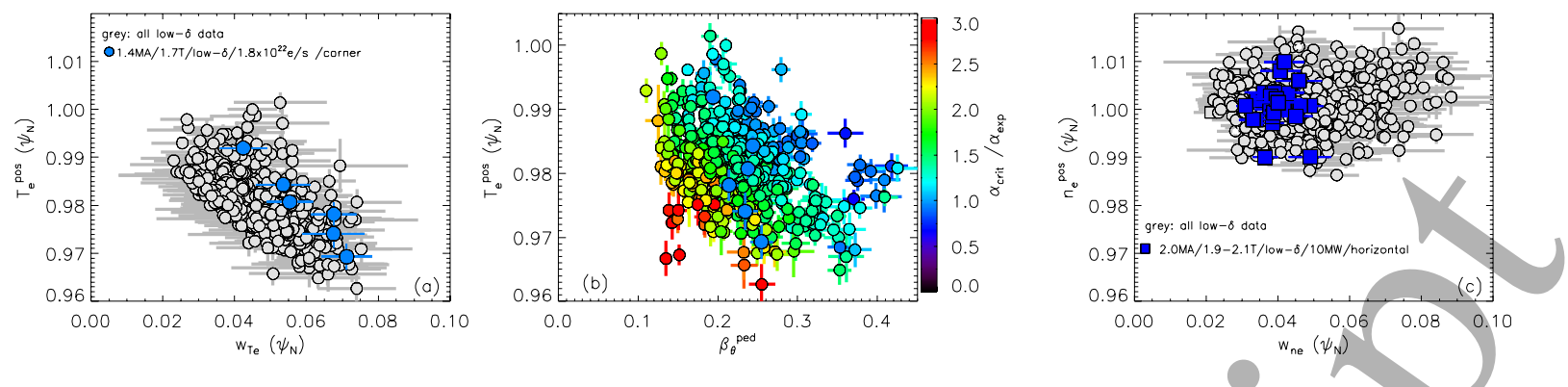

Figure 19. (a) Correlation between position of the pedestal temperature and the corresponding pedestal width for the entire database (grey data, only low- $\delta$ pulses for simplicity) and for the 1.4MA power scan at high gas fueling rate discussed in figures 8, 9, 14, 15. (b) Correlation between $T_{e}^{\text {pos }}$ and the pedestal poloidal beta. Colors highlight the ratio $\alpha_{c r i t} / \alpha_{\text {exp. }}$ (c) Correlation between position of the pedestal density and the corresponding pedestal width for the entire database (grey data) and for the 2MA gas scan with OSP on the horizontal target discussed in figures 10-12.

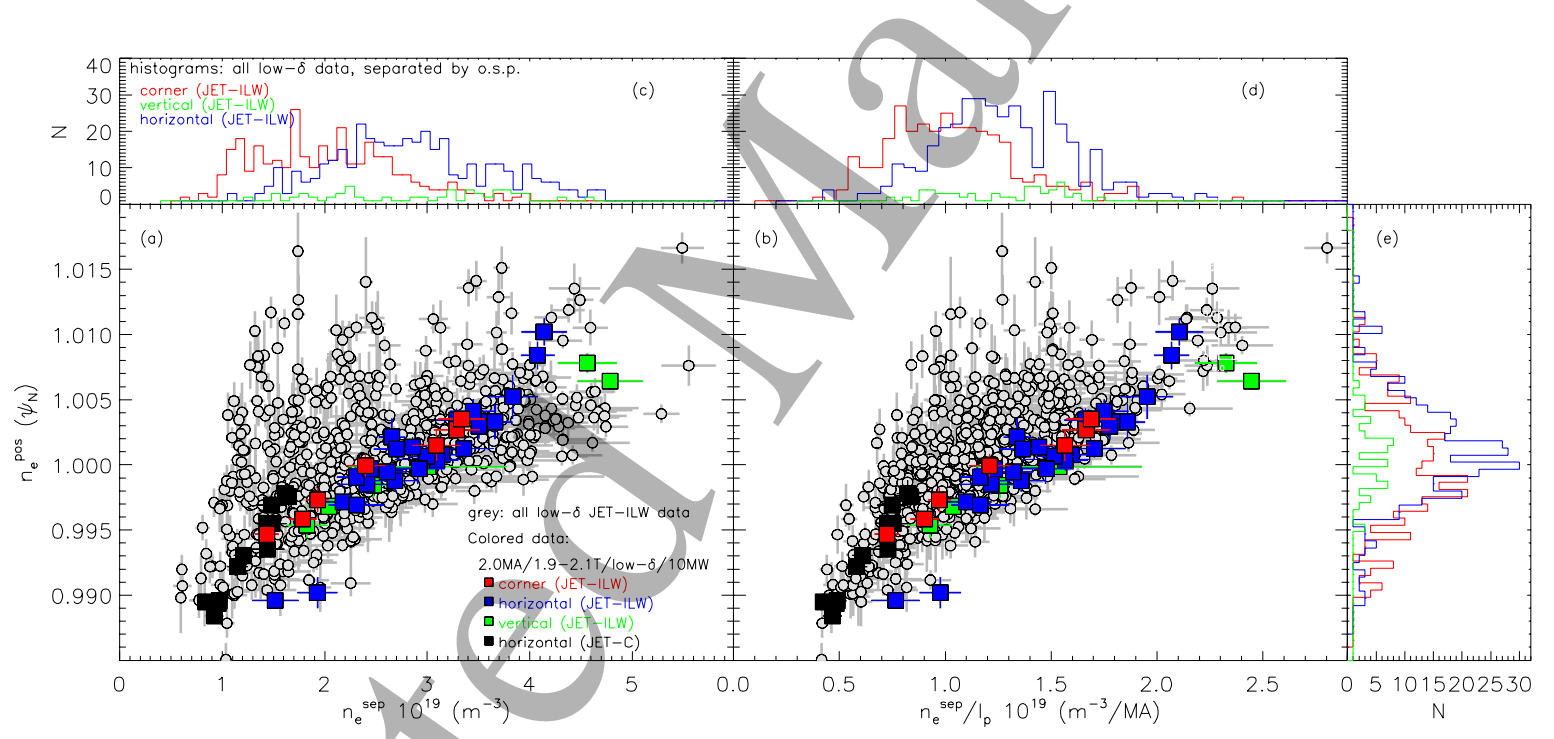

Figure 20. (a) Correlation of the separatrix density and of (b) the separatrix density normalized to the plasma current with the position of the pedestal density. Grey data highlight the entire database (only low- $\delta$ pulses for simplicity). The squares highlight the 2MA subsets discussed in figures 10-12. Frames (c), (d), (e) show respectively the distributions of separatrix density, separatrix density normalized to $I_{p}$ and density position. 


\section{NORMALIZED PRESSURE GRADIENT AND COMPARISON WITH THE PEELING-BALLOONING STABILITY.}

This section discusses some key characteristics of the experimental normalized pressure, $\hat{\alpha}_{\text {exp }}$, and compares it with the normalized pressure gradient predicted by the peeling ballooning theory, $\alpha_{\text {crit. }}$ In the literature, this type of analysis can be found only for very limited subsets of data due to the highly time consuming processes of the pre-ELM profile analysis and PB stability analysis. Therefore, the first goal of the section is to verify the reliability of $\alpha_{\text {exp }}$ and $\alpha_{\text {crit }}$ contained in the database. This is done in section 6.1 and section 6.2 by testing some earlier results that were obtained using few pulses. Note that this exercise does not lead only to a test of the reliability of the database, but it strengthens also the earlier results by extending them to a much wider set of data. The second goal of the section is to discuss the difference in the pressure gradient between high and low triangularity plasmas. This is done in section 6.3. The last goal of the section is to investigate the discrepancy between $\alpha_{\text {exp }}$, and $\alpha_{c r i t}$. This is done in section 6.4, where the experimental conditions for which the JET-ILW pedestal is not PB limited are identified.

For simplicity, $\alpha_{c r i t}$ has been determined using $j_{b s}$ from the Sauter formula. However, as shown in figure 4, the use of the Hagar formula in determining jbs leads to a minimal change in $\alpha_{c r i t}$, with $0.9<\alpha_{\text {crit }}^{\text {hager }} / \alpha_{\text {crit }}^{\text {sauter }}<1.2$. Such a small variation does not affect the conclusions presented in this section.

\subsection{Correlation between $\alpha_{\exp }$ and dimensionless parameters.}

Dimensionless parameters such as the normalized total pressure and the pedestal collisionality can have a significant impact on the stability of the PB modes [47]. Assuming that the pedestal can be described by the PB model, $\alpha_{\exp }$ is then supposed to depend on both $\beta_{\mathrm{N}}$ and $v^{*}$. Within the PB framework, no dependence on $\rho^{*}$ is expected.

Figure 21(a) shows the correlation between $\alpha_{\exp }$ and $\beta_{N}$. For simplicity, only low- $\delta$ plasmas are shown. According to the theory, the increasing $\beta_{\mathrm{N}}$ stabilizes the ballooning modes, shifting the ballooning boundary to higher $\alpha$ and hence increasing the pressure gradient [47]. Therefore, a positive correlation between $\alpha_{\exp }$ and $\beta_{\mathrm{N}}$ is expected. Indeed, figure 21(a) shows the increase of $\alpha_{\exp }$ with increasing $\beta_{\mathrm{N}}$, even looking at the entire database (grey data). The trend is more clear once subsets at constant collisionality are highlighted. The positive trend is strong at low collisionality (see the black diamonds) but it weakens considerably at high collisionality (see the light blue diamonds). This is consistent with the observations in two specific dimensionless 
$\beta_{\mathrm{N}}$ scan in JET-ILW [54]. The weak increase of $\alpha_{\exp }$ with increasing $\beta_{\mathrm{N}}$ at high collisionality might also be linked with the results from dimensionless $\beta$ scans obtained JT-60U and AUG $[128,129]$, where a decrease of the normalized energy confinement with increasing $\beta$ was observed. In reference [129], this was ascribed to a lack of increase of the pedestal with increasing $\beta$.

The different behavior between the low and high collisionality in figure 21(a) is linked to the behavior of the pressure in figure 8(i), where no strong correlation between $p_{e}^{\text {ped }}$ and power was observed on the subset in horizontal configuration (high collisionality), while a clear positive correlation was observed for the subset in the corner (low collisionality).
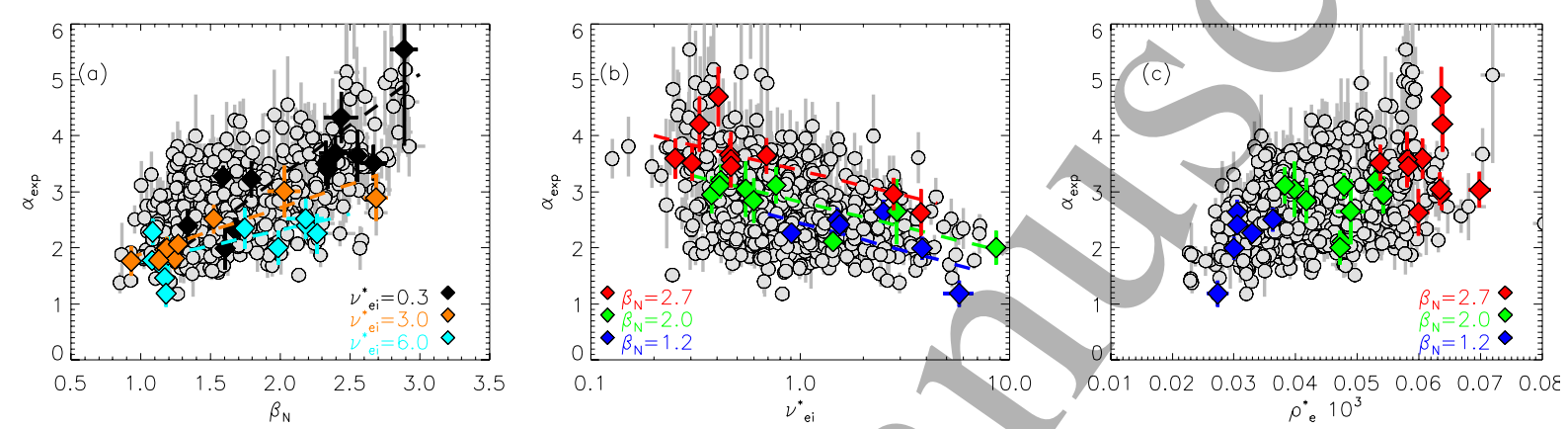

Figure 21. Correlation between the experimental normalized pressure gradient and (a) normalized total pressure, (b) normalized electron-ion collisionality at the pedestal and (c) normalized electron gyro-radius at the pedestal. Only low triangularity pulses are shown. The non-grey subsets highlight data with constant collisionality (a) or constant $\beta_{N}(b)$ and (c).

The correlation between $\alpha_{\exp }$ and pedestal collisionality is shown in figure 21(b) for low- $\delta$ plasmas. A negative trend is present. This can be qualitatively explained within the PB framework if the ballooning boundary is not steep. In this case, the reduced $v^{*}$ leads to an increase in $j_{b s}$ which in turn lead to an increase in $\alpha_{c r i t}$. Note that the negative trend is not very strong. This could be because the PB boundary is not strongly shaped in low- $\delta$ plasmas, so an increase in $j_{b s}$ can lead only to small variations in $\alpha_{c r i t}$. An important result of figure 21(b) is that in JET-ILW low- $\delta$ plasmas only a significant reduction in collisionality leads to a significant increase in the normalized pressure gradient. A factor 2 reduction in $v^{*}$ produces only a minimal increase in $\alpha_{\text {exp }}$.

The stability of the PB modes is supposed not to depend on the normalized Larmor gyroradius, as theoretically predicted and also as experimentally observed in JET-C, DIII-D and JT$60 \mathrm{U}[63,102,103,130]$. Instead, the JET-ILW data show in figure 21(c) seem to suggest a positive correlation between $\alpha_{\exp }$ and $\rho_{e}^{*}$. However, this correlations is misleading and it is due to the fact that $\beta_{\mathrm{N}}$ and $\rho^{*}$ are correlated in the database. The lowest $\rho_{e}^{*}$ plasmas are typically 
achieved at high plasma current. Most of the high $I_{p}$ pulses are not fully optimized, so, in general, they tend to have low $\beta_{\mathrm{N}}$. As an example, three subsets with constant $\beta_{\mathrm{N}}$ have been highlighted in figure $21(\mathrm{c})$. The highest $\beta_{\mathrm{N}}$ subsets also has high $\rho_{e}^{*}$. So, despite being misleading, figure 21(c) is actually consistent with the results from other machines and no obvious correlation between $\alpha_{\exp }$ and $\rho_{e}^{*}$ is present.

This subsection can be summarized with the following main results:

- $\alpha_{\text {exp }}$ increases with increasing $\beta_{\mathrm{N}}$. The trend is strong in low collisionality plasmas and very weak in high collisionality plasmas.

- $\alpha_{\text {exp }}$ increases with decreasing collisionality.

- No dependence between $\alpha_{\exp }$ and $\rho^{*}$ has been observed at constant $\beta_{\mathrm{N}}$.

\subsection{Correlation between $\alpha_{\exp }$ and pedestal position.}

In the literature, two key results are related to pedestal position. Specifically, one is related the pressure position [28] and one to the relative shift [72].

Concerning the pressure position, from a theoretical point of/view the outward shift of the pedestal pressure is supposed to lead to an outward shift of the $j_{b s}$ peak which, in turn, should have a destabilizing effect on the ballooning modes [104]. It should be pointed out that this phenomenon can be experimentally observed only if the ELM is triggered when the pedestal reaches the $\mathrm{PB}$, i.e. when the pedestal is $\mathrm{PB}$ limited. Indeed, this behavior has been experimentally observed in AUG [28] and more recently in TCV [29] and JET-ILW [39]. However, the JET-ILW results have been obtained for a very limited dataset composed of only three pulses.

To strengthen the JET-ILW result, the database has been used to verify the dependence of $\alpha_{\exp }$ on the pressure position. Figure 22(a) shows the correlation between $\alpha_{\exp }$ and $p_{e}^{\text {pos }}$ for all the low triangularity pulses of the database. When considering the entire database, no correlation between $\alpha_{\text {exp }}$ and $p_{e}^{\text {pos }}$ is observed. This is actually expected because (i) the range in $\beta_{\mathrm{N}}$ is very wide and (ii) a large part of the JET-ILW pulses are not PB limited [27, 39, 52]. Therefore, figure 22(b) highlights a subset with $\alpha_{c r i t} / \alpha_{\text {exp }} \approx 1$ and with roughly constant $\beta_{\mathrm{N}}$. The decrease of the normalized pressure gradient with the outward shift of the pressure position is clear. Note that the light blue pulses of figure 22(b) are characterized by a large variation in the gas fuelling rate, which is necessary to change the pedestal density position (see figure 17). To emphasize the agreement with the theoretical interpretation, figure 22(b) shows $\alpha$ predicted with Europed [123]. Initially, the code has been run using as inputs the experimental parameters 
corresponding to pulse 84792. The predicted $\alpha$ is consistent with the experimental one $\left(\alpha_{c r i t}=4.7\right.$ and $\left.\alpha_{e x p}=4.8 \pm 0.4\right)$. Next, the modelling has been repeated using a different $n_{e}{ }^{\text {pos }}$. The predicted $\alpha$ and the corresponding position of the pedestal pressure are shown in figure 22(b) with black dots. The modelled trend is consistent with the experimental trend, confirming the interpretation of the JET-ILW results discussed in reference [39].
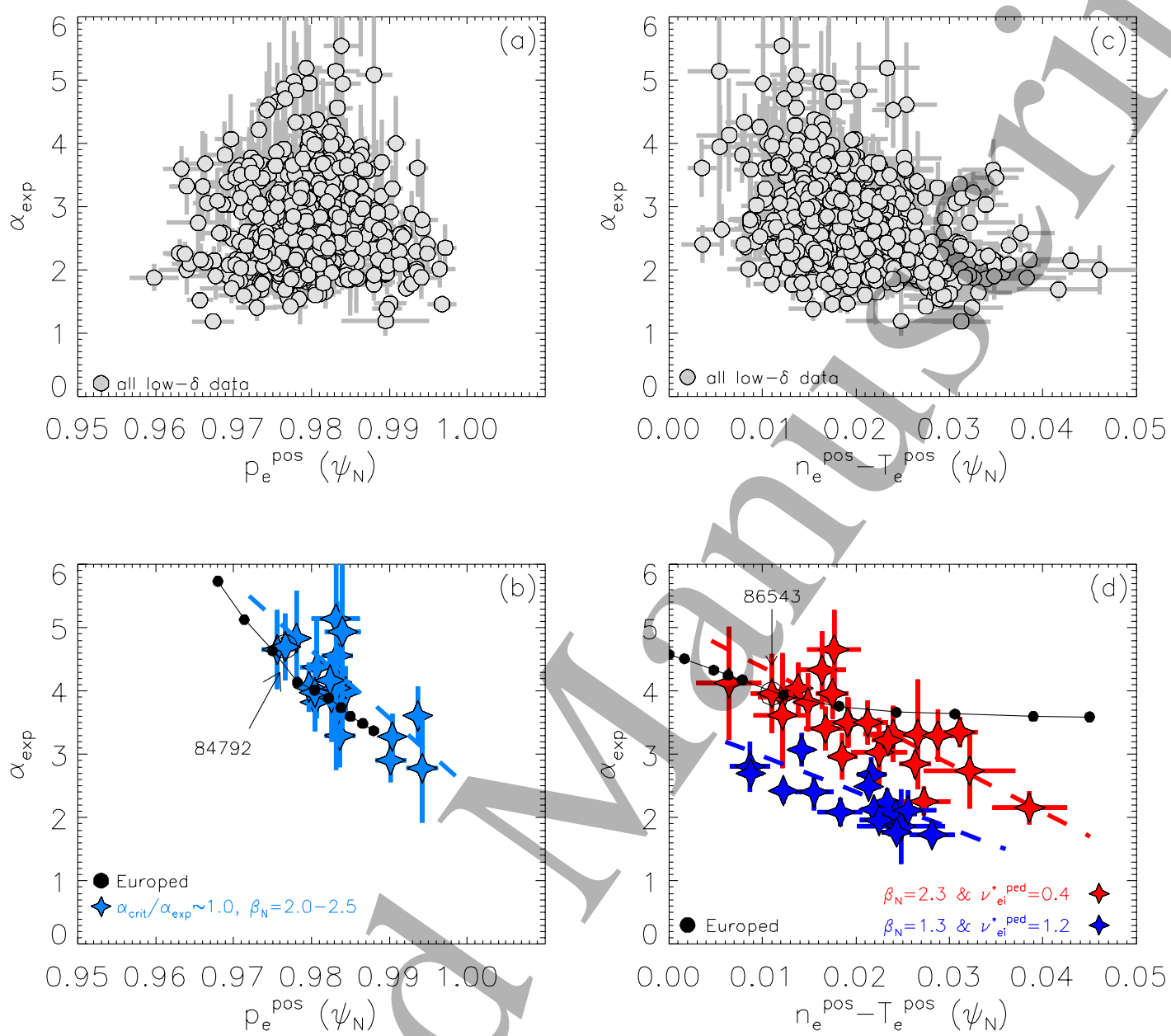

Figure 22. (a) Correlation between the experimental normalized pressure gradient $\alpha_{\text {exp }}$ and position of the pedestal pressure for the entire database and (b) for a specific PB limited subset with similar $\beta_{N}$ (blue stars). The black dots show the results modelled with Europed for pulse 84792 using different values of $n_{e}^{\text {pos }}$. (c) Correlation between $\alpha_{\text {exp }}$ and relative shift for the entire database and (b) for two subsets with similar $\beta_{N}$ and collisionality. The black dots show the results modelled with Europed for pulse 86543 using different values of $n_{e}^{\text {pos. }}$.

Concerning the relative shift, only empirical results have been obtained so far. The main message is that the increase of the relative shift is correlated with a reduction of the normalized pressure gradient [72]. At present, no theoretical explanation for this behavior is available and the PB model fails to reproduce the experimental trend [72]. This is discussed also in figure 22(d), where the correlation between $\alpha_{\exp }$ and the relative shift is shown. Two subsets are highlighted (each subset characterized by similar values of $\beta_{\mathrm{N}}$ and collisionality). 
Experimentally, the negative trend is evident in both subsets. The Europed predictions are shown in figure 22(d) by black dots. The modelling has been performed starting from the input parameters corresponding to pulse 86543 . For this pulse, the predicted $\alpha$ is consistent with the experimental one $\left(\alpha_{c r i t}=3.9\right.$ and $\left.\alpha_{e x p}=4.0 \pm 0.5\right)$. Next, the modelling has been repeated using a different pedestal density position. The model shows a negative correlation between $\alpha_{c r i t}$ and relative shift till $n_{e}{ }^{\text {pos }}-T_{e}^{p o s} \approx 0.015 \psi_{\mathrm{N}}$ and then, the trend saturates. The difference between modelled and experimental $\alpha$ is approximately a factor two at $n_{e}{ }^{p o s}-T_{e}{ }^{p o s} \approx 0.04 \psi \mathrm{N}$. As discussed in detail in section 6.4, this result is extremely robust: at high relative shift the PB model is not able to correctly describe the pedestal behavior. This point will be used in section 6.4 to understand the correlation between pedestal pressure and power discussed in figure 8 . The fact that the PB model cannot predict any change in $\alpha_{\exp }$ at $n_{e}{ }^{\text {pos }}-T_{e} e^{\text {pos }}>0.02-0.03 \psi_{\mathrm{N}}$ is because at high relative shift the density shift does not affect the pressure (see Appendix 2 and also reference [39]).

This subsection can be summarized with the following main results:

- $\quad \alpha_{\text {exp }}$ decreases with the outward shift of $n_{e}^{p o s}$ and $p_{e}^{p o s}$, as long as the pedestal is PB limited. This is consistent with AUG results and strengthens the JET-ILW results that were based on a limited dataset.

- $\quad \alpha_{\text {exp }}$ decreases with increasing relative shift, confirming earlier JET-ILW results. The PB model is not able to reproduce the experimental trend at high relative shift.

\subsection{Normalized pressure gradient in low and high triangularity plasmas.}

It is well known that high triangularity plasmas have better confinement than corresponding low triangularity plasmas because of higher pedestal density $[43,131]$. In high triangularity, the peeling-ballooning boundary is strongly shaped so at high $j_{b s}$ the pedestal can reach a region with improved pedestal stability (see, for example, [132]).

On the other hand, the first JET-ILW results did not show any clear confinement improvement in the baseline high triangularity plasmas $[17,18,27]$. The hypothesis proposed to explain this behavior [27] was that the baseline pulses were not able to reach a collisionality low enough to reach the strongly shaped region of the PB stability diagram. So far, this hypothesis has been verified only for the hybrid scenario at 1.4MA, in a recent work [83]. This subsection tests if this hypothesis is consistent also with pulses in the baseline scenario (for definitions of hybrids and baseline scenarios, please see references [133, 134]).

Figure 23 shows the correlation between the normalized pressure gradient and the pedestal collisionality. The figure includes both low triangularity $(\delta<0.3$, grey circles $)$ and high 
triangularity plasmas ( $\delta>0.3$, grey triangles). Four subsets are highlighted, two hybrids subsets (at 1.4MA with low and high triangularity) and two baselines (at 2.0MA with low and high triangularity). The high- $\delta$ subsets show a much stronger increase of $\alpha_{\text {exp }}$ with decreasing collisionality compared to the low- $\delta$ subsets. The difference between high- and low- $\delta$ is minimal at high pedestal collisionality $\left(v^{*}>1\right)$ while it is evident at low collisionality $\left(v^{*}<\right.$ 1). This suggests that the nose in the PB stability diagram occurs at $v^{*} \approx 1$. Note the high- $\delta$ pulses have a very similar trend, regardless of the scenario. This result strengthens the hypothesis proposed in [27] and extends it to baseline plasmas and it is consistent with what described in reference [135]. Once the JET-ILW high- $\delta$ baseline pulses reach low collisionality, for example by reducing the gas fuelling rate, a confinement higher than the low- $\delta$ pulses can be reached.

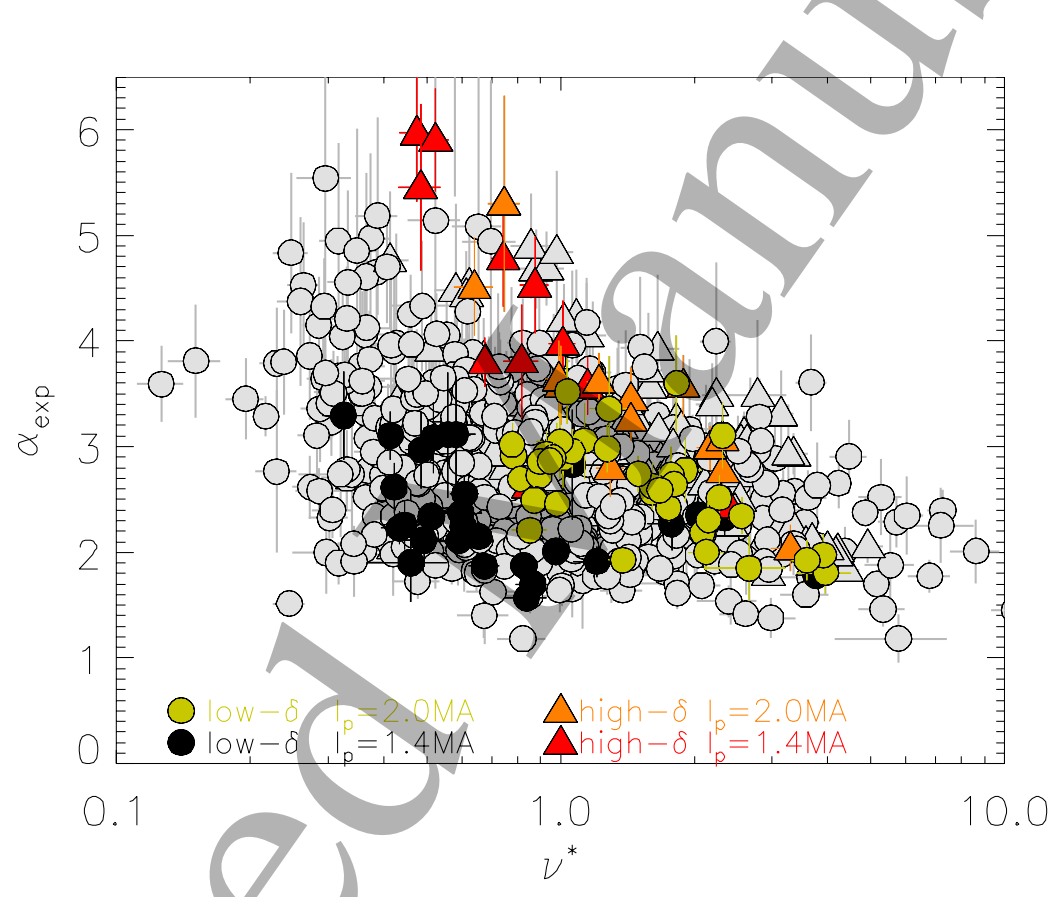

Figure 23. (a) Correlation between the normalized pressure gradient and the normalized electron-ion pedestal collisionality. Grey data show the entire database, separated in low triangularity pulses $(\delta<0.3$,) and high triangularity pulses $(\delta>0.3$,). The non-grey data highlight four subsets: two subsets of hybrid pulses at 1.4MA (low and high $\delta$ ) and two subsets of baseline pulses at 2MA (low and high $\delta$ ).

This subsection can be summarized with the following main results:

- At high collisionality $\left(v^{*}>1\right)$, no difference in the experimental normalized pressure gradient is observed between high and low triangularity plasmas.

- At low collisionality $\left(v^{*}<1\right)$, high triangularity plasmas have $\alpha_{\text {exp }}$ higher than low triangularity plasmas. 


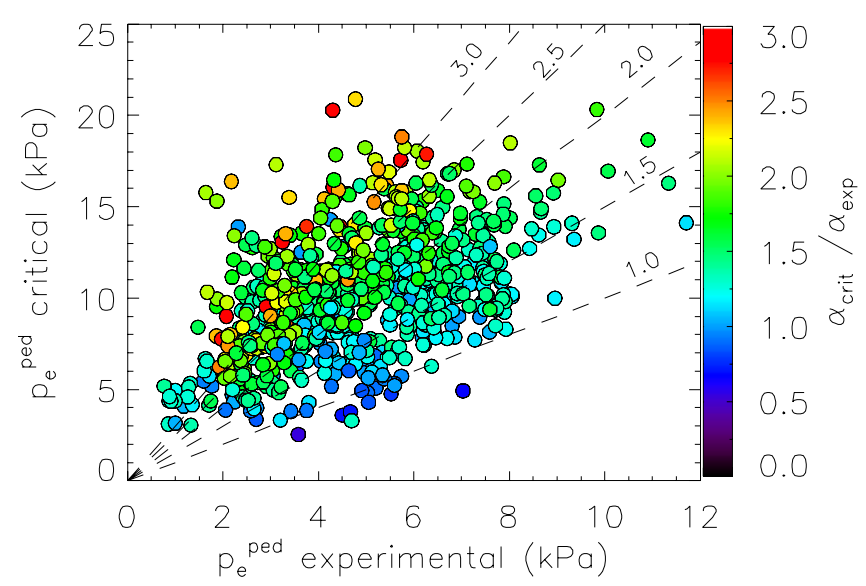

Figure 24. Correlation between the predicted pedestal pressure and the experimental pedestal pressure for the low- $\delta$ pulses in the database. The colors highlight the ratio $\alpha_{\text {crit }} / \alpha_{\text {exp }}$.
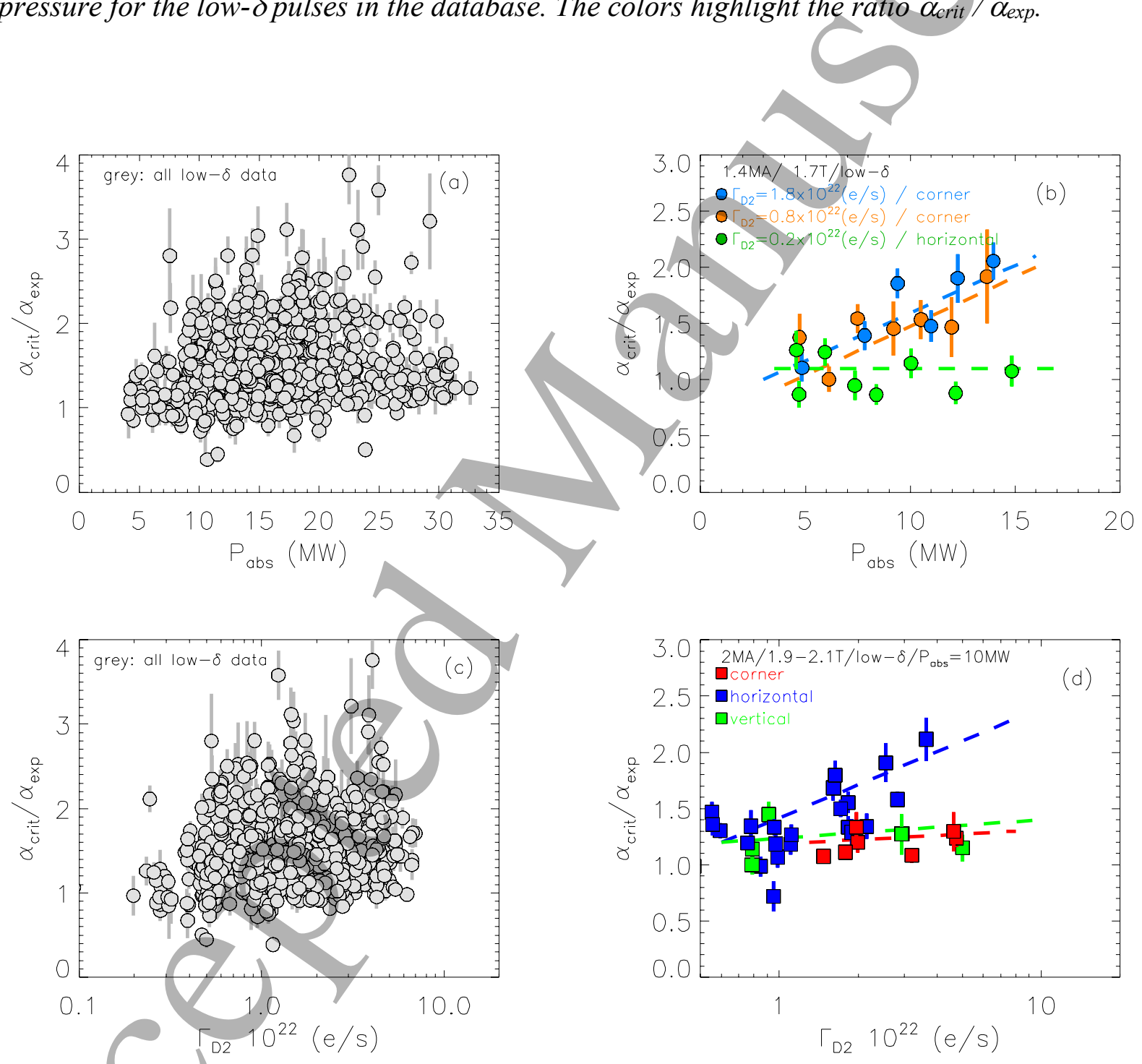

Figure 25. Correlation between the distance of the pre-ELM pedestal from the PB boundary (estimated with the ratio $\alpha_{c r i t} / \alpha_{\text {exp }}$ ) and absorbed power for (a) all low- $\delta$ pulses included in the database and (b) for the same subset of 1.4MA pulses discussed in figures 8 and 9. Correlation between $\alpha_{c r i t} / \alpha_{\text {exp }}$ with fueling rate for (c) the low- $\delta$ pulses in the database and (d) for the same subset of 2MA pulses discussed in figures 10,11 and 12. 


\subsection{Distance from the PB boundary.}

JET-ILW has shown that, even in Type I ELMy H-modes, not all the plasmas reach the PB boundary before the ELM is triggered $[18,27,53]$. This can affect the prediction capability of EPED, as discussed in detail for JET-ILW in reference [66]. In the EPED1 model, the pedestal height is determined by the combination of the PB stability and the KBM limit (which is implemented via the expression $\left.w_{p}=0.076\left(\beta \beta^{\text {ed }}\right)^{1 / 2}\right)$. On the other hand, the JET-ILW pedestal has often $\alpha_{c r i t} / \alpha_{\text {exp }} \gg>1$ while pedestal width is often significantly wider than the predicted EPED1 width (see figure 13 and reference [66]). However, the two effects partially cancel each other, with the lower PB stability compensated by the wider width. As a consequence, EPED1 produces a pedestal pressure still roughly in agreement with the JET experimental pressure. On average, the agreement is within $20 \%$, even if larger deviation have been observed (see figure 2 in reference [66]). The problem is more evident if the $\mathrm{p}^{\text {ped }}$ prediction relies only on the PB stability while the experimental pedestal width is used as input. This is shown in figure 24, where the predicted pedestal pressure is determined using the approach described in section 2.3. The predicted $p_{\mathrm{e}}^{\text {ped }}$ can be up to a factor three overestimated, with the disagreement that increases with increasing $\alpha_{\text {crit }} / \alpha_{\text {exp }}$. Therefore, reliable pedestal predictions in JET-ILW can be achieved only after the physics mechanisms that lead to $\alpha_{c r i t} / \alpha_{\exp }>1$ have been identified.

At present, it is not clear what are the experimental conditions and the physics reasons under which the pre-ELM pedestal is far from the PB/boundary. The only results available so far, based on 1.4MA pulses, suggest that the pedestal is not PB limited at high gas and high power [27].

This subsection investigates in detail the conditions under which the pedestal is not PB limited, shows that a universal threshold in power and/or gas dosing cannot be found and identifies the the relative shift as a key empirical parameter.

\subsubsection{Correlation between $\alpha_{c r i t} / \alpha_{\text {exp }}$ with power, gas dosing rate, divertor configuration.}

The first step is to verify if the main engineering parameters are correlated with $\alpha_{c r i t} / \alpha_{\text {exp }}$. We have considered input power, gas dosing rate, plasma current, triangularity, $q_{95}$ and divertor configuration. $\alpha_{c r i t} / \alpha_{\exp }$ has no obvious correlation with triangularity and $q 95$, even when specific subsets were considered. Triangularity and $q_{95}$ will not be discussed further. On the other hand, trends with power and dosing rate are present for specific subsets as shown in figure 25. Figure 25(a) shows the correlation between $\alpha_{c r i t} / \alpha_{\text {exp }}$ and $P_{a b s}$ for the entire low- $\delta$ database, while figure $25(\mathrm{~b})$ highlights the 1.4MA subset discussed in figure $8(\mathrm{~d})$. The subsets of figure 
25(b) show contradicting results. At low gas (green data), $\alpha_{c r i t} / \alpha_{\text {exp }} \approx 1$ regardless of the power. At higher gas (orange and light blue), $\alpha_{c r i t} / \alpha_{\text {exp }}$ has a positive correlation with the power and the pedestal is near the PB boundary only with low $P_{a b s .}$ This is indeed consistent with the statement that the pedestal is not PB limited at high gas and high power.

However, the extension of the analysis to other subsets shows a more complex behavior. An example is shown in figure 25(d), where the highlighted datasets correspond to the 2MA pulses discussed in figures 10-12 (similar $I_{p}, B_{t}, P_{a b s}$ but different fuelling rate and diyertor configuration). Figure 25(d) shows that also the divertor configuration plays a role. For example, pulses with $\Gamma_{D 2}=5 \cdot 10^{22}(\mathrm{e} / \mathrm{s})$ are near the PB boundary in the corner configuration $\left(\alpha_{c r i t} / \alpha_{\text {exp }} \approx 1.1\right)$ and far from the PB boundary in the horizontal configuration $\left(\alpha_{c r i t} / \alpha_{\text {exp }} \approx 2.0\right)$. Once more, this suggests that the gas fuelling rate is not the most appropriate parameter to have a general description of the plasma but that the neutral pressure might be more suitable. Interestingly, no clear correlation between $\alpha_{c r i t} / \alpha_{\exp }$ and the separatrix density (used as proxy for the neutral pressure) has been observed (not shown here, for simplicity).

Since both neutral pressure and power affect the pedestal position (see section 5.4), it is reasonable to put forward the hypothesis that the main plasma parameters that determine $\alpha_{c r i t} / \alpha_{\exp }$ are $n_{e}{ }^{p o s}, T_{e}^{\text {pos }}$ or a combination of the them.

\subsubsection{The correlation between $\alpha_{c r i t} / \alpha_{\exp }$ and the pedestal position.}

To test this hypothesis, figure 26(a) shows the correlation between $\alpha_{c r i t} / \alpha_{\text {exp }}$ and $n_{e}^{p o s}-T_{e}^{p o s}$ for the subsets of figure 8 . Figure 26(b) shows that same correlation for the subsets of figure 12 and figure 17. In both figures a clear positive correlation between $\alpha_{\text {crit }} / \alpha_{\exp }$ and the relative shift is present. Pedestals near the PB boundary have low relative shift, while pedestal far from the boundary have high relative shift. Note that the pulses on horizontal configuration discussed in figure $8(\mathrm{~g})$ and in figure 9(d) (empty blue circles) have high relative shift and $\alpha_{c r i t} / \alpha_{\text {exp }}>1.3$. Moreover, note that the JET-C subset of figure 12 (black squares) are characterized by low relative shift and $\alpha_{\text {crit }}<\alpha_{\text {exp }} \approx 1$. Even if not shown here, it is worth to mention that correlation with $\alpha_{\text {crit }} / \alpha_{\exp }$ is rather poor when considering $n_{e}^{p o s}$ alone, while it is rather good when considering only $T e^{\text {pos }}$.

At present, there is no theoretical explanation for the correlations shown in figures 26. One of the hypotheses under investigation is that the low normalized pressure gradient is due to an increased turbulent transport driven by the high relative shift [39]. It is beyond the scope of this work to test this hypothesis. Nonetheless, the correlation of figure 26 will be considered in the 
rest of the paper as a useful empirical trend that proves the key role of the relative shift in determining the pedestal performance.

\subsubsection{A more coherent picture.}

At this stage, it is possible to propose a coherent picture that links power, gas fuelling, and divertor configuration with the distance from the PB boundary and that justifies the statement "non-PB limited plasmas are achieved at high gas and power".

The increase of the relative shift is driven by two main parameters, the increase of the power (that leads to the inward shift of the temperature due to the pedestal widening, see figures 14 and 15) and the increase of the neutral pressure (that lead to the outward shift of the density, as shown by the correlation between $n_{e}^{\text {sep }}$ and $n_{e}^{\text {pos }}$ in figure 19). Note that the increase of the neutral pressure can be due to at least two engineering parameters, (i) the increase of the gas fuelling rate and/or (ii) the change to a divertor configuration characterized by less pumping or by a higher fraction of divertor neutrals that reaches the SOL and the pedestal [114]. At low gas dosing rate or in the corner configuration, the neutral pressure is rather low (figure 11) so the pedestal density is located relatively inward and the relative shift cannot reaches very high values even at high power. As consequence, $\alpha_{c r i t} / \alpha_{\exp }$ is relatively close to 1 . At high gas fuelling rate or in the horizontal configuration, the neutral pressure is high and hence the pedestal density is located relatively outward. However, $T_{e}{ }^{p o s}$ is also relatively outward at low power, see figure $15(\mathrm{~b})$, so the relative shift does not reach very high values and $\alpha_{c r i t} / \alpha_{\text {exp }}$ remains close to 1 . The only case in which the relative shift can reach high values (and $\left.\alpha_{c r i t} / \alpha_{\exp }>>1\right)$ is with high $n_{e}^{\text {pos }}$ and low $T_{e}^{\text {pos }}$, so at both high power and high neutral pressure.

Unfortunately, high gas dosing rate and high power are relatively common in JET-ILW. The high gas fuelling is necessary to mitigate the effect of impurity influx from the metal wall. The high power is necessary to compensate the negative effect of the high fuelling on the stored energy.

Note that the JET-C pulses highlighted in figure 26(b) have no gas dosing, low $n_{e}^{\text {sep }}$, pedestal density located inside the separatrix, hence low relative shift, $\alpha_{c r i t} / \alpha_{\text {exp }} \approx 1$ and better pedestal performance than the corresponding JET-ILW pulses (see figures 12, 19 and 25).

\subsubsection{Correlations between $\alpha_{c r i t} / \alpha_{\text {exp }}$ and other plasma parameters.}

Figures 26(a) and 26(b) shows that the relative shift plays a key role in determining the distance from the PB boundary. However, the relative shift might not be the only key parameter or might be simply correlated to the key physics parameters that explains the high $\alpha_{c r i t} / \alpha_{\text {exp }}$ 
ratio. For example, figure 26(b) shows that at $n_{e}{ }^{p o s}-T_{e}^{p o s}=0.02$ the ratio $\alpha_{c r i t} / \alpha_{\text {exp }}$ can vary from 1 to 2, i.e. from PB limited to non-PB limited pedestals, suggesting that also other parameters are important. The rest of this subsection investigates which other plasma parameters might be important in determining $\alpha_{c r i t} / \alpha_{\text {exp }}$ and discusses the corresponding possible implications in terms of physics mechanisms. The following figures will discuss only the parameters that have a correlation with $\alpha_{c r i t} / \alpha_{\text {exp. }}$. Plasma parameters such as separatrix density and collisionality do not show any clear correlation with $\alpha_{c r i t} / \alpha_{\text {exp }}$ and will not be discussed. It is interesting to

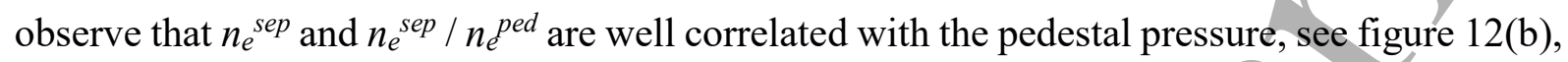
but show no clear correlation with $\alpha_{c r i t} / \alpha_{\text {exp }}$. This is because $\alpha_{c r i t} / \alpha_{\text {exp }}$ increases with increasing relative shift, so it is related to both $n_{e}^{\text {pos }}$ and $T_{e}^{\text {pos }}$, while $n_{e}^{\text {sep }} / n_{e}^{\text {ped }}$ is unrelated to $T_{e}^{p o s}$.

Initially, the subsection will focus on the subsets of figure $8,12,17$. At the end, the same discussion will be extended to the entire database.

\section{Correlation between $\alpha_{\text {crit }} / \alpha_{\text {exp }}$ and $\beta_{N}$.}

An important parameter might be $\beta_{N}$. This is shown in figure 26(c), where the colors highlight the corresponding $\beta_{N}$ of each data point. For similar relative shift, pulses with high $\beta_{N}$ tend to

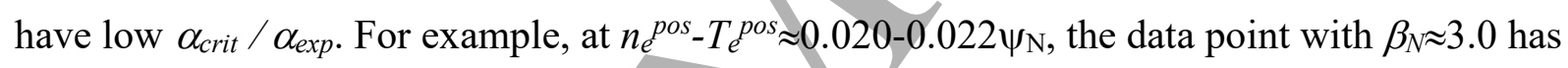
$\alpha_{c r i t} / \alpha_{\text {exp }} \approx 1$, while the data points with $\beta_{N} \approx 1.2$ have $\alpha_{\text {crit }} / \alpha_{\text {exp }} \approx 2$. The empirical correlation between $\alpha_{c r i t} / \alpha_{\text {exp }}$ and $\beta_{N}$, despite having a large scatter, was also observed in reference [66]. This might suggest a stabilizing effect on the micro-instabilities due to the increased $\beta_{N}$ and could explain the low pressure gradient in pulses with $\alpha_{c r i t} / \alpha_{\text {exp }}>1$, but does not help in understanding the ELM triggering mechanism.

\section{Correlation between $\alpha_{\text {crit }}<\alpha_{\text {exp }}$ and resistivity.}

Another parameter that might play a role is the plasma resistivity. Figure 27(a) shows the correlation between $\alpha_{c r i t} / \alpha_{\text {exp }}$ and the Spitzer resistivity (calculated as the average just inside the separatrix, in the region $\psi_{N}=0.98-1.00$ ). A positive correlation seems to be present. This could suggest that the ideal MHD is not sufficient to describe the pedestal stability but that resistive MHD is necessary to identify the PB stability boundary. This hypothesis will be tested in the near future in JET-ILW using the non-linear resistive MHD code JOREK [136]. Indeed, earlier JOREK results were able to reasonably predict the JET-ILW pedestal height [137]. 
Moreover, a pure theoretical result obtain with BOUT++ shows an effect of the resistivity on the PB boundary [138].

It should be pointed out that the correlation shown in figure 27(a) is observed only between the middle of the pedestal and the separatrix. The Spitzer resistivity determined further inward positions has no correlation with $\alpha_{c r i t} / \alpha_{\text {exp }}$. Note that the increase of the resistivity between the middle of the pedestal and the separatrix is, at least partially, linked with the increase of the relative shift via the inward shift of $T_{e}^{\text {pos }}$. From a geometrical point of view, an inward shift of $T_{e}{ }^{p o s}$ leads to a reduction in the $T_{e}$ so the an increase in resistivity at a fixed radial position.

This hypothesis can be useful explain both the ELM triggering mechanism and the low pressure gradient.

\section{Correlation between $\alpha_{c r i t} / \alpha_{\text {exp }}$ and $\eta_{e}$.}

Earlier results $[39,73]$ suggests that also $\eta_{e}$ (defined as the ratio between the gradient length of electron density and electron temperature) is correlated with the normalized pressure gradient and $\alpha_{c r i t} / \alpha_{\text {exp }}$. In fact, the increase of the relative shift leads to an increase in $\eta_{e}$, which in turn destabilizes micro-instabilities and increases the turbulent transport in the pedestal $[39,73,75]$. The correlation between $\alpha_{c r i t} / \alpha_{\exp }$ and $\eta_{e}$ is shown in figure 27(b). $\eta_{e}$ has been calculated at $T_{e}{ }^{p o s}$. The data of figure 26(b) seems to suggest a positive correlation. This result would be consistent with the hypothesis of the increased turbulent transport in the pedestal region. However, we should highlight that the correlation shown in figure $27(\mathrm{~b})$ is very weak and it has been observed only with $\eta_{e}$ determined at $\psi_{N}=T_{e}^{p o s}$. No correlations have been observed at other radial position, for example at the pedestal top. Unfortunately, being the ratio between two gradients, $\eta_{e}$ is a parameter experimentally difficult to estimate. The hypothesis the increase turbulent transport with increasing $\eta_{e}$ can be useful to explain the low pressure gradient in pulses with $\alpha_{c r i t} / \alpha_{\text {exp }}>1$, but does not help in understanding the ELM triggering mechanism.

\section{Correlation between $\alpha_{c r i t} / \alpha_{\exp }$ and $R / L_{T e}$.}

A recent theoretical work [139] shows that, in local and linear gyrokinetic analysis with the GS2 code [140], the toroidal and slab ETG modes are very sensitive to the normalized $\underline{\mathrm{T}}_{\mathrm{e}}$ gradient length in the pedestal, $R / L_{T e}$. So, a possible hypothesis is that the increase in $\alpha_{c r i t} / \alpha_{\text {exp }}$ might be due to the reduction in $\alpha_{\exp }$ driven by the increased pedestal turbulent transport produced by an increased $R / L_{T e}$. Figure 27(c) shows the correlation between $\alpha_{c r i t} / \alpha_{\text {exp }}$ and $R / L_{T e}$, with $R / L_{T e}$ determined at the top of the pedestal temperature. The positive correlation is qualitatively consistent with the hypothesis. Of course, further work is necessary for a 
conclusive claim. It is important to observe that the results are strongly dependent on the position at which $R / L_{T e}$ has been determined. No correlation has been observed at the top of the pedestal density, while a weak negative correlation has been observed in the outer region of the pedestal, between $T_{e}^{p o s}$ and the separatrix. This hypothesis can be useful to explain the low pressure gradient but does not help in understanding the ELM triggering mechanism.

\section{Correlations using the entire database.}

The correlations discussed in figures 26 and 27 are based on small subsets of the database. To investigate the robustness of the results, figure 28 shows the same correlations for the entire low- $\delta$ database. Since figure 26(c) suggests that $\alpha_{c r i t} / \alpha_{\text {exp }}$ is well correlated to $\beta_{N}$, figure 28 highlights three subsets with $\beta_{N}=1.0,1.9$ and 2.8. In the two subsets with lowest beta, $\alpha_{c r i t} / \alpha_{\text {exp }}$ is positively correlated with relative shift, resistivity near the separatrix, $\eta_{e}$ in the middle of the pedestal and $R / L_{T e}$ at the pedestal top. In the subset at high $\beta_{N}$, no correlation is observed and the pedestal seems always close to the PB boundary.

The results of figure 28 strengthen the conclusion obtained in figures 26 and 27. The correlations with relative shift and $R / L_{T e}$ are rather robust and have been observed for any subset with medium-low $\beta_{N}$. Instead, the correlations with resistivity and $\eta_{e}$ seems less robust and, even though trends are visible also at other values of $\beta_{N}$, the scatter of the data is rather large.

This subsection can be summarized with the following main results:

- $\alpha_{c r i t} / \alpha_{\text {exp }}$ is affected by at least three engineering parameters, $P_{a b s}$, gas fuelling rate and divertor configuration (which suggest a dependence on the neutral pressure).

- In terms of plasma parameters, $\alpha_{c r i t} / \alpha_{\exp }$ has a clear positive correlation with the relative shift.

- The relative shift is sufficiently high to have $\alpha_{\text {crit }} / \alpha_{\text {exp }}>>1$ mainly at high power and high neutral pressure. This explains the empirical observation that the JET-ILW pedestal is not PB limited at high power and high gas.

- The physics mechanism that leads to $\alpha_{c r i t} / \alpha_{\text {exp }}>>1$ is still unclear. However, empirical correlations between $\alpha_{c r i t} / \alpha_{\text {exp }}$ and the following plasma parameters strengthen some (or lead to new) hypotheses:

- Resistivity. Ideal MHD might not be sufficient to describe the PB stability in pedestals with high relative shifts. Resistive MHD might be necessary.

Relative shift and $\eta \mathbf{e}$ at $T_{e}^{\text {pos }}$. The increase of the relative shift leads to an increase in $\eta e$ and hence in the turbulent transport.

$\boldsymbol{\beta}_{\mathbf{N}}$. The decrease of $\beta_{\mathrm{N}}$ might have a destabilizing effect on microinstabilities 
- $\mathbf{R} / \mathbf{L}_{\text {Te. }}$ The increase of $\mathrm{R} / \mathrm{L}_{\mathrm{Te}}$ might destabilize toroidal and slab ETG modes increasing the turbulent transport. 
3

4

5
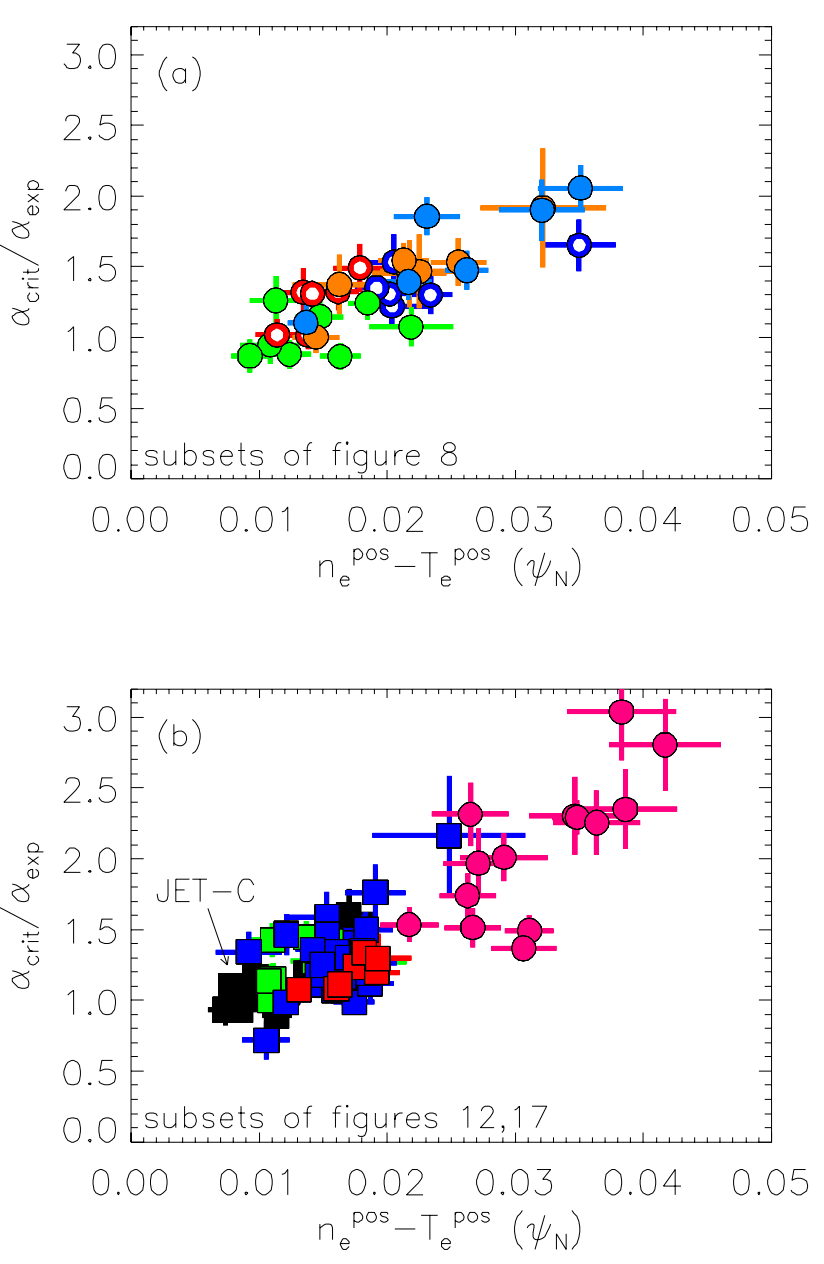

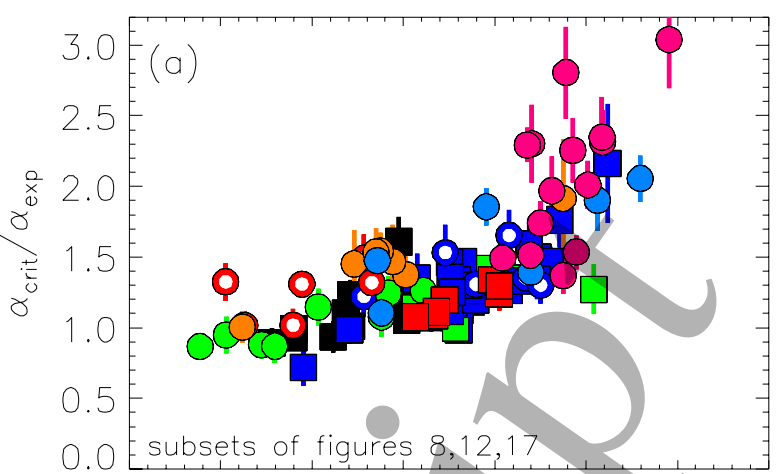

$\begin{array}{lllllllll}3.0 & 3.5 & 4.0 & 4.5 & 5.0 & 5.5 & 6.0 & 6.5\end{array}$ $<\eta_{\text {spitzer }}>$ at $\psi_{N}=0.98-1.0010^{7}(\Omega \mathrm{m})$
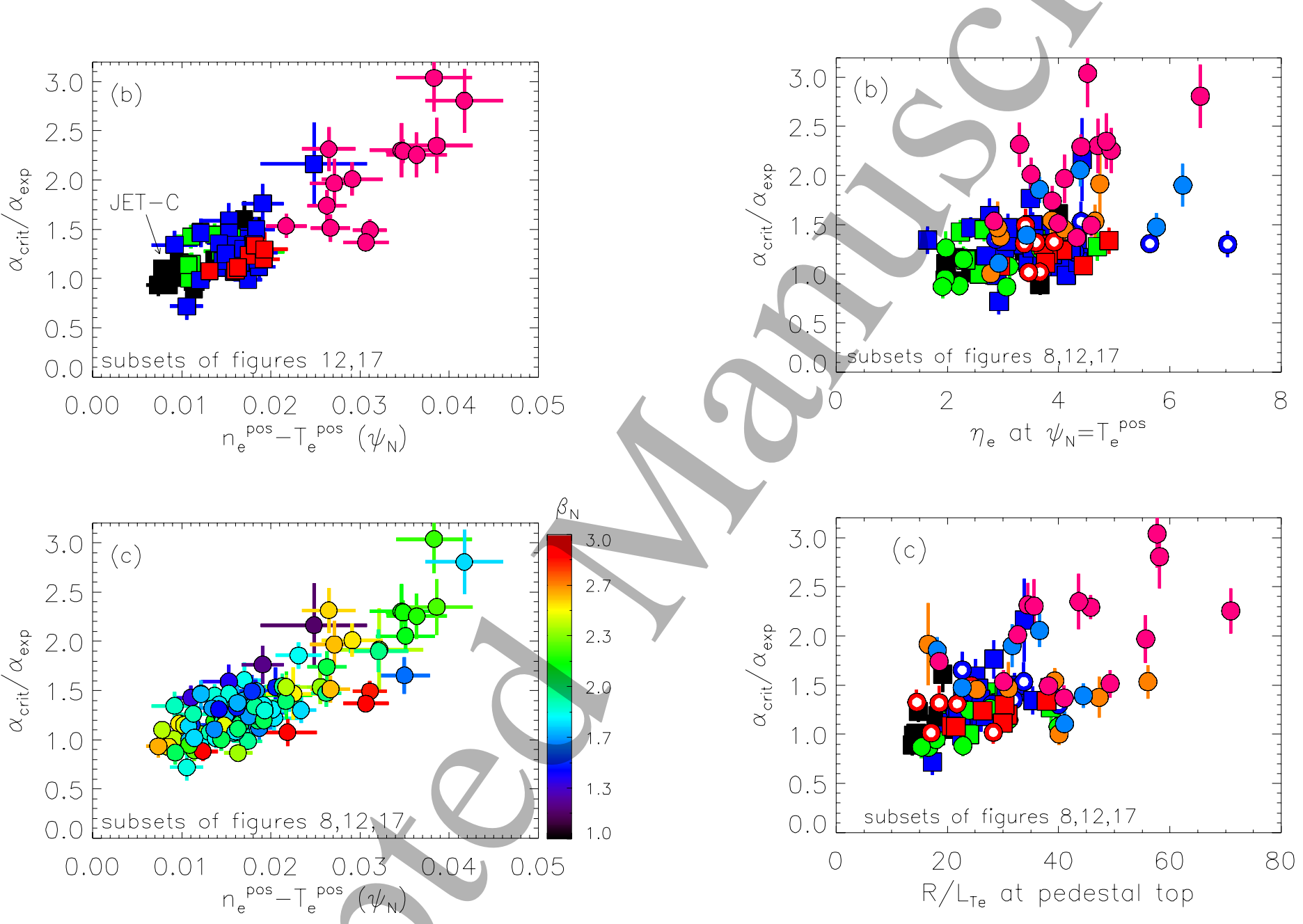

Figure 26. Correlation between $\alpha_{\text {crit }} / \alpha_{\text {exp }}$ with the relative shift for all the subsets used in section 5 . Colors and symbols are consistent with those used in section 5. Frame (a) shows the power scans, frame (b) the gas scans. Frame (c) shows the same data of both frame (a) and frame (b) but with the colors highlighting $\beta_{N}$.

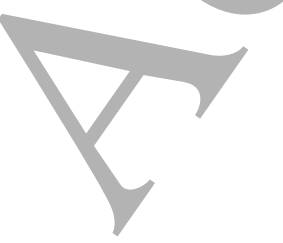

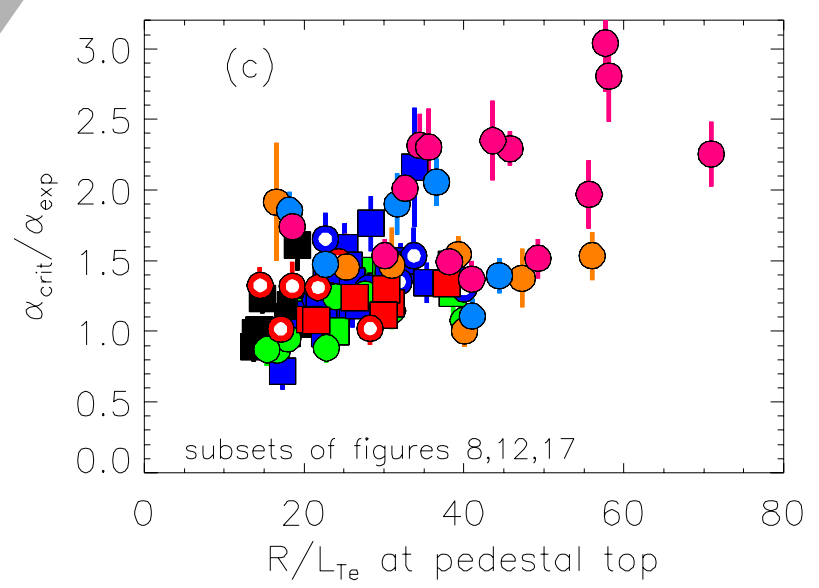

Figure 27. Correlation between $\alpha_{\text {crit }} / \alpha_{\text {exp }}$ with (a) Spitzer resistivity averaged over the radial range $\psi_{N}=0.98-1.00$, (b) $\eta_{e}$ in the middle of the pedestal temperature, (c) $R / L_{T e}$ calculated at the top of the pedestal temperature. Only the subsets discussed in section 5 are shown. Colors and symbols are consistent with those used in section 5. For simplicity, the uncertainty in the horizontal axis is not shown but in frame (b) it is rather large (of the order of $50 \%$ ). 


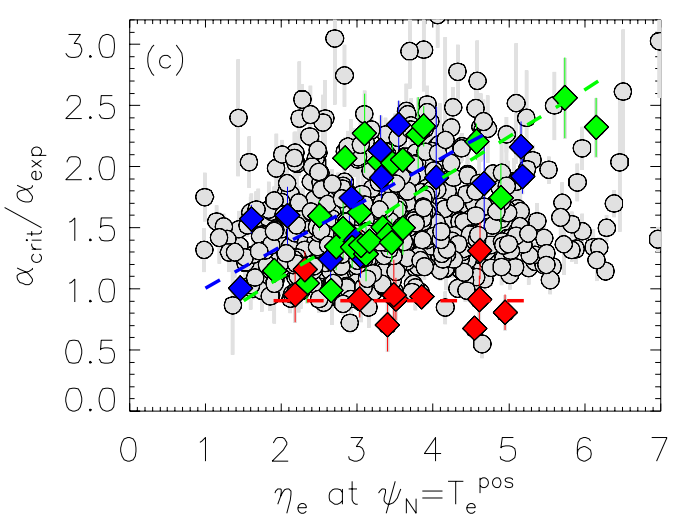

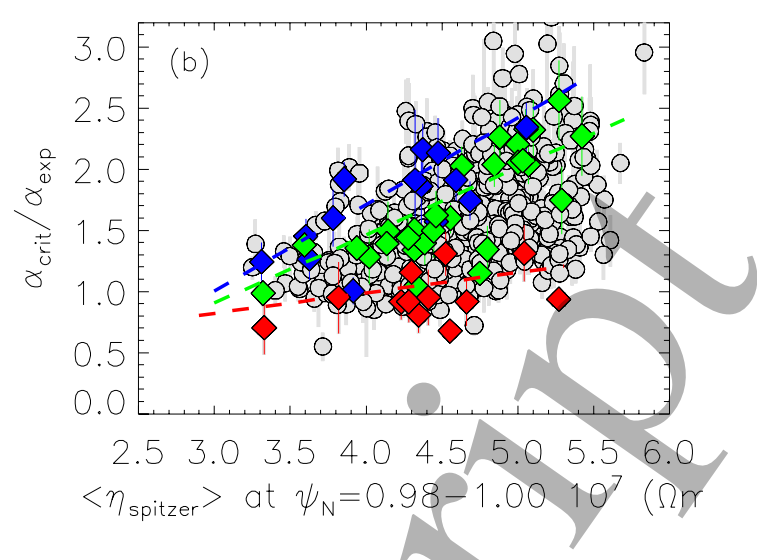

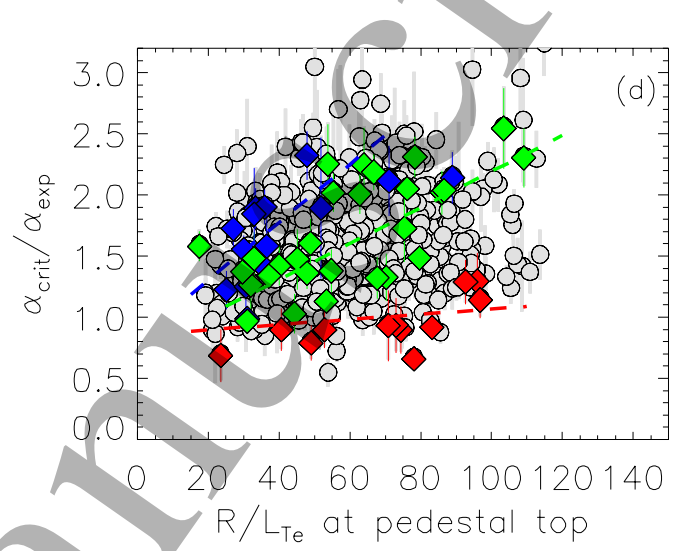

Figure 28. Correlation between $\alpha_{\text {crit }} / \alpha_{\text {exp }}$ with (a) relative shift, (b) Spitzer resistivity averaged over the radial range $\psi_{N}=0.98-1.00$, (c) $\eta_{e}$ in the middle of the pedestal temperature, (d) $R / L_{T e}$ calculated at the top of the pedestal temperature. The grey data shows all the low- $\delta$ pulses of the database. The colors highlight subsets at constant $\beta_{N}$. For simplicity, the uncertainty in the horizontal axis is shown only in frame (a). In frame (c) the uncertainty in the horizontal axis is rather large, (of the order of 50\%). 


\section{PART (C). Pedestal scalings.}

\section{PEDESTAL SCALINGS.}

The results obtained during the first JET-ILW experimental campaigns have shown that baseline plasmas tend to have a pedestal approximately 10-20\% lower than in JET-C [18]. This has cast doubts on the applicability to JET-ILW of the Cordey scaling for the pedestal, which was derived using mainly carbon wall machines [7]. Nowadays, a reliable pedestal scaling for JET-ILW has major importance because of its key role for DT extrapolations [110,111]. As an example, the predictive modelling discussed in $[13,14]$ relies on the pedestal Cordey scaling to determine the boundary condition for the core modelling.

Another important reason to update the pedestal scalings is related to diagnostic quality. As an example, the JET pedestal density used in the Cordey scaling was based on a line-integrated measurements and not on a specific measurement at the pedestal top. Nowadays, the JET Thomson scattering can provide a much more accurate measurement [77].

However, an update of the pedestal scaling úsing the present EUROfusion JET-ILW pedestal database cannot fully replace the Cordey scaling. The geometrical parameters do not vary sufficiently to be included in a scaling. Nonetheless, an updated scaling can still provide valuable information. First of all, it can be used to verify if JET-ILW has a different scaling in terms of power and current from what obtained by Cordey. Second, due to the better diagnostics availability, it can provide a more reliable and robust prediction for JET-ILW DT extrapolations. Third, new important physics parameters can be included in the scaling, such as the separatrix density.

Moreover, the database can be used also to determine scalings for the pre-ELM pedestal density and temperature. In particular, a scaling of the pre-ELM pedestal density is very important for EPED-like predictive runs (which have $n_{e}^{\text {ped }}$ as input) $[28,57,66]$. EPED-like models typically use the experimental $n_{e}^{\text {ped }}$ as input or, more recently, have implemented the neutral penetration model [70] or the Urano scaling [141]. However, the neutral penetration model does not correctly predict the isotope dependence [99] and the Urano scaling has been derived for the line-averaged density.

Unfortunately, some of the engineering parameters included in the database are strongly interdependent. A detailed analysis of the multivariate dependencies among the engineering parameters is outside the scope of this paper. Our analysis is limited to the bivariate linear 
correlations, given by the correlation matrix in Table 2. Specifically, in order to avoid issues due to the strong correlation between magnetic field and plasma current ( $q_{95}$ scans are not common in JET-ILW), the following scaling laws include $I_{p}$, but not $B_{t}$.

Table 2. Correlation matrix among the main engineering parameters: plasma current, magnetic field on axis, triangularity, absorbed power, effective mass, gas fuelling rate.

\begin{tabular}{ccccccc}
\hline & $\mathrm{I}_{\mathrm{p}}$ & $\mathrm{B}_{\mathrm{t}}$ & $\delta$ & $\mathrm{P}_{\mathrm{abs}}$ & $\mathrm{M}_{\mathrm{eff}}$ & $\Gamma_{\mathrm{D} 2}$ \\
\hline $\mathrm{I}_{\mathrm{p}}$ & 1.0 & 0.87 & 0.01 & 0.49 & 0.20 & 0.43 \\
$\mathrm{~B}_{\mathrm{t}}$ & 0.87 & 1.0 & -0.01 & 0.59 & 0.21 & 0.32 \\
$\delta$ & 0.01 & -0.01 & 1.0 & -0.07 & 0.07 & 0.01 \\
$\mathrm{P}_{\mathrm{abs}}$ & 0.49 & 0.59 & -0.07 & 1.0 & 0.16 & 0.14 \\
$\mathrm{M}_{\mathrm{eff}}$ & 0.20 & 0.21 & 0.07 & 0.16 & 1.0 & 0.09 \\
$\Gamma_{\mathrm{D} 2}$ & 0.43 & 0.32 & 0.01 & 0.14 & 0.09 & 1.0 \\
\hline
\end{tabular}

\subsection{Scaling of ELM averaged pedestal stored energy}

A detailed discussion on the definition of ELM-averaged pedestal stored energy has been presented in section 3.2 and in section 3.3. Here, we simply remind that, to have a definition empirically compatible with the one used by Cordey, the stored energy at $\psi_{\mathrm{N}}=0.90$ is used (hereafter called $W_{90}$ ). It has been verified that the exact position is not crucial for the exponents of the scaling law. The exponents do not vary/significantly by changing the position from $\psi_{\mathrm{N}}=0.90$ to $\psi_{\mathrm{N}}=0.94$.

Figure 29(a) compares the experimental $W_{90}$ with the pedestal stored energy expected from the Cordey scaling (equation 1 ). The colours highlight the plasma current. $W_{90}$ and $W_{\text {ped }}^{\text {Cordey }}$ are well correlated. $67 \%$ of pulses have $W_{90} / W_{\text {ped }}^{\text {Cordey }}$ in the range 0.8-1.2 (i.e. the Cordey scaling is consistent within $\pm 20 \%$ with $67 \%$ of the data), see figure 29 (b) and table 3 . However, $W_{90}$ is slightly lower than $W_{\text {ped }}^{\text {Cordey }}$ at high plasma current. Note that the database used in this work has excluded, for simplicity, pulses with N seeding and with pellets. From earlier JETILW studies, it is known that the pedestal pressure can be increased by seeding nitrogen [17] and that the highest plasma performances have been achieved in pulses with pellets [110]. These subsets are shown figure 29(a) as empty symbols. The N seeded pulses (mainly at 2.5MA, high triangularity) significantly exceed the Cordey scaling. The pulses with pellets (mainly at 3.0MA and 3.5MA) achieve a $W_{90}$ roughly consistent with the Cordey scaling. 
The next step in the comparison with the Cordey scaling is to determine the scaling law of $W_{90}$ and compare the exponents. Ideally, the same power law used by Cordey should be implemented. However, due to (i) the correlation between $I_{p}$ and $B_{t}$ and between $I_{p}$ and density (see section 7.2) and (ii) the negligible variation of $R, F_{q}$ and $k_{a}$, only $I p, P$, and $M$ (where $M$ is the isotope mass) can be considered. On the other hand, since also triangularity and fuelling rate affect the pedestal, the new scaling law includes also $\delta$ and $\Gamma_{D 2}$. The dataset used to determine the scaling law is the same as used throughout this work, i.e. excluding plasmas with pellets, seeding, RMPs and kicks (see section 2.4) and considering only type IELMs. However, due to the importance of the isotope mass, hydrogen plasmas have been included. The majority of the hydrogen plasmas is characterized by low $I_{p}$, so their inclusion might adversely influence the fit. To bypass the problem, two scaling laws have been derived, the first one including only deuterium plasmas (hereafter labelled "fit 0 "), the second one including both deuterium and hydrogen plasmas (hereafter labelled "fit 1"). In both cases, a robust Bayesian regression technique has been used, allowing errors in all variables [142]. The results are summarized in expressions (7) and (8) and in table 3:

$$
\begin{aligned}
& W_{90}^{f i t 0}=(0.31 \pm 0.07) I^{1.27 \pm 0.15} P^{0.30 \pm 0.08} \delta^{0.29 \pm 0.12} \Gamma^{-0.07 \pm 0.04} \\
& W_{90}^{f i t 1}=(0.23 \pm 0.07) I^{1.26 \pm 0.15} P^{0.31 \pm 0.08} \delta^{0.29 \pm 0.12} \Gamma^{-0.07 \pm 0.04} M^{0.5 \pm 0.2}
\end{aligned}
$$

where $W_{90}$ is expressed in MW, $I_{p}$ in MA, $P$ is the loss power in MW and $\Gamma$ is in $10^{22} \mathrm{e} / \mathrm{s}$. The inclusion of hydrogen plasmas does not significantly affect the exponents, showing that the result is robust. The error bars on the estimated exponents (posterior means) reflect the range over which the exponents can be varied, taking into account the posterior correlations, such that the median absolute percentage error of the fit increases by a 1-2 percent at most [143]. The error bars defined in this way are typically larger than the usual error estimates based on the standard error (ordinary least squares, OLS) or the posterior standard deviation (Bayesian). Indeed, the traditional error estimates are often unrealistically small, because they depend crucially on the model assumptions (i.e. a power law functional form).

By comparing expression 7 with expression 1, it is possible to note that the $I_{p}$ exponent and the $P$ exponent are slightly lower than those obtained by Cordey $(\alpha=1.41 \pm 0.06$ and $\left.\alpha_{\mathrm{P}}=0.5 \pm 0.04\right)$. The lower $\alpha_{\mathrm{I}}$ is due to the fact that the density is not considered in expressions (7), as discussed later in section 7.2. The exponent of the isotope mass, $\alpha_{M}$, is similar to the one recently determined for the energy confinement in JET-ILW $\left(\alpha_{M}=0.4\right)$ [20] but is significantly larger than that in the IPB98 $(y, 2)$ scaling $\left(\alpha_{M}=0.19\right)$. For JET, this difference has been discussed 
in a recent work on the JET global confinement [144] and it appears to be related to the inclusion of T plasmas. Revisiting the old JET global confinement dataset considering only $\mathrm{H}$ and D plasmas leads to an exponent very close to the present estimate [144]. The positive dependence on the triangularity is very reasonable, as at low collisionality the high- $\delta$ plasmas tends to have higher pedestal performance than low- $\delta$. The extremely weak dependence on $\Gamma_{D 2}$ is likely due to the fact that the gas fuelling rate is not the optimal parameter to estimate the neutral pressure and the actual fuelling (see section 5.2).

\begin{tabular}{|c|c|c|c|c|c|c|c|c|c|}
\hline & $\alpha_{I}$ & $\alpha_{P}$ & $\alpha_{\delta}$ & $\alpha_{\Gamma}$ & $\alpha_{\left(\frac{n^{s e p}}{n^{p e d}}\right)}$ & $\alpha_{\left(\frac{n^{p e d}}{n^{G W}}\right)}$ & $\alpha_{M}$ & $\mathrm{R}^{2}$ & $\pm 20 \%$ \\
\hline$W_{\text {ped }}^{\text {cordey }}$ & $1.41 \pm 0.06$ & $0.5 \pm 0.04$ & - & - & - & & 0.2 & 0.74 & $67 \%$ \\
\hline$W_{90}^{f i t 0}$ & $1.27 \pm 0.15$ & $0.30 \pm 0.08$ & $0.29 \pm 0.12$ & $-0.07 \pm 0.04$ & & & - & 0.89 & $86 \%$ \\
\hline$W_{90}^{f i t 1}$ & $1.26 \pm 0.15$ & $0.31 \pm 0.08$ & $0.29 \pm 0.11$ & 4 & & - & $0.5 \pm 0.2$ & 0.90 & $84 \%$ \\
\hline$W_{90}^{f i t 2}$ & $1.14 \pm 0.13$ & $0.30 \pm 0.08$ & $0.32 \pm 0.12$ & & & - & $0.5 \pm 0.2$ & 0.90 & $88 \%$ \\
\hline$W_{90}^{f i t 3}$ & $1.11 \pm 0.15$ & $0.36 \pm 0.10$ & $0.27 \pm 0.15$ & & & $-0.08 \pm 0.15$ & $0.5 \pm 0.3$ & 0.89 & $81 \%$ \\
\hline$n_{e}^{p e d(f i t 1)}$ & $1.24 \pm 0.19$ & $-0.34 \pm 0.11$ & & & - & - & $0.2 \pm 0.2$ & 0.80 & $78 \%$ \\
\hline$n_{e}^{p e d(f i t 2)}$ & $1.40 \pm 0.18$ & $-0.34 \pm 0.11$ & & & $0.03 \pm 0.03$ & - & $0.2 \pm 0.3$ & 0.78 & $75 \%$ \\
\hline$T_{e}^{p e d(f i t 1)}$ & $0.00 \pm 0.2$ & $0.74 \pm 0.12$ & & $-0.16 \pm 0.05$ & - & - & $0.3 \pm 0.4$ & 0.70 & $64 \%$ \\
\hline$T_{e}^{p e d(f i t 2)}$ & $-0.3 \pm 0.2$ & $0.77 \pm 0.14$ & 9 & - & $-0.11 \pm 0.09$ & - & $0.3 \pm 0.4$ & 0.61 & $69 \%$ \\
\hline$T_{e}^{p e d(f i t 3)}$ & $0.08 \pm 0.19$ & 0.4 & $0.25 \pm 0.16$ & - & - & $-0.87 \pm 0.17$ & $0.5 \pm 0.4$ & 0.76 & $77 \%$ \\
\hline$n_{e}^{\operatorname{sep}(f i t 1)}$ & $1.1 \pm 0.3$ & $-0.43 \pm 0.16$ & $0.82 \pm 0.27$ & $0.23 \pm 0.07$ & - & - & $0.0 \pm 0.3$ & 0.64 & $58 \%$ \\
\hline
\end{tabular}

Table 3. Exponents of the scaling laws for plasma current $\left(\alpha_{\mathrm{I}}\right)$, loss power $\left(\alpha_{P}\right)$, triangularity $\left(\alpha_{\delta}\right)$, gas fuelling rate $\left(\alpha_{\Gamma}\right)$, separatrix density normalized to the pedestal density $\left(\alpha_{(n s e p / n p e d)}\right)$, pedestal Greenwal fraction $\left(\alpha_{(n p e d / n G W)}\right)$ and isotope mass $\left(\alpha_{M}\right)$. The last two columns show the $R^{2}$ and the percentage of the experimental data in the database that are predicted correctly by the scaling law within $\pm 20 \%$. The label "fito" refers to scaling done using only D plasmas. The labels "fit 1 " refer to the scaling done using $D$ and H plasmas and including the gas fuelling rate in the scaling law. The labels "fit 2" refer to the scaling done using D and H plasma and including the ratio $n_{e}^{\text {sep }} / n_{e}^{\text {ped }}$ in the scaling law, while "fit 3 " the ratio $n_{e}^{\text {ped }} / n_{e}^{G W}$. 
Figures 29(c) - 29(f) compare the experimental $W_{90}$ with the two scaling laws in expressions (7) and (8). In both cases, the scaling laws predicts the experimental $W_{90}$ rather well, with $R^{2}=0.89$ for the deuterium dataset (fit 0 ), and $R^{2}=0.90$ for the deuterium and hydrogen dataset (fit 1). The number of experimental data that agree within $\pm 20 \%$ with the scaling laws are $86 \%$ and $84 \%$ respectively. This level of agreement is comparable to that obtained using the EPED model, as discussed for example in [66].

To overcome the problem that the gas fuelling rate is not an optimal parameter to use, a third scaling law has been determined by replacing $\Gamma_{D 2}$ with $n_{e}{ }^{\text {sep }} / n_{e}{ }^{\text {ped }}$. Unfortunately, $n_{e}{ }^{\text {sep }}$ cannot be directly used because it is strongly correlated with the plasma current (see later, in expression 14). $n_{e}^{\text {sep }} / n_{e}^{\text {ped }}$ is not an engineering parameter, but it might be estimated with SOL transport modelling. The use of $n_{e}^{\text {sep }} / n_{e}^{\text {ped }}$ is also more relevant than $\Gamma_{D 2}$ for future comparison with other machines. The scaling law is shown in expression (9):

$W_{90}^{f i t 2}=(0.21 \pm 0.07) I^{1.14 \pm 0.13} P^{0.30 \pm 0.08} \delta^{0.32 \pm 0.12} M^{0.5 \pm 0.2}\left(n_{e}^{\text {sep }} / n_{e}^{\text {ped }}\right)^{-0.23 \pm 0.11}$

The dependence on $n_{e}^{\text {sep }} / n_{e}^{\text {ped }}$ is clearly stronger than the dependence on the fuelling rate. According to the scaling, a $50 \%$ reduction in $n_{e}{ }^{\text {sep }} / n_{e}{ }^{\text {ped }}$ (consistent with the variation of $n_{e}{ }^{\text {sep }}$ due to divertor configuration, as shown in figure 11) leads to a $17 \%$ increase in the pedestal store energy. The uncertainty in the exponent is rather large because the database is weakly populated at low $n_{e}{ }^{\text {sep }} / n_{e} e^{\text {ped }}$. Figures 29(g) and 29(h) compare expression (9) with the experimental data. The use of $n_{e}^{\text {sep }} / n_{e}^{\text {ped }}$ slightly improves the agreement, with $88 \%$ of the experimental data that agree within $\pm 20 \%$ with the scaling law. However, $R^{2}$ is not significantly affected.

Finally, in the fourth scaling the gas rate has been replaced by the pedestal density, which should better describe the fuelling. Due to the strong correlation between $n_{e}{ }^{\text {ped }}$ and plasma current, see section 7.2, the parameter used is the pedestal Greenwald fraction, $n_{e} e^{p e d} / n_{e}^{G W}$ :

$$
W_{90}^{f i t 3}=(0.21 \pm 0.08) I^{1.11 \pm 0.15} P^{0.36 \pm 0.10} \delta^{0.27 \pm 0.15} M^{0.5 \pm 0.3}\left(n_{e}^{p e d} / n_{e}^{G W}\right)^{-0.08 \pm 0.15}
$$

As can be seen from table 3 , the use of the Greenwald fraction does not affect significantly the scaling of the stored energy. 


\subsection{Scaling of $n_{e}^{p e d}, T_{e}^{p e d}$ and $n_{e}^{\text {sep }}$}

A scaling law for the pre-ELM pedestal height of the density is important for EPED-like models, where the density is an input. A scaling law for the pre-ELM pedestal density using the data included in the present database is given in expression (11), with $n_{e}^{\text {ped }}$ in $10^{19} \mathrm{~m}^{-3}$ units.

$n_{e}^{p e d(f i t 1)}=(9.9 \pm 0.3) I^{1.24 \pm 0.19} P^{-0.34 \pm 0.11} \delta^{0.62 \pm 0.14} \Gamma^{0.08 \pm 0.04} M^{0.2 \pm 0.2}$

The comparison between expression (11) and the experimental density is shown in figures 30 (a) and 30(b). The scaling predicts the pedestal rather well, with $\mathrm{R}^{2}=0.80$ and with $78 \%$ of the experimental data agreeing within $\pm 20 \%$ with the scaling law.

The pedestal density is strongly dependent on plasma current and triangularity, as expected. The weak negative dependence with power is also reasonable, see figure $8(\mathrm{e})$. The weak dependence on gas fuelling rate is likely due to the fact $\Gamma_{D 2}$ is not the optimal parameter to estimate the neutral pressure. It is not possible to substitute $\Gamma_{D 2}$ with the separatrix density, due to the strong correlation between $n_{e}{ }^{\text {sep }}$ and $I_{p}$. The dependence on the isotope mass is not yet fully understood and it is currently under investigation $[99,145]$. Finally, we can note that the almost linear correlation between density and plasma current. This strong correlation is the reason for the difference in exponent $\alpha_{I}$ between expression (7) and the Cordey scaling. Expression (7) cannot include the density dependence, while the Cordey scaling contains both plasma current and density, $W_{\text {ped }}^{\text {cordey }} \sim I_{p}^{1.41} n^{-0.15}$. Introducing expression (11), in the Cordey scaling, we obtain $W_{\text {ped }}^{\text {cordey }} \sim I^{1.41} n^{-0.15} \sim I^{1.41}\left(I^{1.24}\right)^{-0.15} \sim I^{1.22}$, which is consistent with the exponent of expression (7).

For the sake of completeness, expression (12) shows the scaling of the pre-ELM pedestal temperature. The $R^{2}$ is rather low (0.70), only $64 \%$ of the experimental data agree within $\pm 20 \%$ with the scaling law and the scatter of the data is rather large, as shown in figures $30(\mathrm{c})$ and $30(d)$.

$T_{e}^{p e d(f i t 1)}=(0.05 \pm 0.03) I^{0.00 \pm 0.2} P^{0.74 \pm 0.12} \delta^{-0.23 \pm 0.15} \Gamma^{-0.16 \pm 0.05} M^{0.3 \pm 0.4}$

Note the lack of any $I_{p}$ dependence. Likely, this is not a physical result, but reflects the fact that the database contains almost no data characterized by a wide range in $I_{p}$ and with other engineering parameters constant. In particular, high $I_{p}$ plasmas have much higher gas dosing 
than low $I_{p}$ plasmas. As a counter example, the small subset highlighted in figure 7(b) with

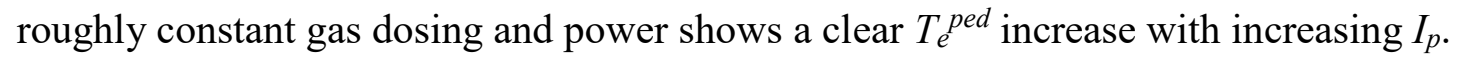

However, as discussed in section 5 , the gas fuelling rate is not an optimal parameter to parameterize the effect of the gas on the plasma, while the separatrix density is more appropriate. Therefore, in expression (13) the fuelling rate has been replaced with the ratio $n_{e}^{\text {sep }}$ $/ n_{e}{ }^{\text {ped }}\left(n_{e}^{\text {sep }}\right.$ alone cannot be used due to its strong correlation with $\left.I_{p}\right)$,

$$
T_{e}^{p e d(f i t 2)}=(0.06 \pm 0.03) I^{-0.3 \pm 0.2} P^{0.77 \pm 0.14} \delta^{-0.15 \pm 0.19}\left(n_{e}^{\text {sep }} / n_{e}^{p e d}\right)^{-0.11 \pm 0.09} M^{0.3 \pm 0.4}
$$

However, this does not lead to a major improvement in the scaling law. The number of experimental data that are predicted correctly within $\pm 20 \%$ increases from $64 \%$ to $69 \%$, but the $R^{2}$ is reduced from 0.70 to 0.61 (see table 2). In expression (14) the fuelling rate has been replaced with the ratio $n_{e}{ }^{s e p} / n_{e}{ }^{G W}$,

$$
T_{e}^{p e d(f i t 3)}=(0.12 \pm 0.05) I^{0.08 \pm 0.19} P^{0.44 \pm 0.12} \delta^{0.25 \pm 0.16}\left(n_{e}^{p e d} / n_{e}^{G W}\right)^{-0.87 \pm 0.17} M^{0.5 \pm 0.4}
$$

The use of the Greenwald fraction in the $T_{e}^{\text {ped }}$ scaling slightly improves the quality of the fit, with $\mathrm{R}^{2}$ increased to 0.76 and $77 \%$ of the data correctly predicted within $\pm 20 \%$. Note that the use of the Greenwald fraction has also change the sign of the triangularity exponent, which is now positive, as in the scaling of the stored energy.

Finally, expression (15) shows the scaling law for the separatrix density:

$$
n_{e}^{s e p(f i t)}=(10 \pm 5) I^{1.1 \pm 0.3} P^{-0.4 \pm 0.2} \delta^{0.8 \pm 0.3} \Gamma^{0.23 \pm 0.07} M^{0.0 \pm 0.3}
$$

The separatrix density has its strongest dependences on the plasma current, with the exponent $\alpha_{\mathrm{l}}=1.1 \pm 0.3$, and triangularity, with $\alpha_{\delta}=0.8 \pm 0.3$. The negative dependence with power $\left(\alpha_{P}=-\right.$ $0.4 \pm 0.2$ ) is consistent with the power scan discussed in figure 9(a), where the pulses with highest power had the highest pedestal temperature and the lowest separatrix density. The positive scaling with fuelling rate is consistent with the results of figure 11(a). Figure 30(e) shows the comparison between the experimental $n_{e}{ }^{\text {sep }}$ and expression (15) (for simplicity, due to the negligible exponent $\alpha_{M}$ in expression 15, only D plasmas are shown). Note the systematic difference between divertor configurations, with plasmas in the corner that tend to have lower $n_{e}{ }^{\text {sep }}$ than plasmas in the horizontal or vertical configuration. This result, which is consistent 
with the discussion of figure 20(c), is particularly evident once pulses with similar predicted $n_{e}{ }^{\text {sep }}$ are considered. This is shown in figure 30(f), where the distribution of the ratio $n_{e}^{\text {sep }}$ $n_{e} e^{\operatorname{sep}(f i t)}$ is shown for the pulses characterized by $2 \times 10^{19} \mathrm{~m}^{-3}<n_{e} e^{\text {sep(fitl) }}<3 \times 10^{19} \mathrm{~m}^{-3}$. Pulses in the corner configuration have the distribution shifted to lower $n_{e}^{\text {sep } /} n_{e}{ }^{\text {sep(fitl) }}$ than pulses in the horizontal or vertical configuration. At present, it is not possible to remove this systematic difference from the scaling. A possibility would be to substitute the gas fuelling rate with the neutral pressure in the divertor, but, as discussed in section 5.2 and figure 11, this is not possible as only neutral pressure measurements in the sub-divertor region are available in JET-ILW.

Ideally, it would be interesting to compare the exponents of expression (15) with analytical models, for example that described in reference [112]. However, a direct comparison is not possible. First of all, the Kallenbach scaling includes the neutral pressure and not the gas fuelling rate. Second, $P_{\text {sep }}$ was used in [112], while $P_{\text {loss }}$ is used in expression (15). Moreover, expression (15) includes the $I_{p}$ dependence, which is not considered directly in the Kallenbach scaling. 

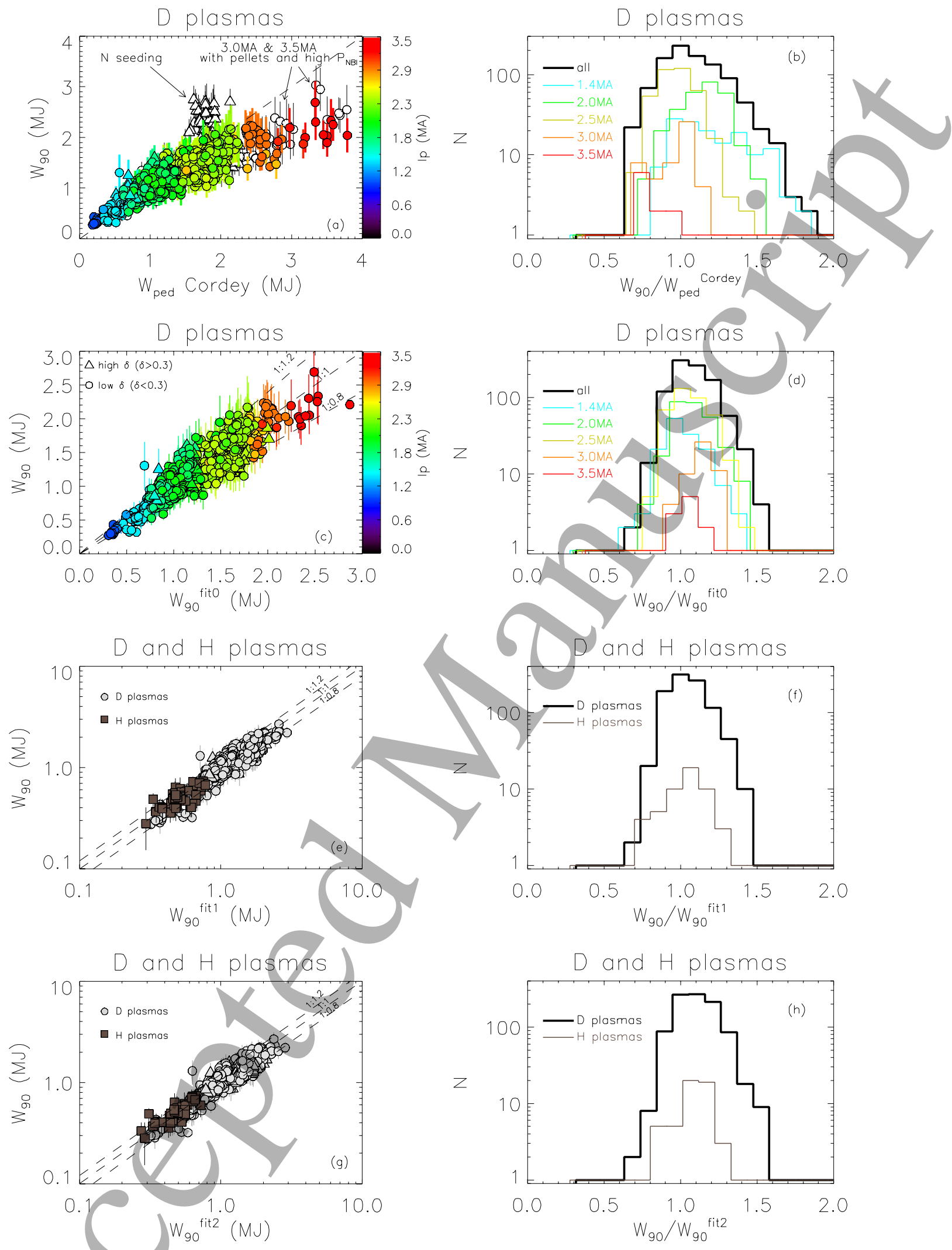

Figure 29. (a) Comparison between the experimental $W_{90}$ and the $W_{\text {ped }}$ from the Cordey scaling for D plasmas. Colors highlight the plasma current. (b) Distribution of $W_{90} / W_{\text {ped }}$ cordey. The colors highlight the distributions for subsets at different $I_{p}$. (c) Correlation between experimental $W_{90}$ and $W_{90}$ from fit 0 for D plasmas (d) Distribution of $W_{90} / W_{90}{ }^{\text {fit }}$. Frames (e) and (f) show deuterium and hydrogen data using fit1. Frames (g) and (h) show deuterium and hydrogen data using fit 2 . 

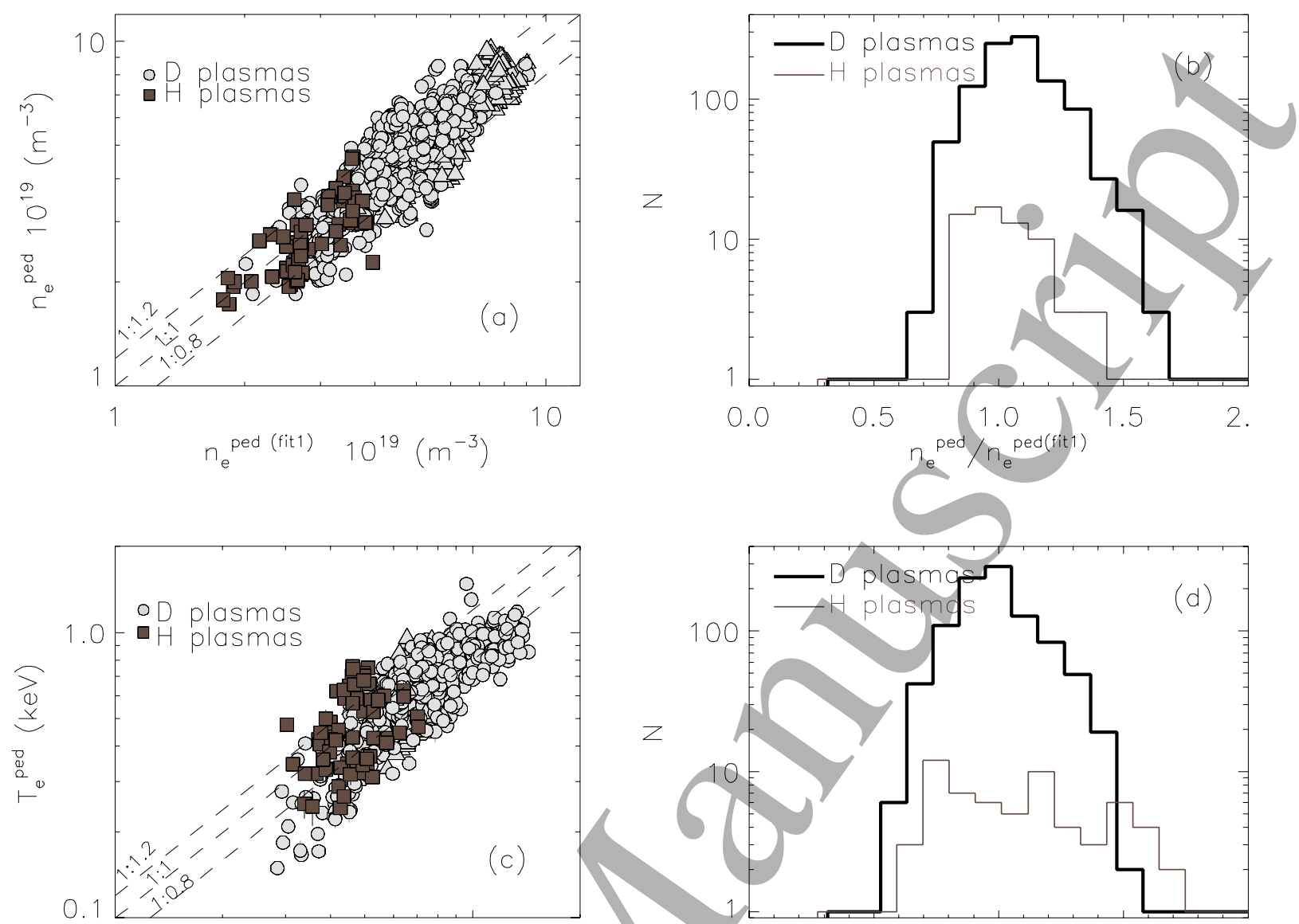

0.1

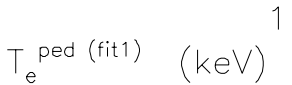

1.0
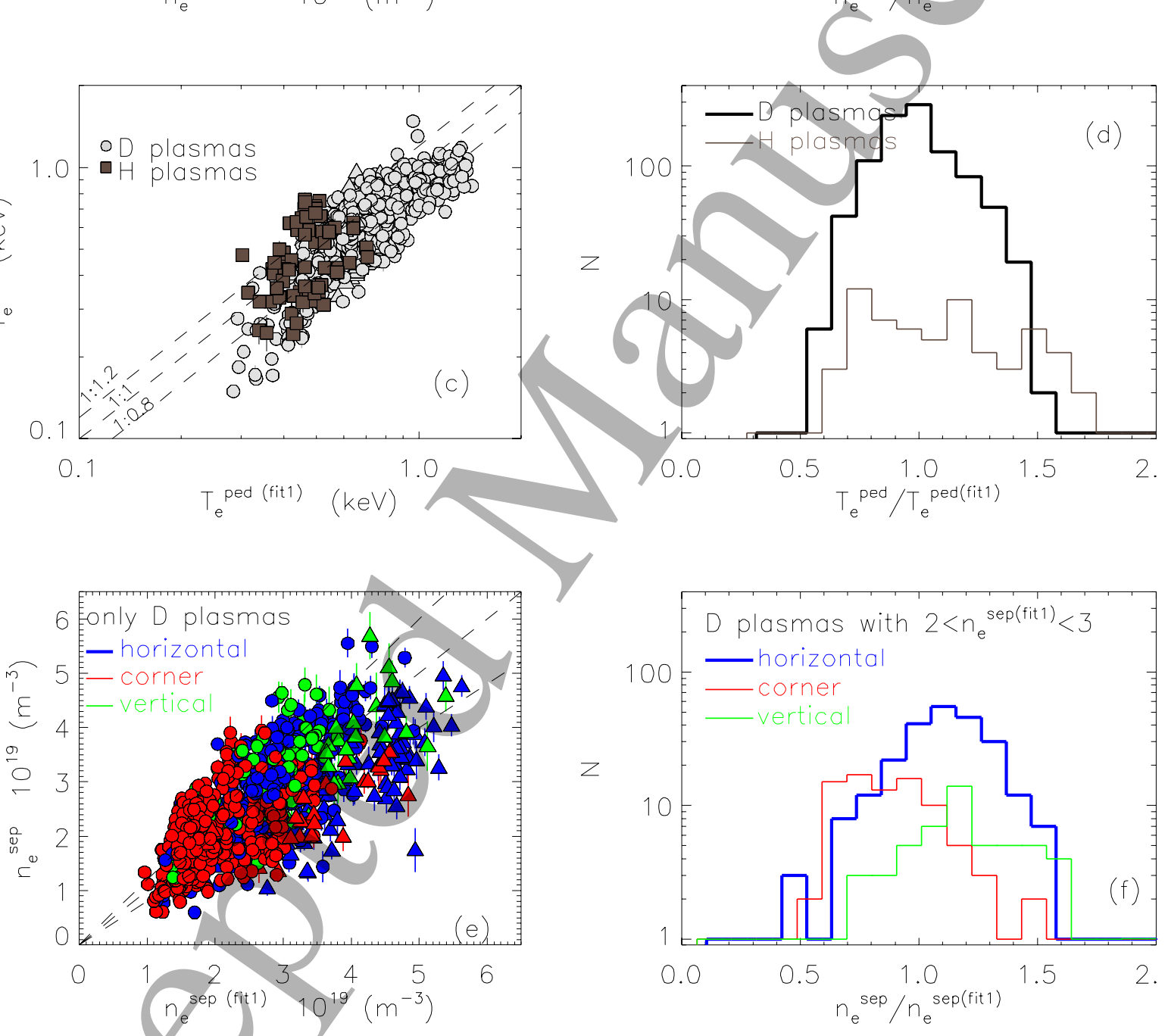

Figure 30. (a) Comparison between the experimental pedestal density and $n_{e}^{\text {ped }}$ from expression (11). (b) Distribution of the ratio between experimental density and density from the scaling law. (c) Comparison between the experimental pedestal temperature and $T_{e}^{\text {ped }}$ from expression (12). (d) Distribution of the ratio between experimental temperature and temperature from the scaling law. (e) Comparison between the experimental separatrix density and expression (15) for D plasmas. (f) Distribution of the ratio between experimental and predicted $n_{e}^{\text {sep }}$ for plasmas with similar separatrix density. 


\section{PART (D). Discussion and conclusions}

\section{DISCUSSION.}

\subsection{Links between engineering parameters and pedestal pressure.}

This work has experimentally investigated under which conditions the JET-ILW type I ELMy H-modes are far from the ideal PB boundary and has shown that the relative shift plays a key role. Ultimately, the origin of the change in the relative shift can be linked to a change in engineering parameters via a rather complex interplay with pedestal width and separatrix density. However, a large number of questions still remain open. Figure 31 summarizes all the logic links experimentally identified in this work (and, for completeness, it includes also previous well known results). The top part figure 31 highlights the changes in the engineering parameters discussed in this work. The bottom part of the figure highlights the effects on the pedestal pressure. The continuous arrows highlight the links for which a theoretical understanding is available. The dashed arrows highlight the links that, at the moment, are based only on empirical correlations. The grey areas highlight the novel results presented in this work.

The extreme left part of figure 31 describes the well known behavior with power. The increasing $P_{a b s}$ leads to an increased core $\beta_{\mathrm{N}}$ which increases the Shafranov shift and hence improve the PB stability and ultimately the pedestal pressure. The increase of the pedestal pressure further increases $\beta_{\mathrm{N}}$ leading to a positive feedback loop.

The top-right part of figure 31 describes the effect of (i) the increase gas fuelling and (ii) the change of divertor configuration (from a configuration with good pumping to one with less pumping). These lead to an increase of the neutral pressure which is experimentally seen an increase in the separatrix density. This link is based on the experimental results discussed in section 5.2 and on EDGE2D-EIRENE simulations of L-mode plasmas [107, 118, 121, 122] and it is rather reasonable. The increase of $n_{e}{ }^{\text {sep }}$ leads to the outward shift of the pedestal density. This link is based on the experimental results discussed in section 5.4 and it will require an in depth investigation with SOL modelling. It is however a reasonable result which is consistent with what observed in AUG [127]. The outward shift of the density can lead to an increase in the relative shift. At this stage, two mechanisms are possible:

(I) If the relative shift remains relatively low, the pedestal is PB limited (see section 6.4). In this case, the outward shift of the density leads to an outward shift of the pressure that 
destabilizes the PB modes, reduces the stability and hence the pedestal pressure, see figure 22 and references [28, 39, 104].

These links explain:

(a) the negative correlation between $p_{e}^{\text {ped }}$ and $n_{e}^{\text {sep }}$ (at low $n_{e}^{\text {sep }}$ ) shown in figure 12(b)

(II) If the relative shift increases to high values $\left(>0.02 \psi_{\mathrm{N}}\right)$, the pedestal is not anymore PB limited (see section 6.4) and the experimental normalized pressure gradient is significantly reduced. The mechanism that leads to this reduction is not understood yet, however the experimental results discussed in section 6.4 suggests some hypothesis. The increase of $n_{e} e^{\text {sep }}$ and of $n_{e} e^{p o s}$ lead to the increase of $\eta_{\mathrm{e}}$, due a simple geometrical effect [39]. In turn, this destabilizes micro-instabilities, hence increasing the turbulent transport $[73,75,76]$ and leading to a reduction of the gradient. The reduction of the gradient (in particular of $T_{e}$ ) leads to the increase of resistivity, making resistive MHD effects non-neglibible, see figure 27(a). A pure theretical results with BOUT ++ indeed suggests that the PB stability is reduced with increasing resistivity [138].

Further theoretical investigation is necessary to validate these links in JET-ILW. Nonetheelss, whatever the physics mechanism is, the increase of the relative shift leads to a marked increase in $\alpha_{c r i t} / \alpha_{\text {exp }}$ (see figure 26) and a strong pressure gradient reduction which in turn produces a significant pedestal degradation. These empirical links explain qualitatively:

(b) the reduction of $p_{e}^{p e d}$ from low to high gas fuelling at constant power $\left[P_{a b s}=15 \mathrm{MW}\right.$ in figure $8(\mathrm{f})]$,

(c) the difference in pedestal performance between divertor configuration in figure 12(a)

(d) the negative correlation between $p_{e}^{\text {ped }}$ and $n_{e}^{\text {sep }}$ (at high $n_{e}^{\text {sep }}$ ) in figure 12(b)

Then, high power and high neutral pressure can also act simultaneously. The increase of the power leads to an increase of the pedestal width, as shown in figure 14(b). The reason for this behavior is unclear but might still be correlated with turbulent transport [146]. The increase of the width leads to an inward shift of the pedestal temperature, figure 19(a), which further increases the relative shift (moreover, the inward shift of $T_{e}$ can further increase the resistivity, amplifying the non-ideal MHD effects). Therefore, high gas / high power operation tend to have a very high relative shift, so the corresponding pedestals tend to be far from the PB boundary. Note that the results discussed in section 6.4 show that a universal threshold in power and gas cannot be found to identify non-PB limited plasmas. This is because any combinations of 
power, gas and divertor configuration that leads to high relative shift produces non-PB limited pedestals. Unfortunately, JET-ILW often operates with high gas fuelling and high power. This explains why a large part of JET-ILW pedestals is not PB limited.

The links described in figure 31 also explain:

(b) the weak or negligible increase of $p_{e}^{\text {ped }}$ with power on the horizontal configuration shown in figure 8(i)

In this case, two mechanisms lead to opposite effects. (1) The increase of the power tends to increase the pressure gradient (see the left side of figure 31). (2) At the same time, the horizontal target operations are characterized by lower pumping, so by higher neutral pressure and higher $n_{e}{ }^{\text {sep }}$, figure 11 . This leads to a significant outward shift of the pedestal density, figure 20 , and hence an increase of the relative shift. Moreover, the increase of the power produces a further increase of the relative shift (via the pedestal widening and the inward shift of $T_{e}^{\text {pos }}$, figure 19). As consequence, the pedestal becomes non-PB limited and the normalized pressure gradient is reduced (perhaps also due to increased turbulent transport, see discussion in section 6.4.4). The two effects tend to cancel each other and, consequently, the increase of $p_{e}{ }^{\text {ped }}$ with power is weak or negligible. 


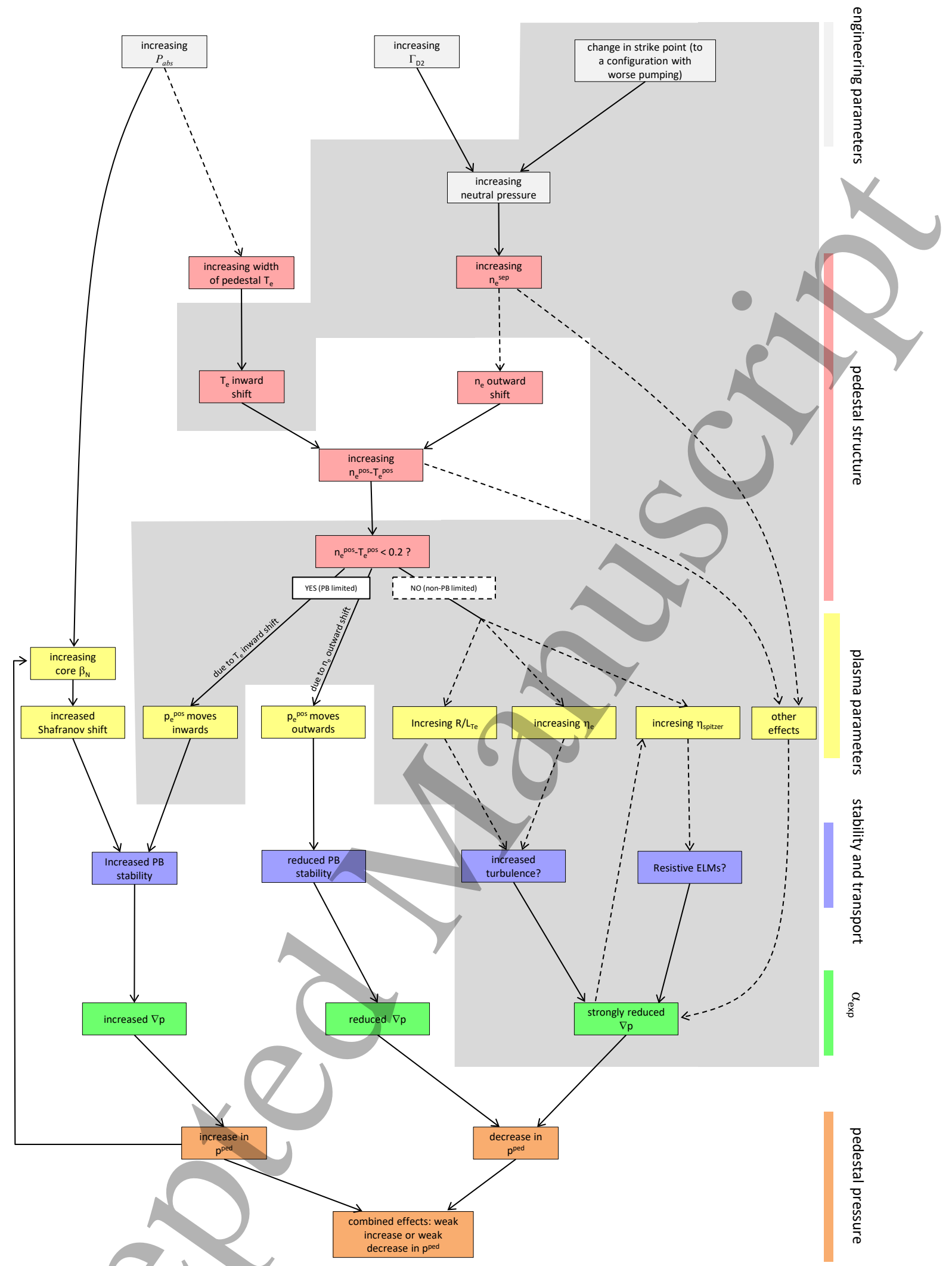

Figure 31. Simplified schematic view of the links that correlate the changes in power, gas fueling rate and divertor configuration to the variation in the pedestal pressure in low- $\delta$ deuterium plasmas. The dashed arrows highlight links empirically observed and that still lack a theoretical explanation. The continuous arrows highlight the links that are fully understood. The grey areas highlight the novel results discussed in this work. For simplicity, some effects (such as the direct effect of the width on the stability and on the pedestal height) are not included. 


\subsection{Some of the effects not discussed in this work.}

The links shown in figure 31 represent a simplified view and some effects have not been included for simplicity. An example is the role of collisionality. The increase of gas fuelling can lead to an increase in the pedestal density, see figure 10, hence to an increase in collisionality. This can lead to a further reduction in the PB stability, see figure 21(b). As another example, the entire work has neglected the effect of impurities, both intrinsic and seeded. The increase of intrinsic impurities can lead to an increased collisionality, via the higher

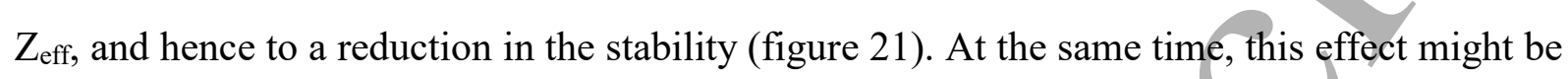
compensated by an increase in the electron temperature due to the dilution effect (see for example [36]). The impurity seeding has also shown a significant effect on the pedestal. Detailed discussions on seeded plasmas can be found in references [17, 26, 32].

Another point not discussed in this work is the role in the PB stability of diamagnetic term, rotation and possibly of $T_{i}^{s e p}>T_{e}{ }^{s e p}$. Unfortunately, systematic measurements of pedestal rotation and $T_{i}^{\text {sep }}$ are not available. However, the inclusion of both terms can reduce the PB stability and move the boundary to a $20 \%-30 \%$ lower $\alpha[32,56]$. Such a difference does not seem sufficient to explain pedestals with $\alpha_{c r i t}\left\langle\alpha_{\exp }>2\right.$, but larger effects cannot be excluded at the moment. Assuming that diamagnetic term and $T_{i}^{\text {sep }} \neq T_{e}^{\text {sep }}$ can explain the pedestal behavior, the empirical correlation with the relative shift would still be present. A possible speculation could be that the increase of the relative shift might be linked with the increase in $T_{i}^{\text {sep }}$ and/or to plasma rotation. The picture shown in figure 31 would not be much altered. A further yellow box is added on the right side that is linked to the increased relative shift and/or the increased $n_{e}^{\text {sep }}$.

\subsection{Discussing the pedestal width and pedestal gradient behavior within the EPED framework}

The pedestal pressure width has been discussed in section 5.3. In particular, figure 13 has shown that the pedestals that are PB limited have a width consistent with the EPED1 assumption $\left(w_{p e}=0.076\left(\beta_{\theta} \theta^{p e d}\right)^{1 / 2}\right)$. In this section, the result of figure 13 is interpreted within EPED1.6 model [58]. This model differs from the standard EPED1 [57] in the assumptions used for the pedestal width. While EPED1 assumes that $w_{p e}=D\left(\beta_{\theta}{ }^{p e d}\right)^{1 / 2}$ with $D=0.076$, the EPED1.6 model assumes that the value of $\mathrm{D}$ is determined by turbulent transport driven by KBMs, so that $D$ can be determined from pulses to pulse. At constant $\beta \theta^{\text {ped }}, D$ is in fact inversely proportional to the 
pressure gradient. So an increase in D would correspond to a reduced pedestal gradient and an increased turbulent transport.
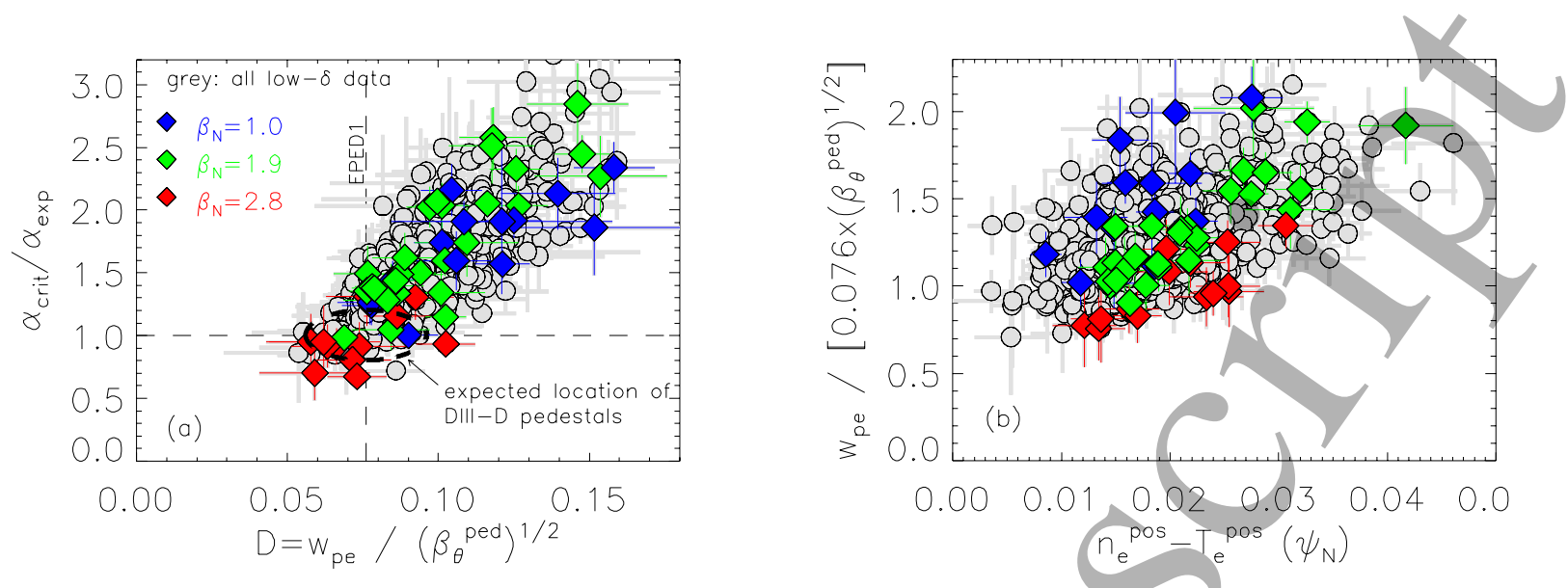

Figure 32. Frame (a). Correlation between $\alpha_{c r i t} / \alpha_{\text {exp }}$ and the parameter D, determined from the expression $w_{p e}=D\left(\beta_{\theta}{ }^{\text {ped }}\right)^{1 / 2}$. The vertical dashed line shows the EPEDI assumption, with $D=0.076$. Frame (b) Ratio of the pedestal width over the width determined from the EPEDI model versus the relative shift. Both frames highlight the same subsets used in in figure 28. In both frames, $w_{p e}$ has been determined using the standard pedestal width definition (average between width of density and temperature).

Figure 32(a) shows the correlation between the distance from the PB boundary and the parameter $D$ for all the low- $\delta$ pulses of the database. The colors highlight subsets with constant $\beta_{N}$ (same subsets of figure 28). For simplicity, the figure shows $D$ estimated with only the standard definition of pedestal width $\left(w_{p e}=\left(w_{T e}+w_{n e}\right) / 2\right)$ (the other two definitions lead to the same conclusions). The positive correlation is very clear. Low $D$ values correspond to pedestal near the PB boundary while high $D$ values to pedestal far from the PB boundary. Within the EPED1.6 framework, this suggests that pedestal far from the PB boundary are characterized by a high level of turbulent transport that reduces significantly the pedestal gradient. This would be consistent with the hypothesis discussed in section 6.4.

An interesting result from figure 32 (a) is related to the value $D=0.076$, i.e. the standard $D$ used in EPED1. JET-ILW pedestal with $D=0.076$ have $\alpha_{\text {crit }} / \alpha_{\text {exp }} \approx 1$ as shown both in figure 13 and in figure 32(a). This is likely not a coincidence and suggests that the correlation in figure 32(a) might be valid for any machines. Since the $D=0.076$ was originally determined from a fit to DIII-D plasmas, which are typically on the PB boundary, it is reasonable to expect that DIII-D pedestals are located near the intersection of the dashed lines, corresponding to $D=0.076$ and $\alpha_{\text {crit }} / \alpha_{\text {exp }}=1$. 
Another important information of figure 32(a) is that the EPED1 assumption for the pedestal width does not describe the experimental JET-ILW $w_{p e}$ only when $\alpha_{c r i t} / \alpha_{\exp }>1$. In fact, the ratio of the experimental $w_{p e}$ to the EPED1 $w_{p e}$ is $w_{p e}^{\text {exp }} / w_{p e}^{E P E D 1}=D^{\exp } / 0.076$. Due to the clear positive correlation between $\alpha_{c r i t} / \alpha_{\exp }$ and $D$ in figure $32(\mathrm{a})$, we can conclude that the experimental conditions that lead to $\alpha_{c r i t} / \alpha_{e x p}>1$ are the same that lead to $w_{p e}^{e x p} / w_{p e}^{E P E D 1}>1$. So, the discussion in section 6.4 on $\alpha_{c r i t} / \alpha_{\exp }$ can be extended to $w_{p e}^{\exp } / w_{p e}^{E P E D 1}$. As an example, figure 32(b) shows the correlation between $w_{p e}^{e x p} / w_{p e}^{E P E D 1}$ with the relative shift. The result is similar to that described in figure 28(a). For similar values of $\beta_{\mathrm{N}}$, the disagreement between the experimental width and the EPED1 width increases with increasing relative shift.

It is important to observe that an increased transport can lead to an increase in the pedestal width above the EPED1 predictions and a reduction of the pressure gradient. However, this does not necessarily imply a pedestal pressure lower than the predicted EPED1 pressure. For example, references $[126,147]$ shows that an increased transport leads to both a wider pedestal width and a higher pedestal height. This can be understood from the schematic illustrations in reference [147] and in figure 33. The EPED prediction assumes that the pedestal width and height are determined by the PB stability and KBM limit, see the intersection of the red and blue continuous lines in figure 33. Assuming no change in the PB stability, an increased transport would modify the KBM constraint (see the light blue dashed line) leading to a wider width but also to a higher pedestal. The increased transport alone would not be sufficient to explain the lower pedestal height observed in some JET-ILW pulses. This can be obtained only if also the PB stability is reduced, see the orange dashed line in figure 33. The experimental results discussed in section 6.4 suggest that in JET-ILW the increased transport might be due to the increased relative shift (which lead to an increased $\eta_{\mathrm{e}}$ ), while the reduced stability might be due to the higher resistivity which makes non-ideal MHD effects non-negligible. 


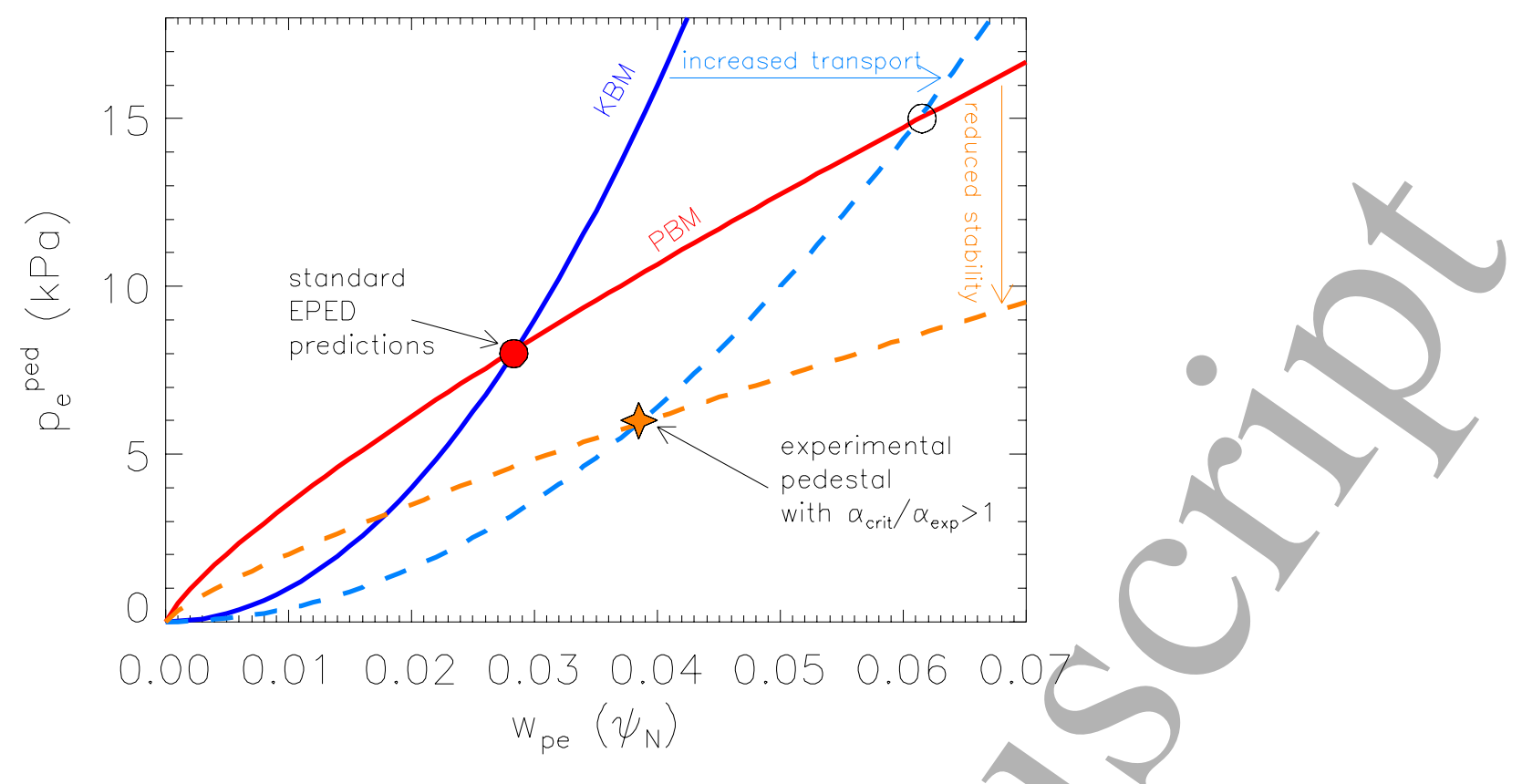

Figure 33. Schematic illustration of the effect of increased pedestal transport and reduced PB stability on EPED-like pedestal predictions.

\section{CONCLUSIONS.}

This work has described the EUROfusion JET-ILW database. Three main aspects of the database have been discussed, (i) the technical aspects, (ii) the pedestal structure and stability and (iii) the pedestal scaling.

The first part of the work has presented the technical aspects, for future reference. These include a description of the selection criteria, datasets, diagnostics and method used, both experimental and theoretical, and main definitions.

The second part of the work has discussed the pedestal structure in JET-ILW and has described its pedestal stability. In particular, the work has tried to shed light into the statements that "the JET-ILW does not reach the PB boundary in plasmas characterised by high gas fuelling rate and high power" [27]. The open issue of this statement is that, so far, clear thresholds in gas fuelling and power have not been observed. The work has shown that the links between engineering parameters (gas fuelling, power but also divertor configuration) and pedestal stability is rather complex and it is due to the interplay between pedestal width and neutral pressure with the pedestal position. The increase of the power leads to a widening of the pedestal temperature which in turn leads to an inward shift of the pedestal position. The increase of the gas fuelling and/or the change to a divertor configuration with less pumping lead to an increase of the neutral pressure and of the separatrix density which produces an effective outward shift of the density position. Both effects lead to the increase of the relative shift. As shown in figure 
26 , the increase of the relative shift is correlated to the increase of $\alpha_{c r i t} / \alpha_{\text {exp }}$. This explains why a universal threshold on gas and power cannot be found: any combinations of power, gas and divertor configuration that leads to high relative shift produces non-PB limited pedestals. Unfortunately, due to operational constraints JET-ILW often operates with high gas fuelling rate and high power [19], so pedestals with $\alpha_{c r i t} / \alpha_{\text {exp }}>1$ are rather common. The work has also highlighted that the EPED1 model works well when $\alpha_{c r i t} / \alpha_{e x p}=1$, as the EPED1 predictions for both gradient and width are in good agreement with the experimental ones.

In the third part of the work, the pedestal scaling has been described. In particular, the scaling of the JET-ILW pedestal stored energy (determined as the stored energy at $\psi_{\mathrm{N}}=0.9$ ) has been compared with the Cordey scaling. Despite possible concerns due to lower JET-ILW pedestal performance, the agreement between the two scalings are rather reasonable for the exponents of $I_{p}$ and triangularity. However, the JET-ILW scaling shows a weaker exponent for the power and a stronger exponent for the isotope mass. Moreover, the effect of the inclusion of the $n_{e}{ }^{\text {sep }}$ $/ n_{e}^{\text {ped }}$ term in the scaling law has been tested. The inclusion of this term has slightly improved the pedestal predictions, but only marginally. Finally, a scaling for the pedestal density and temperature has been provided. The pedestal density scaling produces rather reliable results, which are in agreement with the conclusions achieved in section 5 looking at specific datasets. On the hand, the scaling for the pedestal temperature has a rather low $\mathrm{R}^{2}$ and its possible use for $T_{e}^{\text {ped }}$ prediction should be considered with extreme care. For example, while the increase of $T_{e}^{p e d}$ with $I_{p}$ has been observed for specific subsets, see figure 7(b), the scaling law shows no correlation between $T_{e}^{\text {ped }}$ with $I_{p}$.

The work has highlighted several empirical trends. Even if most of them are reasonable and have been observed in other machines and/or theoretically understood in L-mode plasmas, they still lack a full theoretical explanation. The following is a list of the open issues that needs further investigation.

(i) The increase of the pedestal width with power in the non-PB limited pedestal. In PB limited pedestal, this behavior is consistent with the EPED1 theoretical framework.

(ii) The link between divertor configuration, neutral pressure and separatrix density. Despite this has been seen in other machines and theoretical modelled in JET-ILW Lmode plasmas, no modelling has been done yet in JET-ILW H-mode plasmas.

(iii) The link correlation between $\alpha_{c r i t} / \alpha_{\text {exp }}$ and the relative shift. So far, this remain an empirical correlation. Some possible mechanisms have been discussed in the paper, but the clear physics reasons why the pedestal gradient is lower than the PB predictions and what triggers the ELM are still elusive. 


\section{APPENDIX 1}

Figure 34 shows the comparison of the key pedestal parameters determined using the mtanh fit and the linear fit described in sections 2 and 3. In general, the two fits leads to very similar pedestal parameters when the core slope is rather low. Here, for core slope is meant the slope inside the pedestal top at $\psi_{\mathrm{N}}<0.9$, see figure 1. For the temperature, the core slope can be rather high, so the difference between the two definitions can be significant. This is shown in figure 34(a), where the ratio between the $T_{e}^{\text {ped }}$ determined with the linear and mtanh fit is shown. When the core slope is high, the difference can be around $20 \%$ and up to $30 \%$ in some extreme cases. On the other hand, the difference is negligible for $n_{e}{ }^{p e d}$, see figure 34(b).

The comparison of the pedestal width is shown in figure 34(c) and 34(d). On average, the pedestal width determined with the linear fit is $50 \%$ higher for the temperature and $30 \%$ for the density.

The comparison of the pedestal position is shown in figure 34(e) and 34(f). On average, the pedestal position determined with the linear fit is $6 \%$ lower for the temperature and $3 \%$ for the density.

Figure 35 shows the comparison of the three definitions of pedestal pressure width. The mtanh fitting function has been used. Figure 35(a) shows the ratio between the standard definition used so far in JET $\left(w_{p e}^{E P E D}\right)$ and the pressure width determined by fitting the product of the deconvolved fits $\left(w_{p e}\right)$. The standard definition is approximately $20 \%-30 \%$ higher. Figure 35(b) shows the ratio between the width determined till the LCFS $\left(w_{p e}^{E P E D \psi_{N}=1}\right)$ and $w_{p e}$. A systematic difference related to the position of the pedestal density is present. This is because part of the density tends to be outside the separatrix, so the definition $w_{p e}^{E P E D \psi_{N}=1}$ produces a lower width when the density moves outwards. Note that the three definitions leads to qualitatively similar conclusions, as shown in figure 13

It should be highlighted that the differences highlighted in figures 34 and 35 are only matter of definitions and do not affect the correlations discussed in this work. Moreover, the linear fit has a limited use, as it cannot be employed for the PB stability analysis due to the discontinuity in the radial derivative. For this reason, the mtanh fit is used throughout this work. The inclusion of the linear fit in the database is motivated by a possible need of comparison with earlier results from other machines.

Figure 36 shows the comparison between the experimental $\mathrm{W}_{90}$ and the pedestal stored energy determined with the Cordey scaling for a set of JET-C pulses. The JET-C pulses have been selected randomly in order to include plasmas with a range of engineering parameters as wide 
as possible. The figure shows that $\mathrm{W}_{90}$ is a good proxy to be used for comparison with the Cordey scaling.
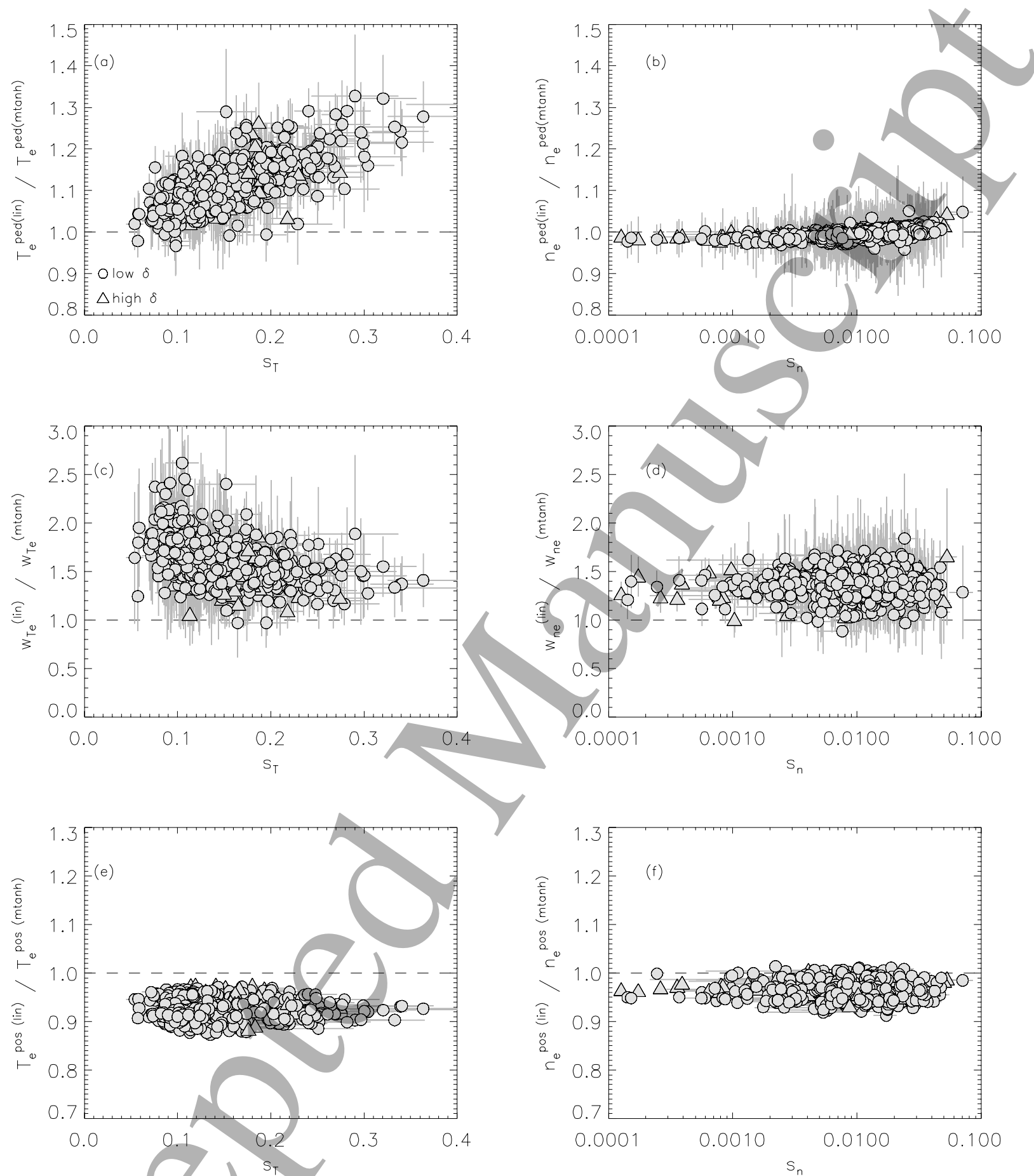

Figure 34. Comparison of the pedestal parameters determined using the linear fit and the mtanh fit. The figure shows the ratio between the parameter determined with the linear and the mtanh fit for (a) temperature height, (b) density height, (c) temperature width, (d) density width, (e) temperature position and (f) density position. The horizontal axis is the parameter corresponding to the core slope in the mtanh definition, see expression (2). 

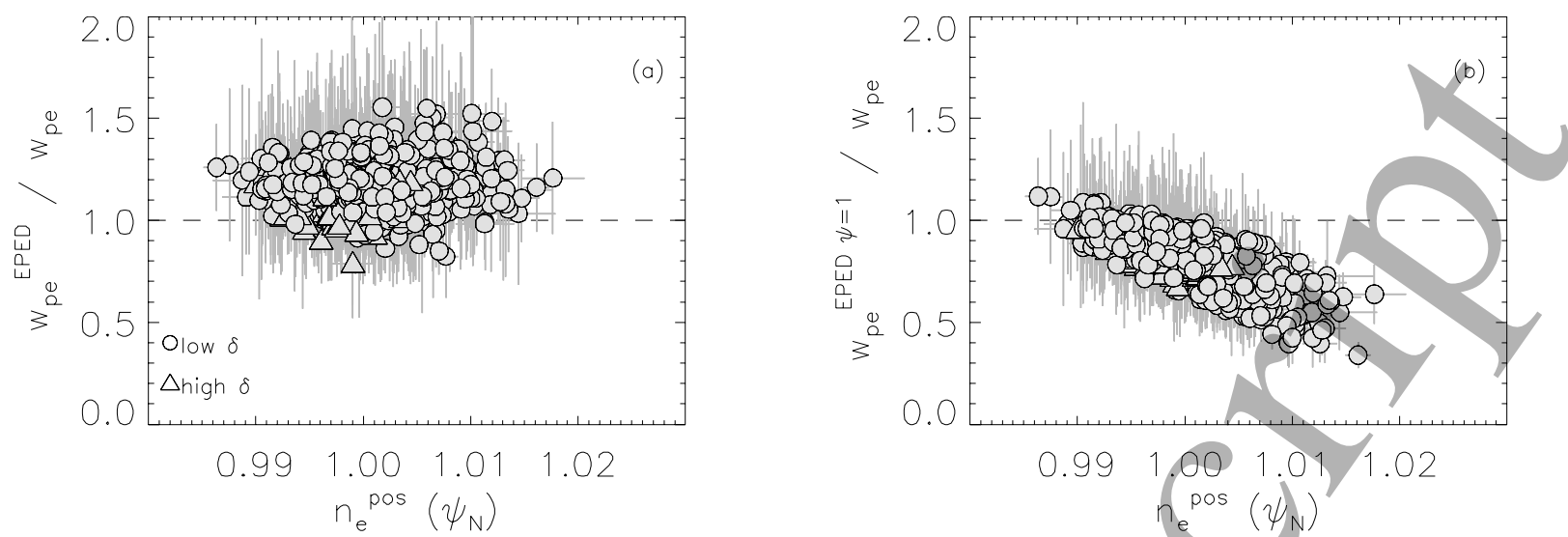

Figure 35. Comparison of the pedestal pressure width definitions. (a) ratio between the standard JET definition $\left(w_{p e}^{E P E D}\right)$ and the width determined by fitting the deconvolved fits (wpe). (b) Ratio between the standard width determined till LCFS and wpe. The horizontal axis is the position of the pedestal density. The mtanh fitting function has been used.

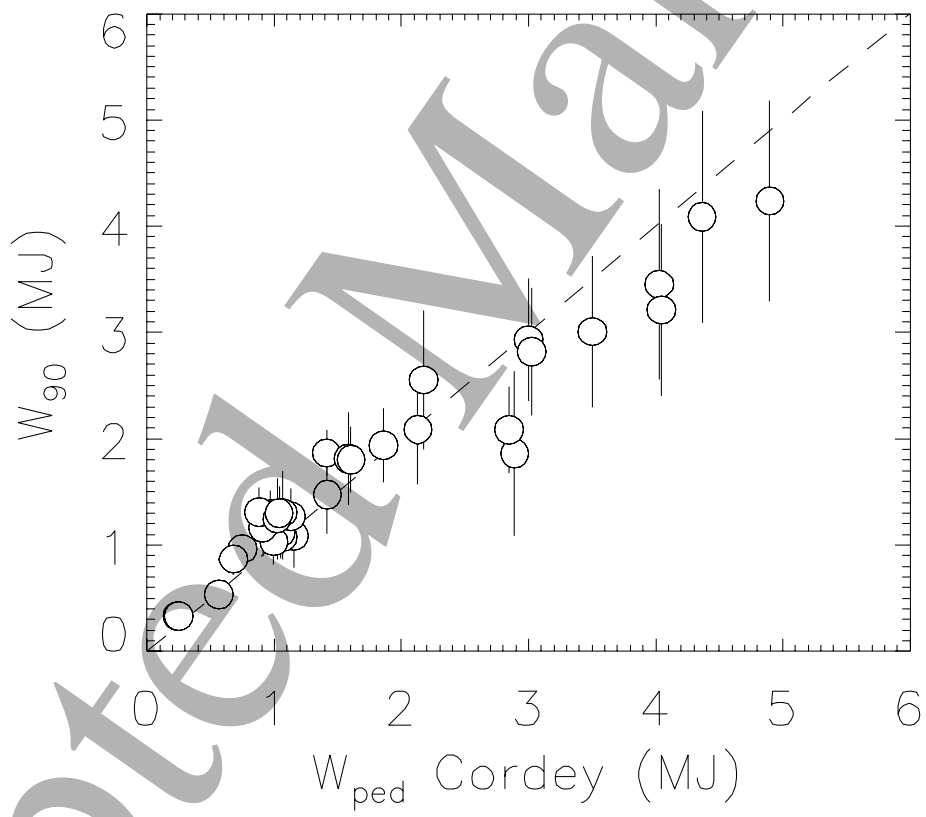

Figure 36. Comparison between the experimental $W_{90}$ determined for a set of JET-C pulses and the corresponding pedestal stored energy determined using the Cordey scaling. 


\section{APPENDIX 2}

Figure 12(b) and figure 22(d) have shown that at high relative shift (approximately $n_{e}{ }^{\text {pos }}$ $\left.T_{e}{ }^{p o s}>0.02 \psi_{\mathrm{N}}\right)$, a change in the density position does not affect the PB stability. As stated in section 5 , this is because at high relative shift the density position has a minor effect of the pressure position. This claim was initially verified in reference [39]. This Appendix and figure 37 describe in details the geometrical interpretation of this effect.
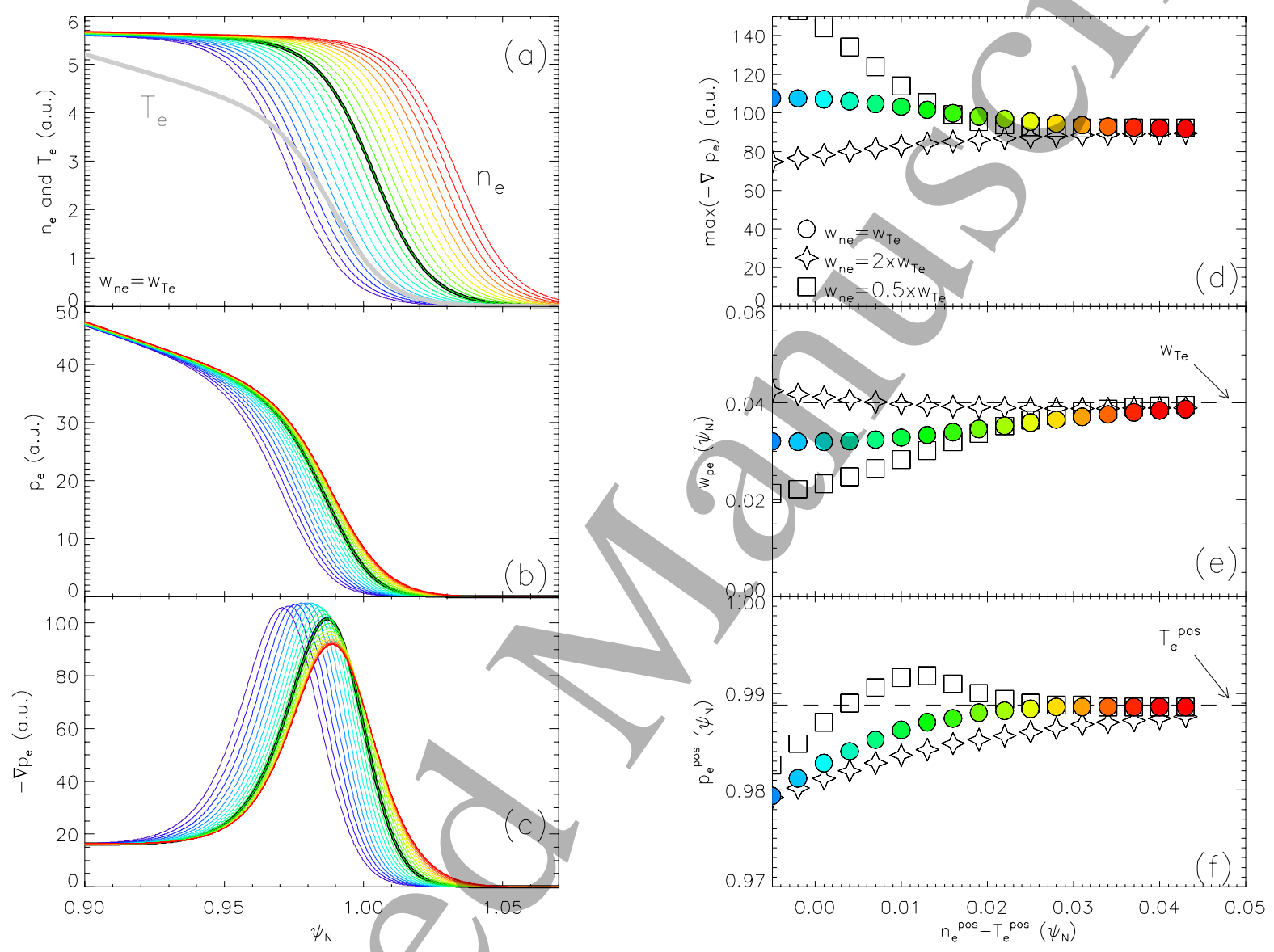

Figure 37.(a) The thick lines shows the shape of typical $T_{e}$ and $n_{e}$ profiles in JET-ILW assuming $w_{T e}=w_{n e}$. The colored profiles show shifted $n_{e}$ profiles. (b) Corresponding shapes of the pressure profiles and (c) their gradients. Corresponding (d) maximum pressure gradients, (e) pressure widths and (f) pressure positions versus the relative shift. The circles represent the case of frames (a)-(c) assuming $w_{T e}=w_{n e}$. The stars and the square show two opposite extreme cases, with $w_{n e}=2 w_{T e}$ and $w_{n e}=0.5 w_{T e}$. The experimental range of JET-ILW corresponds to $n_{e}^{\text {pos }}-T_{e}^{\text {pos }}>0.01 \psi_{\mathrm{N}}$.

The thick lines in figure 37(a) show the shape of typical $T_{e}$ and $n_{e}$ profiles in JET-ILW (in arbitrary units since only the qualitative trends of pressure shape will be discussed). For 
simplicity, $w_{T e}=w_{n e}$ has been assumed. From the product of the $T_{e}$ and $n_{e}$ profiles it is possible to calculate the $p_{e}$ profile and the corresponding pressure pedestal gradient, width and position (the width is determined with a mtanh fit). Then, starting from this reference case, the procedure has been repeated by shifting outwards and inwards the density profile, as shown by the colored profiles in figure 37(a). The corresponding pressure and pressure gradient profiles are shown in figures 37(b) and 37(c). The maximum pressure gradients, the pressure pedestal widths and the pressure pedestal positions versus the relative shift are shown in figures $37(\mathrm{~d}), 37(\mathrm{e})$ and $37(\mathrm{f})$, respectively. Note that the experimental range of JET-ILW corresponds to $n_{e}^{p o s}-T_{e}^{p o s}>0.01 \psi_{\mathrm{N}}$. The effect of the density shift on the maximum pressure gradient is minimal. The outward shift of the density leads to a small widening of the pedestal width and to an outward shift of the pedestal pressure. However, these effects clearly saturate for $n_{e}$ pos $-T_{e}^{\text {pos }}>0.02 \psi_{\mathrm{N}}$. This is because, when the density is too much outwards, the maximum density gradient is located in the region where $T_{e} \approx 0$. So, the shape of the pressure profile is determined mainly by the temperature profile. In fact, for $n_{e}^{p o s}-T_{e}^{p o s}>0.02 \psi_{\mathrm{N}}, w_{p e}$ and $p_{e}^{p o s}$ converge to the values of $w_{T e}$ and $T_{e}^{p o s}$ (see the dashed lines in figures $37(\mathrm{e})$ and 37(f)). The qualitative trends shown in figure 37 might depend also on the pedestal width. Therefore, the procedure has been repeated for two extreme cases, assuming $w_{n e}=2 w_{T e}$ (see the star symbols in figure 37) and assuming $w_{n e}=0.5 w_{T e}$ (square symbols). The qualitative trends of these two cases are different from the reference case $w_{n e}=w_{T e}$ only for very small relative shift, while they are similar to the reference case in the experimental range of JET-ILW relative shifts $\left(n_{e}^{p o s}-T_{e}^{p o s}>0.01 \psi_{\mathrm{N}}\right)$. In particular, at high relative shift all the three cases show a minimal effect of $n_{e}{ }^{\text {pos }}$ on pressure width and position converging to the values of $w_{T e}$ and $T_{e}^{\text {pos }}$. 


\section{ACKNOWLEDGEMENTS}

This research is supported by Vetenskapsrådet, under grant number 2019-04618.

This work has been carried out within the framework of the EUROfusion Consortium and has received funding from the Euratom research and training programme 2014-2018 and 2019-2020 under grant agreement No 633053. The views and opinions expressed herein do not necessarily reflect those of the European Commission.

The scientific work is published for the realization of the international project co-financed by Polish Ministry of Science and Higher Education in 2019 from financial resources of the program entitled 'PMW'; Agreement No. 5040/H2020/Euratom/2019/2"

\section{REFERENCES}

[1] Christiansen J.P. et al., Nucl. Fusion 32 (1992) 291.

[2] Thomsen K. et al., Nucl. Fusion 34 (1994) 131.

[3] Hatae T. et al., Nucl. Fusion 41 (2001) 285.

[4] Kardoun J.W.F et al., "Next step Tokamak physics: confinement-oriented global database analysis", 2000 IAEA Fusion Energy Conference, Sorrento, ITERP/04.

[5] Thomsen K. et al., Plasma Phys. Controll. Fusion 44 (2002) A429.

[6] Cordey J.G. et al., "A two term model of the confinement in ELMy H-modes using the global confinement and pedestal databases". 2002 IAEA Fusion Energy Conference, Lyon, CT/P-02.

[7] Cordey J. G. et al., Nucl. Fusion 43 (2003) 670.

[8] Horton L. et al., Plasma Phys. Controll. Fusion 44 (2002) A273.

[9] Mc Donald D.C. et al., Nucl. Fusion 47 (2007) 174.

[10] Garcia J. et al., Plasma Phys. Control. Fusion 59, 014023 (2017)

[11] Garcia J. et al., "First principles and integrated modelling achievements towards trustful Fusion power predictions for JET and ITER". Preprint: 2018 IAEA Fusion Energy Conference, Gandhinagar, TH/3-1.

[12] Casson F. et al., "Predictive multi-channel flux-driven modelling to optimise ICRH tungsten control in JET". Preprint: 2018 IAEA Fusion Energy Conference, Gandhinagar, $\mathrm{TH} / 3-2 \mathrm{Ra}$.

[13] Garcia J. et al., Nucl. Fusion 59, 086047 (2019)

[14] Casson F. et al., Nucl. Fusion 60, 066029 (2020)

[15] S. Brezinszek et al., ”24th IAEA Fusion Energy Conference”, San Diego, USA, 2012, pp EX/4-1.

[16] G. Matthews et al., J. Nucl. Mat. 438, S2 (2013)

[17] Giroud C.et al., Nucl. Fusion 53113025 (2013)

[18] Beurskens M et al., Nucl. Fusion 54, 043001 (2014)

[19] Nunes I, Plasma Physics and Control. Fusion 58, 014034 (2016)

[20] Maggi C et al., Plasma Physics and Control. Fusion 60, 014045 (2018)

[21] ITER Physics Basis 1999 Nucl. Fusion 39, 2175 (1999)

[22] Cordey J.G. et al., Plasma Phys. Control. Fusion 38, A67 (1996)

[23] Hughes J.W. et al., Nucl. Fusion 51, 083007 (2011)

[24] Giroud C.et al., Nucl. Fusion 52063022 (2012)

[25] Leyland M. et al. Nucl. Fusion 53, 083028 (2013)

[26] Giroud C.et al., Plasma Phys. Control. Fusion 57035004 (2015) 
[27] Maggi C. et al., Nucl. Fusion 55, 113031 (2015)

[28] Dunne M. et al., Plasma Phys. Control. Fusion 59, 014017 (2017)

[29] Sheikh U. et al., Plasma Phys. Control. Fusion 61, 014002 (2019)

[30] de la Luna E. et al., "Recent Results on High-Triangularity H-mode Studies in JET-ILW" 2016 IAEA Fusion Energy Conference, Kyoto. EX/P6-11.

[31] Joffrin E. et al., Nucl. Fusion 57086025 (2017)

[32] Giroud C. et al., "Optimisation of JET-DT and ITER operation by developing an understanding of the role of low-Z impurity on the H-mode pedestal". Preprint: 2018 IAEA Fusion Energy Conference, Gandhinagar, EX/3-3.

[33] Reinke M.L. et al., J. Nucl. Material 415, S340 (2011).

[34] Beurskens M. et al., Plasma Phys. Control. Fusion 55, 124043 (2013)

[35] P.A. Schneider et al., Plasma Phys. Control. Fusion 57, 014029 (2015)

[36] Beurskens M. et al., Nucl. Fusion 56, 056014 (2016)

[37] Dunne M. et al., Plasma Phys. Control. Fusion 59, 025010 (2017)

[38] Dunne M. et al., "Impact of impurity seeding on pedestal structure in ASDEX Upgrade and Alcator C-Mod”. Preprint: 2018 IAEA Fusion Energy Conference, Gandhinagar, $\mathrm{EX} / \mathrm{P} 8-2$.

[39] Frassinetti L. et al., Nucl. Fusion 59, 076038 (2019)

[40] Zohm H et al., Plasma Phys. Control. Fusion 38, 105 (1996)

[41] Loarte A. et al., Plasma Phys. Control. Fusion 44, 1815 (2002)

[42] Loarte A. et al., Plasma Phys. Control. Fusion 45, 11549 (2003)

[43] Saibene G. et al., Plasma Phys. Control. Fusion 44, 1769 (2002)

[44] Perez C.P. et al., Plasma Phys. Control. Fusion 50, 065018 (2008)

[45] Sartori R. et al., Plasma Phys. Control. Fusion 46, 723 (2004)

[46] Wilson H.R. et al., Phys. Plasmas 91277 (2002)

[47] Snyder P.B. et al., Phys. Plasmas 92037 (2002)

[48] Kirk A. et al, Plasma Phys. Control. Fusion 51065016 (2009)

[49] Urano H., Nucl. Fusion 54, 116001 (2011)

[50] J.R. Walk et al. , Nucl. Fusion 52063011 (2012)

[51] Groebner R. et al., Nucl. Fusion 53, 093024 (2013)

[52] Beurskens M. et al., Nucl. Fusion 53013001 (2013)

[53] Frassinetti L. et al., Nucl. Fusion 57, 061012 (2017)

[54] Frassinetti L. et al., Plasma Phys. Control. Fusion 59, 014014 (2017)

[55] Aiba N. et al., Nucl. Fusion 57, 126001 (2017)

[56] Aiba N. et al., Nucl. Fusion 60, 014032 (2018)

[57] Snyder P.B. et al., Phys. Plasmas 16056118 (2009)

[58] Snyder P.B. et al., Nucl. Fusion 51103016 (2011)

[59] Maggi C. et al., Nucl. Fusion 57, 116012 (2017)

[60] https://users.euro-fusion.org/

[61] A. Diallo et al., Nucl. Fusion 53, 093026 (2013)

[62] P.A. Schneider et al., Plasma Phys. Control. Fusion 54, 105009 (2012)

[63] H. Urano et al., Nucl. Fusion 48, 045008 (2008)

[64] H. Urano et al., Nucl. Fusion 49, 095006 (2009)

[65] H. Urano et al., Nucl. Fusion 56016005 (2016)

[66] Saarelma S. et al., Phys. Plasmas 26, 072501 (2019)

[67] Mahdavi M. A. et al., Physics of Plasmas 10, 3984 (2003)

[68] Willensdorfer M. et al., Nucl. Fusion 53, 093020 (2013)

[69] Leonard A. et al, Jour. Nuc. Mater. S246 (2013)

[70] Groebner R.J et al., Phys. Plasmas 9, 2134 (2002).

[71] Mordijck et al., Nucl. Fusion 60, 082006 (2020)

[72] Stefanikova E. et al., Nucl Fusion 58, 056010 (2018). 
[73] Hatch D.R. et al., Nucl Fusion 57, 036020 (2017).

[74] Hatch D.R. et al., Nucl Fusion 56, 104003 (2016).

[75] Hatch D.R. et al., Nucl. Fusion 59, 086056 (2019).

[76] M. Kotschenreuther et al., Nucl. Fusion 59096001 (2019)

[77] Pasqualotto R., et al., Rev. Sci. Instrum. 75, 3891 (2004).

[78] Stangeby P.C. 2000 The Plasma Boundary of Magnetic Fusion Devices (Bristol: Institute of Physics Publishing)

[79] J. Simpson et al., Nuclear Materials and Energy 20100599 (2019).

[80] Frassinetti L. et al., Nucl. Fusion 55023007 (2015)

[81] Frassinetti L., et al., Rev. Sci. Instrum. 83, 013506 (2012)

[82] Groebner R.J. et al., Nucl. Fusion 411789 (2001)

[83] Perez C.P. et al., Nucl. Fusion 59, 056004 (2019)

[84] Miller R.L. Phys. Plasmas 5973 (1998)

[85] Huysmans G.T.A. et al 1991 Computational Physics: Proc. Int. Conf. (Amsterdam, The Netherlands) (Singapore: World Scientific)

[86] Sauter O. and Angioni C. Phys. Plasmas 62834 (1999)

[87] Hager R. Phys. Plasmas 23, 042503 (2016)

[88] Horvath L. Plasma Phys. Control. Fusion 60, 085003 (2018)

[89] Belli E. et al., Plasma Phys. Control. Fusion 54 015015(2012)

[90] Belli E. et al., Plasma Phys. Control. Fusion 56045006 (2014)

[91] Saarelma S. et al., Phys. Plasmas 22056115 (2015)

[92] Imbeaux F. et al., Nucl. Fusion 55 (2015) 123006.

[93] Lang P. et al., Plasma Phys. Control. Fusion 57045011 (2015)

[94] Evans T.E. et al, Nature Phys. 2419 (2006)

[95] Liang Y. et al., Nucl. Fusion 53, 073036 (2013)

[96] de la Luna E. et al. Nucl. Fusion 56, 026001 (2015)

[97] Artola F.J. et al. Nucl. Fusion 58, 096018 (2018)

[98] Maggi C. et al., Nucl. Fusion 59,076028 (2019)

[99] Horvath L. et al., "Isotope dependence of the pedestal in JET-ILW type I ELMy H-modes", 46th EPS Conf. on Plasma Physics, Milano, Italy, 8-12 July 2019, O4.102

[100] King D.B. et al., "Mixed Hydrogen-Deuterium plasmas on JET ILW: H-mode confinement and isotope mixture control" 44th EPS Conf. on Plasma Physics, Belfast, Northern Ireland, 26-30 June 2017, O3.112

[101] Challis C., et al., Nucl. Fusion 55, 053031 (2018)

[102] Beurskens M.N.A. et al., Plasma Phys. Control. Fusion 51, 124051 (2009)

[103] Beurskens M.N.A. et al., Phys. Plasmas 18, 056120 (2011)

[104] Lönnroth J. et al., Nucl. Fusion 51, 013003 (2011)

[105] Kirk A. et al., Nucl. Fusion 55043011 (2015)

[106] W. Obert, et al., Performance of the JET Pumped Divertor Cryopumpsystem, 16th IEEE/NPSS

Symposium (1995) 742-745, http://dx.doi.org/10.1109/FUSION.1995.534329.

[107] Moulton D. et al., "Pumping in vertical and horizontal target configurations on JET in Lmode; an interpretive study using EDGE2D-EIRENE", 42th EPS Conf. on Plasma Physics, Lisbon, Portugal, 22-26 June 2015, O4.119

[108] Uljanoys J et al., Nuclear Materials and Energy 12, 791-797 (2017)

[109] Martin Y. et al., J. Phys. Conf. Ser. 123, 012033 (2008)

[110] Garzotti L. et al., Nucl. Fusion 59, 076037 (2019)

[111] Joffrin L. et al., Nucl. Fusion 59, 112021 (2019)

[112] Kallenbach A., Nuclear Materials and Energy 18, 166 (2019)

[113] Kallenbach A., Plasma Phys. Control. Fusion 60, 045006 (2018)

[114] Wang H.Q et al., Nucl. Fusion 58, 096014 (2018)

[115] Moser A.L. et al., Phys. Plasmas 27, 032506 (2020) 
[116] Sontag A.C. et al 2017 Nucl. Fusion 57076025

[117] Kruezi U. et al., Rev. Sci. Instr. 83, 10D728 (2012)

[118] Groth M. et al., Nucl. Fusion 53093016 (2013)

[119] Groth M. et al., Journal of Nuclear Materials 463, 471 (2015)

[120] Pitcher C and Stangeby P, Plasma Phys. Control. Fusion 39, 779 (1997)

[121] Wiesen S. et al., Nuclear Materials and Energy 12, 3-17 (2017)

[122] Varoutis S. et al., Fus. Eng. Des 121, 13 (2017)

[123] S. Saarelma et al., Plasma Phys. Control. Fusion 60, 014042 (2018)

[124] R. Maingi, Phys. Rev. Lett. 103, 075001 (2009)

[125] R. Maingi et al., Nucl. Fusion 52083001 (2012)

[126] T.H. Osborne et al., Nucl. Fusion 55063018 (2015)

[127] Reimold et al., Nucl. Mater. Energy 12, 193 (2017)

[128] Urano H. et al., Nucl. Fusion 46781 (2006)

[129] Vermare Nucl. Fusion 47490 (2007).

[130] R.J. Groebner et al., Nucl. Fusion 49085037 (2009)

[131] Stober J. et al., Plasma Phys. Control. Fusion 42, A211 (2000)

[132] Urano H, Nucl. Fusion 54116001 (2014)

[133] Hobirk J. et al., Plasma Phys. Control. Fusion 54, 095001 (2012)

[134] Sips A.C.C. et al., Nucl. Fusion 58126010 (2018)

[135] Snyder P.B. et al., Plasma Phys. Controll. Fusion 46 A131 (2004)

[136] Huysmans G et al., Nucl. Fusion 47659 (2007)

[137] Pamela S. et al., Nucl. Fusion 57076006 (2017)

[138] Wu N. et al., Phys. Plasmas 2509305 (2018)

[139] Parisi et al., submitted to Nucl. Fusion

[140] M. Kotschenreuther et al.,Comput.Phys.Comm. 88, 128 (1995)

[141] Urano H., Proc. 43rd EPS Conference on Plasma Physics, 4 - 8 July, 2016, Leuven, Belgium, O4.121

[142] Verdoolaege G. et al., “ Regression analysis in the updated ITPA global H-mode confinement database using robust Bayesian and minimum distance estimation methods", $3{ }^{\text {rd }}$ IAEA Technical Meeting on Fusion Data Processing, Validation and Analysis, Vienna, 27-31 May 2019.

[143] Verdoolaege G., Entropy 17, 4602 (2015).

[144] Maslov M. et al., Nucl. Fusion 60, 036007 (2020).

[145] Maggi C.M. et al., "Isotopic effect on particle and heat transport", 17th International Workshop on H-mode Physics and Transport Barriers, 911 October 2019, Shanghai (China).

[146] Hatch D. et al., private communication.

[147] Chen X. et al., Nucl. Fusion 57, 022007 (2017). 